\title{
Mueller Navelet jets at LHC - complete next-to-leading BFKL calculation
}

\author{
Dimitri Colferai, ${ }^{a, b}$ Florian Schwennsen, ${ }^{c}$ Lech Szymanowski ${ }^{d, e}$ and Samuel Wallon ${ }^{f, g}$ \\ ${ }^{a}$ Dipartimento di Fisica, Università di Firenze, \\ Via G. Sansone 1, 50019 Firenze, Italy \\ ${ }^{b}$ INFN, Sezione di Firenze, \\ Via G. Sansone 1, 50019 Firenze, Italy \\ ${ }^{c}$ Deusches Elektronen-Synchrotron DESY, \\ Notkestrasse 85, 22603 Hamburg, Germany \\ ${ }^{d}$ Soltan Institute for Nuclear Studies, \\ ul. Hoza 69, 00-681 Warsaw, Poland \\ ${ }^{e}$ CPHT, École Polytechnique, CNRS, \\ 48 Route de Saclay, 91128 Palaiseau Cedex, France \\ ${ }^{f}$ LPT, Université Paris-Sud, CNRS, \\ Batiment 210, 91405 Orsay, France \\ ${ }^{g}$ UPMC Univ. Paris 06, faculté de physique, \\ 4 place Jussieu, 75252 Paris Cedex 05, France \\ E-mail: colferai@fi.infn.it, florian.schwennsen@desy.de, \\ lech.szymanowski@fuw.edu.pl, samuel.wallon@th.u-psud.fr
}

ABSTRACT: We calculate cross section and azimuthal decorrellation of Mueller Navelet jets at the LHC in the complete next-lo-leading order BFKL framework, i.e. including nextto-leading corrections to the Green's function as well as next-to-leading corrections to the Mueller Navelet vertices. The obtained results for standard observables proposed for studies of Mueller Navelet jets show that both sources of corrections are of equal, big importance for final magnitude and final behavior of observables. The astonishing conclusion of our analysis is that the observables obtained within the complete next-lo-leading order BFKL framework of the present paper are quite similar to the same observables obtained within next-to-leading DGLAP type treatment. This fact sheds doubts on general belief that the studies of Mueller Navelet jets at the LHC will lead to clear discrimination between the BFKL and the DGLAP dynamics.

Keywords: Jets, NLO Computations, QCD, Hadronic Colliders

ArXiv ePrint: 1002.1365 


\section{Contents}

1 Introduction $\quad 1$

2 LL calculation $\quad 3$

2.1 Kinematics 3

2.2 LL BFKL calculation 3

3 NLL calculation $\quad 6$

3.1 Strong coupling, renormalization scheme and PDFs at NLL 7

$\begin{array}{lll}3.2 & \text { Jet vertices at NLL } & 7\end{array}$

$\begin{array}{lll}3.2 .1 & \text { Jet definition } & 9\end{array}$

$\begin{array}{lll}3.2 .2 & \text { LL subtraction and } s_{0} & 10\end{array}$

$\begin{array}{lll}3.3 & \text { BFKL Green's function at NLL } & 11\end{array}$

$\begin{array}{lll}\text { 3.3.1 Collinear improved Green's function } & 12\end{array}$

3.3.2 Approximate energy-momentum conservation in BFKL 13

4 Results 13

$4.1\left|\mathbf{k}_{J, 1}\right|=\left|\mathbf{k}_{J, 2}\right|=35 \mathrm{GeV} \quad 14$

$4.2\left|\mathbf{k}_{J, 1}\right|=\left|\mathbf{k}_{J, 2}\right|=50 \mathrm{GeV}$

$4.3\left|\mathbf{k}_{J, 1}\right|=35 \mathrm{GeV},\left|\mathbf{k}_{J, 2}\right|=50 \mathrm{GeV} \quad 26$

5 Conclusions $\quad 35$

$\begin{array}{lr}\text { A Details on the numerical implementation } & 36\end{array}$

A.1 Programs used 36

$\begin{array}{lll}\text { A.2 Choice of parameters } & 36\end{array}$

$\begin{array}{lll}\text { A.2.1 The } \nu \text {-grid } & 36\end{array}$

$\begin{array}{lll}\text { A.3 Grouping the integrand } & 37\end{array}$

$\begin{array}{ll}\text { B Tabled values of diagrams } & 39\end{array}$

\section{Introduction}

The large center of mass energy of hadron colliders like the Tevatron and the Large Hadron Collider (LHC) is not only of interest for the production of possible new heavy particles, but also allows to investigate the high energy regime of Quantum Chromodynamics (QCD). An especially interesting situation appears if two different large scales enter the game. If the two scales are ordered, large logarithms of the ratio of the two scales compensate the smallness of the coupling and therefore have to be resummed to all orders. One famous example is the case of high energy scattering with fixed momentum transfer. If the center 


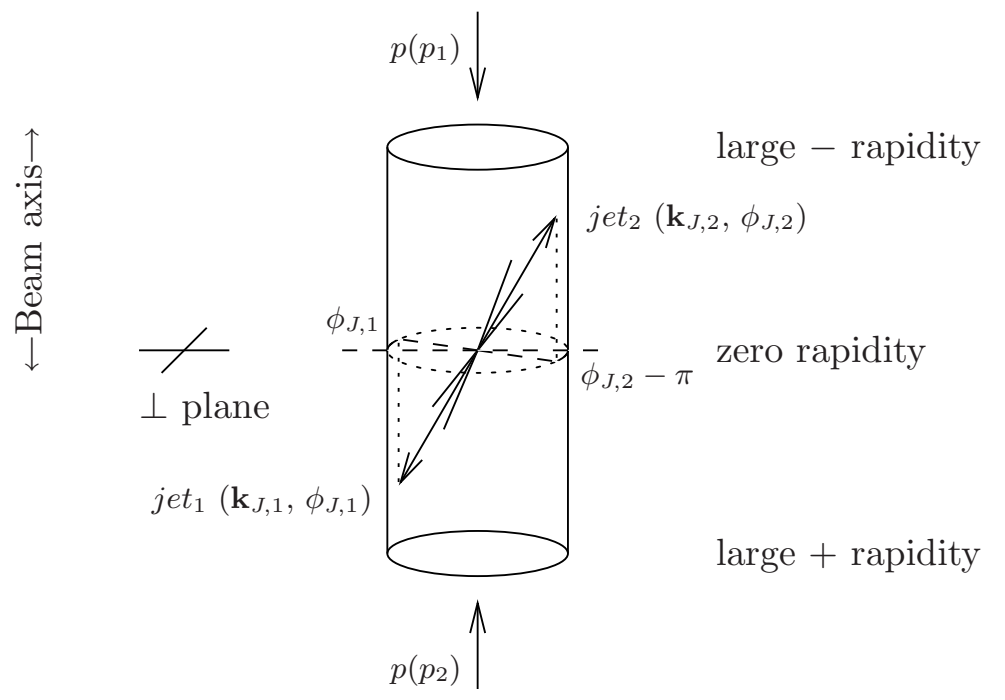

Figure 1. Mueller Navelet jets, illustrated at lowest order.

of mass energy $s$ is much larger than the momentum transfer $|t|$ - the so-called Regge asymptotics of the process - the gluon exchange in the crossed channel dominates and logarithms of the type $\left[\alpha_{s} \ln (s /|t|)\right]^{n}$ have to be resummed. This is realized by the leading logarithmic (LL) Balitsky-Fadin-Kuraev-Lipatov (BFKL) [1-4] equation for the gluon Green's function describing the momentum exchange in the $t$-channel.

One of the most famous testing ground for BFKL physics are the Mueller Navelet jets [5], illustrated in figure 1 . The predicted power like rise of the cross section with increasing energy has been observed at the Tevatron $p \bar{p}$-collider [6], but the measurements revealed an even stronger rise than predicted by BFKL calculations. Beside the cross section also a more exclusive observable within this process drew the attention, namely the azimuthal correlation between these jets. Considering hadron-hadron scattering in the common parton model to describe two jet production at LO, one deals with a back-to-back reaction and expects the azimuthal angles of the two jets always to be $\pi$ and hence completely correlated. This corresponds in figure 1 to $\phi_{J, 1}=\phi_{J, 2}-\pi$. But when we increase the rapidity difference between these jets, the phase space allows for more and more emissions leading to an angular decorrelation between the jets. In the academical limit of infinite rapidity, the angles should be completely uncorrelated. In the regime of large, but realizable rapidity differences the resummation of large logarithms calls for a description within the BFKL theory. Unfortunately, the leading logarithmic approximation $[7,8]$ overestimates this decorrelation by far. Improvements have been obtained by taking into account some corrections of higher order like the running of the coupling $[9,10]$. In particular, the effect of energy-momentum conservation, which is beyond BFKL approximation, was shown to have an important impact for large rapidity separation of jets [9]. In our present study this can affect the reliability of the predictions at the borders of the phase space, as discussed in section 3.3.2. Some earlier calculations with the next-to-leading (NLL) BFKL Green's function have been published in ref. [11, 12]. 
In this paper we present the full NLL BFKL calculation where also the NLL result for the Mueller Navelet vertices $[13,14]$ will be taken into account. In section 2 we recall the LL BFKL calculation deriving also the key formulas which are then used in section 3 where the NLL calculation is presented. In section 4 we present results and give a summary in section 5. Technical details of the numerical implementation are given in an appendix.

\section{LL calculation}

\section{$2.1 \quad$ Kinematics}

The kinematic setup is schematically shown in figure 2 . The two hadrons collide at a center of mass energy $s$ producing two very forward jets, the transverse momenta of the jets are labeled by Euclidean two dimensional vectors $\mathbf{k}_{J, 1}$ and $\mathbf{k}_{J, 2}$, while their azimuthal angles are noted as $\phi_{J, 1}$ and $\phi_{J, 2}$. We will denote the rapidities of the jets by $y_{J, 1}$ and $y_{J, 2}$ which are related to the longitudinal momentum fractions of the jets via $x_{J}=\frac{\left|\mathbf{k}_{J}\right|}{\sqrt{s}} e^{y_{J}}$.

At any real experiment transverse momenta as well as rapidities are measured within certain intervals. A proper theoretical calculation should take this into account and integrate $\left|\mathbf{k}_{J, i}\right|$ and $y_{J, i}$ over the according interval. However, since at the LHC the binning in rapidity and in transverse momentum will be quite narrow [15], we consider the case of fixed rapidities and transverse momenta.

\subsection{LL BFKL calculation}

Due to the large longitudinal momentum fractions $x_{J, 1}$ and $x_{J, 2}$ of the forward jets, collinear factorization holds and the differential cross section can be written as

$$
\frac{\mathrm{d} \sigma}{\mathrm{d}\left|\mathbf{k}_{J, 1}\right| \mathrm{d}\left|\mathbf{k}_{J, 2}\right| \mathrm{d} y_{J, 1} \mathrm{~d} y_{J, 2}}=\sum_{\mathrm{a}, \mathrm{b}} \int_{0}^{1} \mathrm{~d} x_{1} \int_{0}^{1} \mathrm{~d} x_{2} f_{\mathrm{a}}\left(x_{1}\right) f_{\mathrm{b}}\left(x_{2}\right) \frac{\mathrm{d} \hat{\sigma}_{\mathrm{ab}}}{\mathrm{d}\left|\mathbf{k}_{J, 1}\right| \mathrm{d}\left|\mathbf{k}_{J, 2}\right| \mathrm{d} y_{J, 1} \mathrm{~d} y_{J, 2}},
$$

where $f_{\mathrm{a}, \mathrm{b}}$ are the standard parton distribution functions (PDFs) of a parton a (b) in the according proton. They depend furthermore on the renormalization scale $\mu_{R}$ and the factorization scale $\mu_{F}$.

The partonic cross section at lowest order in the collinear factorization approach would just be described by simple two-to-two scattering processes as they are discussed in standard text books. However, the necessary resummation of logarithmically enhanced contributions calls for a description of the partonic cross section in $k_{T}$-factorization:

$$
\frac{\mathrm{d} \hat{\sigma}_{\mathrm{ab}}}{\mathrm{d}\left|\mathbf{k}_{J, 1}\right| \mathrm{d}\left|\mathbf{k}_{J, 2}\right| \mathrm{d} y_{J, 1} \mathrm{~d} y_{J, 2}}=\int \mathrm{d} \phi_{J, 1} \mathrm{~d} \phi_{J, 2} \int \mathrm{d}^{2} \mathbf{k}_{1} \mathrm{~d}^{2} \mathbf{k}_{2} V_{\mathrm{a}}\left(-\mathbf{k}_{1}, x_{1}\right) G\left(\mathbf{k}_{1}, \mathbf{k}_{2}, \hat{s}\right) V_{\mathrm{b}}\left(\mathbf{k}_{2}, x_{2}\right),
$$

where $G$ is the BFKL Green's function depending on $\hat{s}=x_{1} x_{2} s$, and the jet vertex $V$ at lowest order reads $[13,14]$ :

$$
\begin{aligned}
V_{\mathrm{a}}^{(0)}(\mathbf{k}, x)=h_{\mathrm{a}}^{(0)}(\mathbf{k}) \mathcal{S}_{J}^{(2)}(\mathbf{k} ; x), & h_{\mathrm{a}}^{(0)}(\mathbf{k})=\frac{\alpha_{s}}{\sqrt{2}} \frac{C_{A / F}}{\mathbf{k}^{2}}, \\
\mathcal{S}_{J}^{(2)}(\mathbf{k} ; x) & =\delta\left(1-\frac{x_{J}}{x}\right)\left|\mathbf{k}_{J}\right| \delta^{(2)}\left(\mathbf{k}-\mathbf{k}_{J}\right) .
\end{aligned}
$$




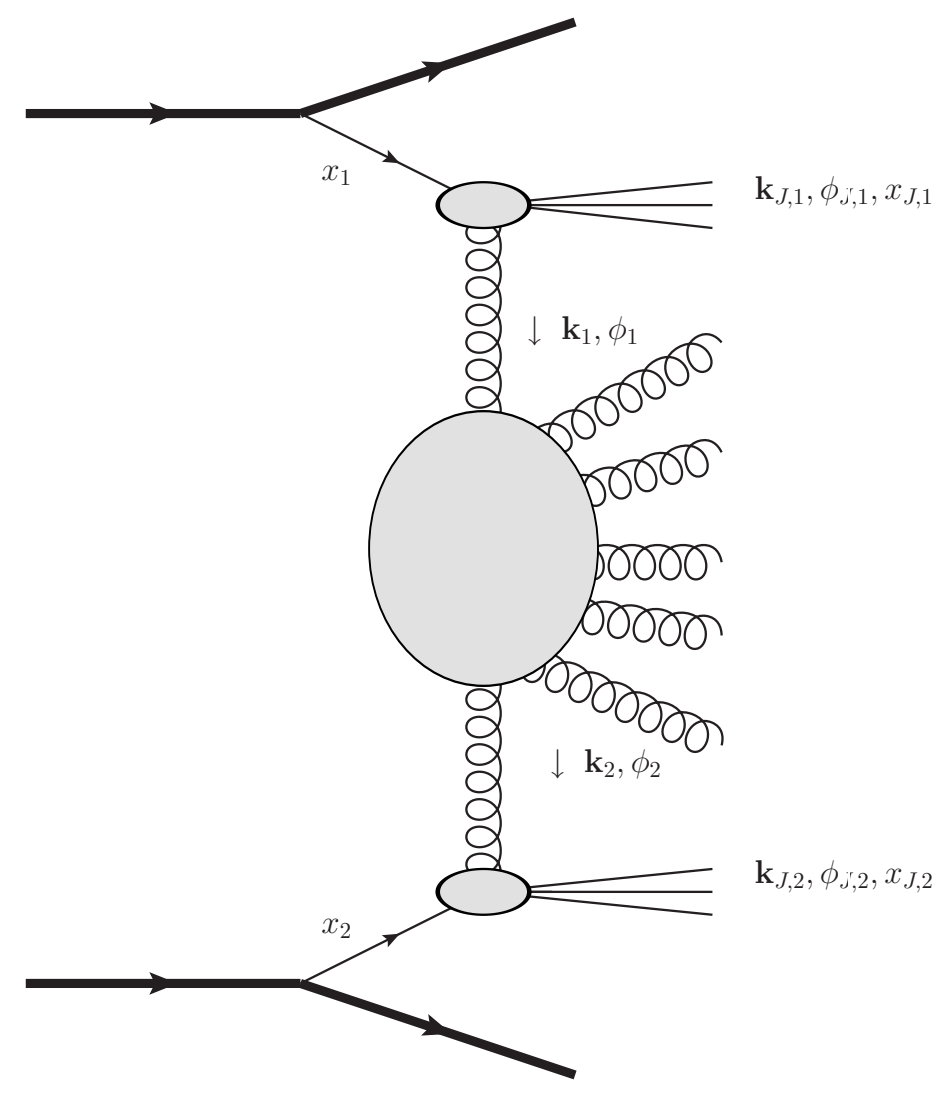

Figure 2. Schematical illustration of the kinematics as described in section 2.1.

In the definition of $h_{\mathrm{a}}^{(0)}, C_{A}=N_{c}=3$ is to be used for initial gluon and $C_{F}=\left(N_{c}^{2}-\right.$ $1) /\left(2 N_{c}\right)=4 / 3$ for initial quark. Following the notation of ref. $[13,14]$, the dependence of $V$ on the jet variables is implicit.

Combining the PDFs with the jet vertices we now write

$$
\begin{aligned}
& \frac{\mathrm{d} \sigma}{\mathrm{d}\left|\mathbf{k}_{J, 1}\right| \mathrm{d}\left|\mathbf{k}_{J, 2}\right| \mathrm{d} y_{J, 1} \mathrm{~d} y_{J, 2}}= \\
& \quad=\int \mathrm{d} \phi_{J, 1} \mathrm{~d} \phi_{J, 2} \int \mathrm{d}^{2} \mathbf{k}_{1} \mathrm{~d}^{2} \mathbf{k}_{2} \Phi\left(\mathbf{k}_{J, 1}, x_{J, 1},-\mathbf{k}_{1}\right) G\left(\mathbf{k}_{1}, \mathbf{k}_{2}, \hat{s}\right) \Phi\left(\mathbf{k}_{J, 2}, x_{J, 2}, \mathbf{k}_{2}\right),
\end{aligned}
$$

where

$$
\Phi\left(\mathbf{k}_{J, 2}, x_{J, 2}, \mathbf{k}_{2}\right)=\int \mathrm{d} x_{2} f\left(x_{2}\right) V\left(\mathbf{k}_{2}, x_{2}\right) .
$$

These $\Phi$ are no longer impact factors in the classical sense as they depend, after the convolution in $x$ with the PDF, on the total energy $s$. In the 'pure' BFKL formula of eq. (2.2) the longitudinal momentum fractions $x_{i}$ were just some external parameter and the vertices $V$ would not depend on $\hat{s}$ nor on $s$.

In view of the azimuthal decorrelation we want to investigate later, it is useful to define the following coefficients: 


$$
\begin{aligned}
& \mathcal{C}_{m} \equiv \int \mathrm{d} \phi_{J, 1} \mathrm{~d} \phi_{J, 2} \cos \left(m\left(\phi_{J, 1}-\phi_{J, 2}-\pi\right)\right) \\
& \times \int \mathrm{d}^{2} \mathbf{k}_{1} \mathrm{~d}^{2} \mathbf{k}_{2} \Phi\left(\mathbf{k}_{J, 1}, x_{J, 1},-\mathbf{k}_{1}\right) G\left(\mathbf{k}_{1}, \mathbf{k}_{2}, \hat{s}\right) \Phi\left(\mathbf{k}_{J, 2}, x_{J, 2}, \mathbf{k}_{2}\right) .
\end{aligned}
$$

Knowing these coefficients, one can easily obtain the differential cross section

$$
\frac{\mathrm{d} \sigma}{\mathrm{d}\left|\mathbf{k}_{J, 1}\right| \mathrm{d}\left|\mathbf{k}_{J, 2}\right| \mathrm{d} y_{J, 1} \mathrm{~d} y_{J, 2}}=\mathcal{C}_{0},
$$

and the following measure of azimuthal decorrelation

$$
\langle\cos (m \varphi)\rangle \equiv\left\langle\cos \left(m\left(\phi_{J, 1}-\phi_{J, 2}-\pi\right)\right)\right\rangle=\frac{\mathcal{C}_{m}}{\mathcal{C}_{0}} .
$$

By decomposing $\Phi$ in terms of the LL-BFKL eigenfunctions

$$
E_{n, \nu}\left(\mathbf{k}_{1}\right)=\frac{1}{\pi \sqrt{2}}\left(\mathbf{k}_{1}^{2}\right)^{i \nu-\frac{1}{2}} e^{i n \phi_{1}}
$$

we can reduce the number of final integrations. To this purpose we define the intermediate coefficients

$$
\begin{aligned}
\hat{C}_{n_{1}, \nu_{1}}^{(1)}\left(\mathbf{k}_{J, 1}, x_{J, 1}\right) & =\int \mathrm{d}^{2} \mathbf{k}_{1} \Phi\left(\mathbf{k}_{J, 1}, x_{J, 1},-\mathbf{k}_{1}\right) E_{n_{1}, \nu_{1}}\left(\mathbf{k}_{1}\right), \\
& =(-1)^{n_{1}} \int \mathrm{d}^{2} \mathbf{k}^{\prime} \Phi\left(\mathbf{k}_{J, 1}, x_{J, 1} \mathbf{k}^{\prime}\right) E_{n_{1}, \nu_{1}}\left(\mathbf{k}^{\prime}\right) \\
\hat{C}_{n_{2}, \nu_{2}}^{(2)}\left(\mathbf{k}_{J, 2}, x_{J, 2}\right) & =\int \mathrm{d}^{2} \mathbf{k}_{2} \Phi\left(\mathbf{k}_{J, 2}, x_{J, 2}, \mathbf{k}_{2}\right) E_{n_{2}, \nu_{2}}^{*}\left(\mathbf{k}_{2}\right),
\end{aligned}
$$

and make use of the following relations between different representations of the BFKL Green's function introducing the - at LL arbitrary - scale $s_{0}$ :

$$
\begin{aligned}
G\left(\mathbf{k}_{1}, \mathbf{k}_{2}, \hat{s}\right) & =\int \frac{\mathrm{d} \omega}{2 \pi i} G_{\omega}\left(\mathbf{k}_{1}, \mathbf{k}_{2}\right)\left(\frac{\hat{s}}{s_{0}}\right)^{\omega}, \\
G_{n_{1}, n_{2}, \nu_{1}, \nu_{2} ; \omega} & =\int \mathrm{d}^{2} \mathbf{k}_{1} \int \mathrm{d}^{2} \mathbf{k}_{2} E_{n_{1}, \nu_{1}}^{*}\left(\mathbf{k}_{1}\right) G_{\omega}\left(\mathbf{k}_{1}, \mathbf{k}_{2}\right) E_{n_{2}, \nu_{2}}\left(\mathbf{k}_{2}\right) \\
& =\frac{1}{\omega-\omega\left(n_{1}, \nu_{1}\right)} \delta_{n_{1}, n_{2}} \delta\left(\nu_{1}-\nu_{2}\right),
\end{aligned}
$$

where $\omega\left(n_{1}, \nu_{1}\right)$ is given by the LL eigenvalue of the BFKL equation, namely

$$
\begin{aligned}
\omega(n, \nu) & =\bar{\alpha}_{s} \chi_{0}\left(|n|, \frac{1}{2}+i \nu\right), \\
\chi_{0}(n, \gamma) & =2 \Psi(1)-\Psi\left(\gamma+\frac{n}{2}\right)-\Psi\left(1-\gamma+\frac{n}{2}\right),
\end{aligned}
$$

with $\Psi(x)=\Gamma^{\prime}(x) / \Gamma(x)$, and $\bar{\alpha}_{s}=N_{c} \alpha_{s} / \pi$.

With these new definitions we can write eq. (2.7) as:

$$
\mathcal{C}_{m} \equiv \sum_{n} \int \mathrm{d} \nu \int \mathrm{d} \phi_{J, 1} \mathrm{~d} \phi_{J, 2} \hat{C}_{n, \nu}^{(1)}\left(\mathbf{k}_{J, 1}, x_{J, 1}\right)\left(\frac{\hat{s}}{s_{0}}\right)^{\omega(n, \nu)} \hat{C}_{n, \nu}^{(2)}\left(\mathbf{k}_{J, 2}, x_{J, 2}\right) \cos (m \varphi)
$$




$$
\begin{aligned}
=(-1)^{m} \sum_{n} \int \mathrm{d} \nu\left(\frac{\hat{s}}{s_{0}}\right)^{\omega(n, \nu)} \\
\times\left[\left(\int \mathrm{d} \phi_{J, 1} \cos \left(m \phi_{J, 1}\right) \hat{C}_{n, \nu}^{(1)}\left(\mathbf{k}_{J, 1}, x_{J, 1}\right)\right)\left(\int \mathrm{d} \phi_{J, 2} \cos \left(m \phi_{J, 2}\right) \hat{C}_{n, \nu}^{(2)}\left(\mathbf{k}_{J, 2}, x_{J, 2}\right)\right)\right. \\
\left.\quad+\left(\int \mathrm{d} \phi_{J, 1} \sin \left(m \phi_{J, 1}\right) \hat{C}_{n, \nu}^{(1)}\left(\mathbf{k}_{J, 1}, x_{J, 1}\right)\right)\left(\int \mathrm{d} \phi_{J, 2} \sin \left(m \phi_{J, 2}\right) \hat{C}_{n, \nu}^{(2)}\left(\mathbf{k}_{J, 2}, x_{J, 2}\right)\right)\right] .
\end{aligned}
$$

After a little bit of simple algebra we end up with

$$
\mathcal{C}_{m}=\left(4-3 \delta_{m, 0}\right) \int \mathrm{d} \nu C_{m, \nu}\left(\left|\mathbf{k}_{J, 1}\right|, x_{J, 1}\right) C_{m, \nu}^{*}\left(\left|\mathbf{k}_{J, 2}\right|, x_{J, 2}\right)\left(\frac{\hat{s}}{s_{0}}\right)^{\omega(m, \nu)} .
$$

Here we have defined

$$
C_{m, \nu}\left(\left|\mathbf{k}_{J}\right|, x_{J}\right)=\int \mathrm{d} \phi_{J} \mathrm{~d}^{2} \mathbf{k} \mathrm{d} x f(x) V(\mathbf{k}, x) E_{m, \nu}(\mathbf{k}) \cos \left(m \phi_{J}\right)
$$

The origin of the factor $\left(4-3 \delta_{m, 0}\right)$ in eq. (2.18) is twofold. Firstly the integration over $\phi_{J}$ leads to a $\delta_{m,|n|}$. Secondly when using the addition formula for $\cos (m \varphi)$ to disentangle $\phi_{J, 1}$ and $\phi_{J, 2}$ also coefficients with sine instead of cosine are generated. While for $m=0$ they vanish, for $m \neq 0$ they give the same contribution as those with the cosine.

Inserting eq. (2.3) into eq. (2.19), we obtain for the LL Mueller Navelet jet vertices in conformal space

$$
C_{m, \nu}^{(\mathrm{LL})}\left(\left|\mathbf{k}_{J}\right|, x_{J}\right)=\frac{\alpha_{s} C_{A / F}}{2}\left(\mathbf{k}_{J}^{2}\right)^{i \nu-1} x_{J} f_{\mathrm{a}}\left(x_{J}\right)\left(1+\delta_{m, 0}\right)
$$

It is worth to note, that $C_{m, \nu}^{(\mathrm{LL})}$ depends on $m$ only in a trivial way $\left(1+\delta_{m, 0}\right)$ such that the azimuthal correlations (2.9) do not depend on the PDFs at all. In the following section we will see, that this changes when one takes into account the NLL corrections to the jet vertices.

\section{NLL calculation}

The master formulae of the LL calculation (2.18), (2.19) will also be used for the NLL calculation. Even though the vertices do not simplify as drastically as in the LL case, we gain the possibility to calculate for a limited number of $m$ the coefficients $C_{m, \nu}$ as universal grids in $\nu$. In transverse momentum space one would need a two dimensional grid. Moreover, at NLL there are some contributions with an additional transverse momentum integration, such that some contributions would be analytic functions in e.g. $\mathbf{k}_{1}$ while other would be proportional to distributions like $\delta^{(2)}\left(\mathbf{k}_{1}-\mathbf{k}_{J, 1}\right)$. 


\subsection{Strong coupling, renormalization scheme and PDFs at NLL}

Based on the $\overline{\mathrm{MS}}$ renormalization scheme, we use the MSTW 2008 PDFs [16] and the two-loop strong coupling in the following form:

$$
\alpha_{s}\left(\mu_{R}^{2}\right)=\frac{1}{b_{0} L}\left(1+\frac{b_{1}}{b_{0}^{2}} \frac{\ln L}{L}\right)
$$

with $L=\ln \mu_{R}^{2} / \Lambda_{\mathrm{QCD}}^{2}$, and

$$
b_{0}=\frac{33-2 N_{f}}{12 \pi}, \quad b_{1}=\frac{153-19 N_{f}}{24 \pi^{2}} .
$$

In the following $\alpha_{s}$ or $\bar{\alpha}_{s}$ without argument is to be understood as $\alpha_{s}\left(\mu_{R}^{2}\right)$ or $\bar{\alpha}_{s}\left(\mu_{R}^{2}\right)$ respectively. Since in the MSTW 2008 PDFs $\mu_{R}$ and $\mu_{F}$ are set to be equal, for a consistent calculation we are forced to perform this identification throughout the whole calculation as well.

\subsection{Jet vertices at NLL}

To calculate the coefficients $C_{m, \nu}(2.19)$ at next to leading order level, we take for $V_{\mathrm{a}}(\mathbf{k}, x)$ instead of just the leading order result $V^{(0)}(\mathbf{k}, x)(2.3)$ the full NLL vertex

$$
V_{\mathrm{a}}(\mathbf{k}, x)=V_{\mathrm{a}}^{(0)}(\mathbf{k}, x)+\alpha_{s} V_{\mathrm{a}}^{(1)}(\mathbf{k}, x) .
$$

The matrix elements needed to calculate the Mueller Navelet jet vertex at next to leading order - namely the partonic $2 \rightarrow 3$ process at tree level and the partonic $2 \rightarrow 2$ process at one loop level - are known for a long time. The separation of collinear singularities (to be absorbed by renormalized PDFs) from the BFKL large logarithms in $s$ was performed by Bartels, Vacca and one of us $[13,14]$ in terms of a generic and infrared-safe jet algorithm. In this paper, we shall apply such procedure to a concrete jet algorithm, namely the cone algorithm, as will be explained in section 3.2.1.

We will build on the results obtained in ref. $[13,14]$ using their notation as well, but we correct an inconsistency in the treatment of the collinear cutoff parameter $\Lambda$ which later is identified with the factorization scale $\mu_{F}$. In the 'real' $C_{F}$ term the authors rescale the transverse momentum which is integrated over but do not adapt the cutoff parameter $\Lambda$. The correction of this point does not change the singular terms, and all the discussion of the arrangement of divergences and subtractions remains unchanged. However the finite part of the subtraction changes such that beside the cutoff functions also the 'virtual' part of the vertex changes, e.g. the term proportional to $\left(\frac{\ln (1-z)}{1-z}\right)_{+}$vanishes completely. ${ }^{1}$

The final expressions for the NLL correction to the vertices read:

$$
\begin{aligned}
& V_{\mathrm{g}}^{(1)}(\mathbf{k}, x) \\
& =\left[\left(\frac{11}{6} \frac{C_{A}}{\pi}-\frac{1}{3} \frac{N_{f}}{\pi}\right) \ln \frac{\mathbf{k}^{2}}{\Lambda^{2}}+\left(\frac{\pi^{2}}{4}-\frac{67}{36}\right) \frac{C_{A}}{\pi}+\frac{13}{36} \frac{N_{f}}{\pi}-b_{0} \ln \frac{\mathbf{k}^{2}}{\mu^{2}}\right] V_{\mathrm{g}}^{(0)}(\mathbf{k}, x)
\end{aligned}
$$

\footnotetext{
${ }^{1}$ We note a misprint in equation (105) of ref. [13]: in the 'real' $C_{A}$ term the expression $\mathbf{q}-\mathbf{k}$ must be replaced by $\mathbf{q}-z \mathbf{k}$ both in numerator and in the denominator. Just after it, +- is to be interpreted as - .
} 


$$
\begin{aligned}
& +\int \mathrm{d} z \frac{N_{f}}{\pi} \frac{C_{F}}{C_{A}} z(1-z) V_{\mathrm{g}}^{(0)}(\mathbf{k}, x z) \\
& +\frac{N_{f}}{\pi} \int \frac{\mathrm{d}^{2} \mathbf{k}^{\prime}}{\pi} \int_{0}^{1} \mathrm{~d} z P_{\mathrm{qg}}(z)\left[\frac{h_{\mathrm{q}}^{(0)}\left(\mathbf{k}^{\prime}\right)}{\left(\mathbf{k}-\mathbf{k}^{\prime}\right)^{2}+\mathbf{k}^{\prime 2}} \mathcal{S}_{J}^{(3)}\left(\mathbf{k}^{\prime}, \mathbf{k}-\mathbf{k}^{\prime}, x z ; x\right)\right. \\
& \left.-\frac{1}{\mathbf{k}^{\prime 2}} \Theta\left(\Lambda^{2}-\mathbf{k}^{\prime 2}\right) V_{\mathrm{q}}^{(0)}(\mathbf{k}, x z)\right] \\
& +\frac{N_{f}}{2 \pi} \int \frac{\mathrm{d}^{2} \mathbf{k}^{\prime}}{\pi} \int_{0}^{1} \mathrm{~d} z P_{\mathrm{qg}}(z) \frac{\mathcal{N} C_{A}}{\left((1-z) \mathbf{k}-\mathbf{k}^{\prime}\right)^{2}}\left[z(1-z) \frac{\left(\mathbf{k}-\mathbf{k}^{\prime}\right) \cdot \mathbf{k}^{\prime}}{\left(\mathbf{k}-\mathbf{k}^{\prime}\right)^{2} \mathbf{k}^{\prime 2}} \mathcal{S}_{J}^{(3)}\left(\mathbf{k}^{\prime}, \mathbf{k}-\mathbf{k}^{\prime}, x z ; x\right)\right. \\
& \left.-\frac{1}{\mathbf{k}^{2}} \Theta\left(\Lambda^{2}-\left((1-z) \mathbf{k}-\mathbf{k}^{\prime}\right)^{2}\right) \mathcal{S}_{J}^{(2)}(\mathbf{k}, x)\right] \\
& +\frac{C_{A}}{\pi} \int_{0}^{1} \frac{\mathrm{d} z}{1-z}[(1-z) P(1-z)] \int \frac{\mathrm{d}^{2} \mathbf{l}}{\pi \mathbf{l}^{2}} \\
& \times\left\{\frac { \mathcal { N } C _ { A } } { \mathbf { l } ^ { 2 } + ( \mathbf { l } - \mathbf { k } ) ^ { 2 } } \left[\mathcal{S}_{J}^{(3)}(z \mathbf{k}+(1-z) \mathbf{l},(1-z)(\mathbf{k}-\mathbf{l}), x(1-z) ; x)\right.\right. \\
& \left.+\mathcal{S}_{J}^{(3)}(\mathbf{k}-(1-z) \mathbf{l},(1-z) \mathbf{l}, x(1-z) ; x)\right] \\
& \left.-\Theta\left(\frac{\Lambda^{2}}{(1-z)^{2}}-\mathbf{l}^{2}\right)\left[V_{\mathrm{g}}^{(0)}(\mathbf{k}, x)+V_{\mathrm{g}}^{(0)}(\mathbf{k}, x z)\right]\right\} \\
& -\frac{2 C_{A}}{\pi} \int_{0}^{1} \frac{\mathrm{d} z}{1-z} \int \frac{\mathrm{d}^{2} \mathbf{l}}{\pi \mathbf{l}^{2}}\left[\frac{\mathcal{N} C_{A}}{\mathbf{l}^{2}+(\mathbf{l}-\mathbf{k})^{2}} S_{J}^{(2)}(\mathbf{k}, x)-\Theta\left(\frac{\Lambda^{2}}{(1-z)^{2}}-\mathbf{l}^{2}\right) V_{\mathrm{g}}^{(0)}(\mathbf{k}, x)\right] \\
& +\frac{C_{A}}{\pi} \int \frac{\mathrm{d}^{2} \mathbf{k}^{\prime}}{\pi} \int_{0}^{1} \mathrm{~d} z\left[P ( z ) \left((1-z) \frac{\left(\mathbf{k}-\mathbf{k}^{\prime}\right) \cdot\left((1-z) \mathbf{k}-\mathbf{k}^{\prime}\right)}{\left(\mathbf{k}-\mathbf{k}^{\prime}\right)^{2}\left((1-z) \mathbf{k}-\mathbf{k}^{\prime}\right)^{2}} h_{\mathrm{g}}^{(0)}\left(\mathbf{k}^{\prime}\right)\right.\right. \\
& \left.\times \mathcal{S}_{J}^{(3)}\left(\mathbf{k}^{\prime}, \mathbf{k}-\mathbf{k}^{\prime}, x z ; x\right)-\frac{1}{\mathbf{k}^{\prime 2}} \Theta\left(\Lambda^{2}-\mathbf{k}^{\prime 2}\right) V_{\mathrm{g}}^{(0)}(\mathbf{k}, x z)\right) \\
& \left.-\frac{1}{z\left(\mathbf{k}-\mathbf{k}^{\prime}\right)^{2}} \Theta\left(\left|\mathbf{k}-\mathbf{k}^{\prime}\right|-z\left(\left|\mathbf{k}-\mathbf{k}^{\prime}\right|+\left|\mathbf{k}^{\prime}\right|\right)\right) V_{\mathrm{g}}^{(0)}\left(\mathbf{k}^{\prime}, x\right)\right] \text {. }
\end{aligned}
$$

$$
\begin{aligned}
& V_{\mathrm{q}}^{(1)}(\mathbf{k}, x) \\
& =\left[\left(\frac{3}{2} \ln \frac{\mathbf{k}^{2}}{\Lambda^{2}}-\frac{15}{4}\right) \frac{C_{F}}{\pi}+\left(\frac{85}{36}+\frac{\pi^{2}}{4}\right) \frac{C_{A}}{\pi}-\frac{5}{18} \frac{N_{f}}{\pi}-b_{0} \ln \frac{\mathbf{k}^{2}}{\mu^{2}}\right] V_{\mathrm{q}}^{(0)}(\mathbf{k}, x) \\
& +\int \mathrm{d} z\left(\frac{C_{F}}{\pi} \frac{1-z}{2}+\frac{C_{A}}{\pi} \frac{z}{2}\right) V_{\mathrm{q}}^{(0)}(\mathbf{k}, x z) \\
& +\frac{C_{A}}{\pi} \int \frac{\mathrm{d}^{2} \mathbf{k}^{\prime}}{\pi} \int \mathrm{d} z\left[\frac{1+(1-z)^{2}}{2 z}\right. \\
& \quad \times\left((1-z) \frac{\left(\mathbf{k}-\mathbf{k}^{\prime}\right) \cdot\left((1-z) \mathbf{k}-\mathbf{k}^{\prime}\right)}{\left(\mathbf{k}-\mathbf{k}^{\prime}\right)^{2}\left((1-z) \mathbf{k}-\mathbf{k}^{\prime}\right)^{2}} h_{\mathrm{q}}^{(0)}\left(\mathbf{k}^{\prime}\right) \mathcal{S}_{J}^{(3)}\left(\mathbf{k}^{\prime}, \mathbf{k}-\mathbf{k}^{\prime}, x z ; x\right)\right. \\
& \left.\quad-\frac{1}{\mathbf{k}^{\prime 2}} \Theta\left(\Lambda^{2}-\mathbf{k}^{\prime 2}\right) V_{\mathrm{q}}^{(0)}(\mathbf{k}, x z)\right)
\end{aligned}
$$




$$
\begin{gathered}
\left.-\frac{1}{z\left(\mathbf{k}-\mathbf{k}^{\prime}\right)^{2}} \Theta\left(\left|\mathbf{k}-\mathbf{k}^{\prime}\right|-z\left(\left|\mathbf{k}-\mathbf{k}^{\prime}\right|+\left|\mathbf{k}^{\prime}\right|\right)\right) V_{\mathrm{q}}^{(0)}\left(\mathbf{k}^{\prime}, x\right)\right] \\
+\frac{C_{F}}{2 \pi} \int \mathrm{d} z \frac{1+z^{2}}{1-z} \int \frac{\mathrm{d}^{2} \mathbf{l}}{\pi \mathbf{l}^{2}} \\
\times\left[\frac { \mathcal { N } C _ { F } } { \mathbf { l } ^ { 2 } + ( \mathbf { l } - \mathbf { k } ) ^ { 2 } } \left(\mathcal{S}_{J}^{(3)}(z \mathbf{k}+(1-z) \mathbf{l},(1-z)(\mathbf{k}-\mathbf{l}), x(1-z) ; x)\right.\right. \\
\left.+\mathcal{S}_{J}^{(3)}(\mathbf{k}-(1-z) \mathbf{l},(1-z) \mathbf{l}, x(1-z) ; x)\right) \\
\left.-\Theta\left(\frac{\Lambda^{2}}{(1-z)^{2}}-\mathbf{l}^{2}\right)\left(V_{\mathrm{q}}^{(0)}(\mathbf{k}, x)+V_{\mathrm{q}}^{(0)}(\mathbf{k}, x z)\right)\right] \\
-\frac{2 C_{F}}{\pi} \int \mathrm{d} z\left(\frac{1}{1-z}\right) \int \frac{\mathrm{d}^{2} \mathbf{l}}{\pi \mathbf{l}^{2}}\left[\frac{\mathcal{N} C_{F}}{\mathbf{l}^{2}+(\mathbf{l}-\mathbf{k})^{2}} S_{J}^{(2)}(\mathbf{k}, x)\right. \\
\left.-\Theta\left(\frac{\Lambda^{2}}{(1-z)^{2}}-\mathbf{l}^{2}\right) V_{\mathrm{q}}^{(0)}(\mathbf{k}, x)\right]
\end{gathered}
$$

Here $N_{f}$ denotes the number of active quark flavors, $b_{0}=\left(11 N_{c}-2 N_{f}\right) /(12 \pi)$, and $\mathcal{N}=$ $\alpha_{s} / \sqrt{2}$. A priori, the factorization scale $\mu_{F}=\Lambda$ and the renormalization scale $\mu_{R}=\mu$ are independent of each other even though in the end we will set them equal.

\subsubsection{Jet definition}

For a concrete calculation of Mueller Navelet jet production one also has to choose a concrete jet algorithm obeying the property of infra-red safety, as required by the general procedure of refs. $[13,14]$. Two of the most common ones are the cone algorithm

$$
\begin{aligned}
\mathcal{S}_{J}^{(3, \text { cone })}\left(\mathbf{k}^{\prime}, \mathbf{k}-\right. & \left.\mathbf{k}^{\prime}, x z ; x\right)=\mathcal{S}_{J}^{(2)}(\mathbf{k}, x) \Theta\left(\left[\frac{\left|\mathbf{k}-\mathbf{k}^{\prime}\right|+\left|\mathbf{k}^{\prime}\right|}{\max \left(\left|\mathbf{k}-\mathbf{k}^{\prime}\right|,\left|\mathbf{k}^{\prime}\right|\right)} R_{\text {cone }}\right]^{2}-\left[\Delta y^{2}+\Delta \phi^{2}\right]\right) \\
& +\mathcal{S}_{J}^{(2)}\left(\mathbf{k}-\mathbf{k}^{\prime}, x z\right) \Theta\left(\left[\Delta y^{2}+\Delta \phi^{2}\right]-\left[\frac{\left|\mathbf{k}-\mathbf{k}^{\prime}\right|+\left|\mathbf{k}^{\prime}\right|}{\max \left(\left|\mathbf{k}-\mathbf{k}^{\prime}\right|,\left|\mathbf{k}^{\prime}\right|\right)} R_{\text {cone }}\right]^{2}\right) \\
& +\mathcal{S}_{J}^{(2)}\left(\mathbf{k}^{\prime}, x(1-z)\right) \Theta\left(\left[\Delta y^{2}+\Delta \phi^{2}\right]-\left[\frac{\left|\mathbf{k}-\mathbf{k}^{\prime}\right|+\left|\mathbf{k}^{\prime}\right|}{\max \left(\left|\mathbf{k}-\mathbf{k}^{\prime}\right|,\left|\mathbf{k}^{\prime}\right|\right)} R_{\text {cone }}\right]^{2}\right),
\end{aligned}
$$

as it has been adapted for NLL calculation in ref. [17], and the $k_{T}$ algorithm

$$
\begin{aligned}
\mathcal{S}_{J}^{\left(3, k_{T}\right)}\left(\mathbf{k}^{\prime}, \mathbf{k}-\mathbf{k}^{\prime}, x z ; x\right)= & \mathcal{S}_{J}^{(2)}(\mathbf{k}, x) \Theta\left(R_{k_{T}}^{2}-\left[\Delta y^{2}+\Delta \phi^{2}\right]\right) \\
& +\mathcal{S}_{J}^{(2)}\left(\mathbf{k}-\mathbf{k}^{\prime}, x z\right) \Theta\left(\left[\Delta y^{2}+\Delta \phi^{2}\right]-R_{k_{T}}^{2}\right) \\
& +\mathcal{S}_{J}^{(2)}\left(\mathbf{k}^{\prime}, x(1-z)\right) \Theta\left(\left[\Delta y^{2}+\Delta \phi^{2}\right]-R_{k_{T}}^{2}\right),
\end{aligned}
$$

where

$$
\Delta y=\log \left(\frac{1-z}{z} \frac{\left|\mathbf{k}-\mathbf{k}^{\prime}\right|}{\left|\mathbf{k}^{\prime}\right|}\right), \quad \Delta \phi=\arccos \frac{\mathbf{k}^{\prime}\left(\mathbf{k}-\mathbf{k}^{\prime}\right)}{\sqrt{\mathbf{k}^{\prime 2}\left(\mathbf{k}-\mathbf{k}^{\prime}\right)^{2}}}
$$


In our study we will use the cone algorithm with a cone size of $R_{\text {cone }}=0.5$ as it probably will be used in a CMS analysis at the LHC [15].

\subsubsection{LL subtraction and $s_{0}$}

The requirement of a BFKL calculation that the two scattering objects have a similar hard scale is reflected by the fact that in this standard situation of BFKL physics the energy scale $s_{0}$ can be written as a product of two energy scales each assigned to one of these scattering objects.

$$
s_{0}=\sqrt{s_{0,1} s_{0,2}} .
$$

In ref. $[13,14]$ the energy scale $s_{0, i}$ (assigned to the Mueller Navelet jet) was chosen as $\left(\left|\mathbf{k}_{J}\right|+\left|\mathbf{k}_{J}-\mathbf{k}\right|\right)^{2}$. While $\mathbf{k}$ is integrated over, it is preferable to let $s_{0}$ depend only on external scales. Also $\hat{s}=x_{1} x_{2} s$ is in fact not an external scale since the longitudinal momentum fractions $x_{1}$ and $x_{2}$ are integrated over as well. Therefore, we want to change to a new $s_{0}^{\prime}$ :

$$
\begin{aligned}
s_{0,1}=\left(\left|\mathbf{k}_{J, 1}\right|+\left|\mathbf{k}_{J, 1}-\mathbf{k}_{1}\right|\right)^{2} & \rightarrow s_{0,1}^{\prime}=\frac{x_{1}^{2}}{x_{J, 1}^{2}} \mathbf{k}_{J, 1}^{2}, \\
s_{0,2}=\left(\left|\mathbf{k}_{J, 2}\right|+\left|\mathbf{k}_{J, 2}-\mathbf{k}_{2}\right|\right)^{2} & \rightarrow s_{0,2}^{\prime}=\frac{x_{2}^{2}}{x_{J, 2}^{2} \mathbf{k}_{J, 2}^{2},} \\
\frac{\hat{s}}{s_{0}} & \rightarrow \frac{\hat{s}}{s_{0}^{\prime}}=\frac{x_{J, 1} x_{J_{2}} s}{\left|\mathbf{k}_{J, 1}\right| \cdot\left|\mathbf{k}_{J, 2}\right|}=e^{y_{J, 1}-y_{J, 2}} \equiv e^{Y},
\end{aligned}
$$

where we introduced the relative rapidity $Y=y_{J, 1}-y_{J, 2}$.

The energy scale $s_{0}$ is a free parameter in the calculation. However, like for the renormalization scale at NLL level a change of it does not go without consequences. In fact, a change of $s_{0} \rightarrow s_{0}^{\prime}$ in the Green's function has to be accompanied by an according correction term to the impact factors $[18,19]$ :

$$
\Phi_{\mathrm{NLL}}\left(\mathbf{k}_{i} ; s_{0, i}^{\prime}\right)=\Phi_{\mathrm{NLL}}\left(\mathbf{k}_{i} ; s_{0, i}\right)+\int \mathrm{d}^{2} \mathbf{k}^{\prime} \Phi_{\mathrm{LL}}\left(\mathbf{k}_{i}^{\prime}\right) \mathcal{K}_{\mathrm{LL}}\left(\mathbf{k}_{i}^{\prime}, \mathbf{k}_{i}\right) \frac{1}{2} \ln \frac{s_{0, i}^{\prime}}{s_{0, i}}
$$

with $\mathcal{K}_{\mathrm{LL}}$ being the LL BFKL kernel. Due to the Dirac delta distribution $\delta\left(1-x_{J, i} / x_{i}\right)$ in the jet algorithm inside $\Phi$ the ratio of longitudinal momentum fractions in $s_{0, i}^{\prime}$ reduces to 1 and hence the logarithm in eq. (3.13) vanishes for $\mathbf{k}_{i}^{\prime}=\mathbf{k}_{i}$ such that only the real part of the kernel contributes.

To study the role of $s_{0}$, we will investigate the effect when changing it. A subsequent change of $s_{0, i}$ by just a factor $\lambda$ can be easily performed at the very end because of the use of BFKL eigenfunctions:

$$
\begin{aligned}
& C_{m, \nu}\left(\left|\mathbf{k}_{J}\right|, x_{J} ; s_{0}^{\prime \prime}=\lambda s_{0}^{\prime}\right)-C_{m, \nu}\left(\left|\mathbf{k}_{J}\right|, x_{J} ; s_{0}^{\prime}\right) \\
& =\int \mathrm{d} \phi_{J} \mathrm{~d}^{2} \mathbf{k} \int \mathrm{d}^{2} \mathbf{k}^{\prime} \mathrm{d} x f(x) V^{(0)}\left(\mathbf{k}^{\prime}, x\right) \mathcal{K}\left(\mathbf{k}^{\prime}, \mathbf{k}\right) E_{m, \nu}(\mathbf{k}) \cos \left(m \phi_{J}\right) \frac{1}{2} \ln \frac{s_{0}^{\prime \prime}}{s_{0}^{\prime}} \\
& =\int \mathrm{d} \phi_{J} \int \mathrm{d}^{2} \mathbf{k}^{\prime} \mathrm{d} x f(x) V^{(0)}\left(\mathbf{k}^{\prime}, x\right) \bar{\alpha}_{s} \chi_{0}\left(m, \frac{1}{2}+i \nu\right) E_{m, \nu}\left(\mathbf{k}^{\prime}\right) \cos \left(m \phi_{J}\right) \frac{1}{2} \ln \lambda \\
& =\bar{\alpha}_{s} \chi_{0}\left(m, \frac{1}{2}+i \nu\right) C_{m, \nu}^{(\mathrm{LL})}\left(\left|\mathbf{k}_{J}\right|, x_{J}\right) \frac{1}{2} \ln \lambda .
\end{aligned}
$$


The LL subtraction, i.e. the terms multiplied by $\Theta\left(\left|\mathbf{k}-\mathbf{k}^{\prime}\right|-z\left(\left|\mathbf{k}-\mathbf{k}^{\prime}\right|+\left|\mathbf{k}^{\prime}\right|\right)\right)$ in eqs. (3.5), (3.4), cancels some part in the limit of the additional emission having a big rapidity distance to the jet. In fact, numerically this cancellation works very poorly due to an azimuthal averaging which has been performed for the LL subtraction. A significant improvement can be obtained by omitting this averaging and introducing new LL subtraction terms

$$
\begin{aligned}
& V_{\mathrm{q} ; \text { LL subtraction }}^{(1)}=-\frac{C_{A}}{\pi^{2}} \frac{1}{z\left(\mathbf{k}-\mathbf{k}^{\prime}\right)^{2}} \frac{\left(\mathbf{k}-\mathbf{k}^{\prime}\right)\left(\mathbf{k}-\mathbf{k}^{\prime}-z \mathbf{k}^{\prime}\right)}{\left(\mathbf{k}-\mathbf{k}^{\prime}\right)^{2}\left(\mathbf{k}-\mathbf{k}^{\prime}-z \mathbf{k}^{\prime}\right)^{2}} V_{\mathrm{q}}^{(0)}\left(\mathbf{k}^{\prime}, x\right), \\
& V_{\mathrm{g} ; \text { LL subtraction }}^{(1)}=-\frac{C_{A}}{\pi^{2}} \frac{1}{z\left(\mathbf{k}-\mathbf{k}^{\prime}\right)^{2}} \frac{\left(\mathbf{k}-\mathbf{k}^{\prime}\right)\left(\mathbf{k}-\mathbf{k}^{\prime}-z \mathbf{k}^{\prime}\right)}{\left(\mathbf{k}-\mathbf{k}^{\prime}\right)^{2}\left(\mathbf{k}-\mathbf{k}^{\prime}-z \mathbf{k}^{\prime}\right)^{2}} V_{\mathrm{g}}^{(0)}\left(\mathbf{k}^{\prime}, x\right) .
\end{aligned}
$$

As a consequence $s_{0, i}$ changes from $s_{0, i}=\left(\left|\mathbf{k}_{J, i}\right|+\left|\mathbf{k}_{J, i}-\mathbf{k}_{i}\right|\right)^{2}$ to $s_{0, i}=\left(\mathbf{k}_{i}-2 \mathbf{k}_{J, i}\right)^{2}$. It is also possible to use

$$
\begin{aligned}
& \widetilde{V}_{\mathrm{q} ; \text { LL subtraction }}^{(1)}=-\frac{C_{A}}{\pi^{2}} \frac{1}{z\left(\mathbf{k}-\mathbf{k}^{\prime}\right)^{2}} \frac{\left(\mathbf{k}-\mathbf{k}^{\prime}\right)\left(\mathbf{k}-\mathbf{k}^{\prime}-z \mathbf{k}\right)}{\left(\mathbf{k}-\mathbf{k}^{\prime}\right)^{2}\left(\mathbf{k}-\mathbf{k}^{\prime}-z \mathbf{k}\right)^{2}} V_{\mathrm{q}}^{(0)}\left(\mathbf{k}^{\prime}, x\right), \\
& \widetilde{V}_{\mathrm{g} ; \text { LL subtraction }}^{(1)}=-\frac{C_{A}}{\pi^{2}} \frac{1}{z\left(\mathbf{k}-\mathbf{k}^{\prime}\right)^{2}} \frac{\left(\mathbf{k}-\mathbf{k}^{\prime}\right)\left(\mathbf{k}-\mathbf{k}^{\prime}-z \mathbf{k}\right)}{\left(\mathbf{k}-\mathbf{k}^{\prime}\right)^{2}\left(\mathbf{k}-\mathbf{k}^{\prime}-z \mathbf{k}\right)^{2}} V_{\mathrm{g}}^{(0)}\left(\mathbf{k}^{\prime}, x\right),
\end{aligned}
$$

which are slightly inferior concerning the numerical performance but give a $s_{0}$ change from $s_{0, i}=\left(\left|\mathbf{k}_{J, i}\right|+\left|\mathbf{k}_{J, i}-\mathbf{k}_{i}\right|\right)^{2}$ to $s_{0, i}=\mathbf{k}_{J, i}^{2}$ which already is close to the final $s_{0}^{\prime}$ making a correction term (eq. (3.13)) needless since the ratio of longitudinal momentum fractions in $s_{0, i}^{\prime}$ effectively reduces to 1 as described above.

We have checked that all three possible subtraction terms after combining them with the according correction term (3.13) lead to the same result. For reasons of numerical performance we have chosen eqs. (3.15) for the final calculation. As we nevertheless aim for the final $s_{0}^{\prime}$ defined in eq. (3.12) we still have to use the correction term introduced in eq. (3.13) additionally.

\subsection{BFKL Green's function at NLL}

Last but not least, we also have to take the BFKL Green's function at NLL level. The key to the Green's function is the BFKL kernel at NLL $[20,21]$. While at LL the BFKL equation is conformally invariant, at NLL it is not such that in fact the LL eigenfunctions $E_{n, \nu}(2.10)$ are strictly speaking not eigenfunctions of the NLL kernel. Nevertheless, the action of the NLL BFKL kernel on the eigenfunctions has been calculated in ref. [22]. The status of the $E_{n, \nu}$ being eigenfunctions formally can be saved if one accepts the eigenvalue to become an operator containing a derivative with respect to $\nu[11,23,24]$. In combination with the impact factors the derivate acts on the impact factors and effectively leads to a contribution to the eigenvalue which depends on the impact factors [11, 23-25]:

$$
\begin{aligned}
\omega(n, \nu)=\bar{\alpha}_{s} \chi_{0}( & \left.|n|, \frac{1}{2}+i \nu\right)+\bar{\alpha}_{s}^{2}\left[\chi_{1}\left(|n|, \frac{1}{2}+i \nu\right)\right. \\
& \left.-\frac{\pi b_{0}}{2 N_{c}} \chi_{0}\left(|n|, \frac{1}{2}+i \nu\right)\left\{-2 \ln \mu_{R}^{2}-i \frac{\partial}{\partial \nu} \ln \frac{C_{n, \nu}\left(\left|\mathbf{k}_{J, 1}\right|, x_{J, 1}\right)}{C_{n, \nu}\left(\left|\mathbf{k}_{J, 2}\right|, x_{J, 2}\right)}\right\}\right],
\end{aligned}
$$


where

$$
\begin{aligned}
& \chi_{1}(n, \gamma)= \mathcal{S} \chi_{0}(n, \gamma)+\frac{3}{2} \zeta(3)-\frac{\beta_{0}}{8 N_{c}} \chi_{0}^{2}(n, \gamma) \\
&+\frac{1}{4}\left[\psi^{\prime \prime}\left(\gamma+\frac{n}{2}\right)+\psi^{\prime \prime}\left(1-\gamma+\frac{n}{2}\right)-2 \phi(n, \gamma)-2 \phi(n, 1-\gamma)\right] \\
&-\frac{\pi^{2} \cos (\pi \gamma)}{4 \sin ^{2}(\pi \gamma)(1-2 \gamma)}\left\{\left[3+\left(1+\frac{N_{f}}{N_{c}^{3}}\right) \frac{2+3 \gamma(1-\gamma)}{(3-2 \gamma)(1+2 \gamma)}\right] \delta_{n, 0}\right. \\
&\left.-\left(1+\frac{N_{f}}{N_{c}^{3}}\right) \frac{\gamma(1-\gamma)}{2(3-2 \gamma)(1+2 \gamma)} \delta_{n, 2}\right\},
\end{aligned}
$$

with the constant $\mathcal{S}=\left(4-\pi^{2}+5 \beta_{0} / N_{c}\right) / 12 . \quad \zeta(n)=\sum_{k=1}^{\infty} k^{-n}$ is the Riemann zeta function while the function $\phi$ reads

$$
\begin{aligned}
\phi(n, \gamma)=\sum_{k=0}^{\infty} & \frac{(-1)^{k+1}}{k+\gamma+\frac{n}{2}}\left(\psi^{\prime}(k+n+1)-\psi^{\prime}(k+1)\right. \\
& \left.\quad+(-1)^{k+1}\left[\beta^{\prime}(k+n+1)+\beta^{\prime}(k+1)\right]+\frac{\psi(k+1)-\psi(k+n+1)}{k+\gamma+\frac{n}{2}}\right),
\end{aligned}
$$

with

$$
\beta^{\prime}(\gamma)=\frac{1}{4}\left[\psi^{\prime}\left(\frac{1+\gamma}{2}\right)-\psi^{\prime}\left(\frac{\gamma}{2}\right)\right] .
$$

At NLL accuracy, only the leading order vertex coefficients (2.20) enter in the derivative term of (3.17):

$$
-2 \ln \mu_{R}^{2}-i \frac{\partial}{\partial \nu} \ln \frac{C_{n, \nu}^{(\mathrm{LL})}\left(\left|\mathbf{k}_{J, 1}\right|, x_{J, 1}\right)}{\left(C_{n, \nu}^{(\mathrm{LL})}\left(\left|\mathbf{k}_{J, 2}\right|, x_{J, 2}\right)\right)^{*}}=2 \ln \frac{\left|\mathbf{k}_{J, 1}\right| \cdot\left|\mathbf{k}_{J, 2}\right|}{\mu_{R}^{2}} .
$$

\subsubsection{Collinear improved Green's function}

There are methods to improve the NLL BFKL kernel for $n=0$ by imposing compatibility with the DGLAP equation [26-29] in the collinear limit [30-33]. They are known under the name $\omega$-shift because essentially poles in $\gamma=1 / 2+i \nu$ and $1-\gamma$ are shifted by $\omega / 2$ with some compensation terms ensuring that the result is not changed at fixed order (having in mind that $\omega \sim \bar{\alpha}_{s} \chi_{0}$ ). The different attempts are very similar, and here we use the most transparent method presented in [30]. In fact, based on previous experience $[11,25,34]$ we use scheme 3 of [30]. The new kernel $\bar{\alpha}_{s} \chi^{(1)}(\gamma, \omega)$ with shifted poles replaces $\bar{\alpha}_{s} \chi_{0}(\gamma, 0)+\bar{\alpha}_{s}^{2} \chi_{1}(\gamma, 0)$ and $\omega(0, \nu)$ is obtained by solving the implicit equation

$$
\omega(0, \nu)=\bar{\alpha}_{s} \chi^{(1)}(\gamma, \omega(0, \nu))
$$

for $\omega(n, \nu)$ numerically.

In general the additional $\nu$-derivative term makes it necessary to recalculate the coefficients $d_{1, k}$ (defined in ref. [30]) but in our case the LL vertex does not contain any poles in 
$\gamma$ nor in $1-\gamma$ leaving the coefficients $d_{1, k}$ unchanged. ${ }^{2}$ By introducing an $\omega$ dependence in the eigenvalue the pole in (2.14) is no longer a simple one such that the residue in fact reads

$$
G_{0,0, \nu_{1}, \nu_{2}}(\hat{s})=\left(1-\left.\frac{\partial \chi^{(1)}\left(\frac{1}{2}+i \nu_{1}, \omega\right)}{\partial \omega}\right|_{\omega=\omega\left(0, \nu_{1}\right)}\right)^{-1}\left(\frac{\hat{s}}{s_{0}}\right)^{\omega\left(0, \nu_{1}\right)} \delta\left(\nu_{1}-\nu_{2}\right) .
$$

\subsubsection{Approximate energy-momentum conservation in BFKL}

We would like to finish this section with the following important observation. Energymomentum conservation is not fulfilled in any truncated BFKL treatment (i.e. LL BFKL, or NLL BFKL, etc.) while it is preserved in any truncated fixed order treatment à la DGLAP (LO, NLO, etc.). Our approach uses a semi-analytical resummed solution of the BFKL equation at NLL and does not allow, in a direct way, for the implementation of a procedure based on the iteration of the BFKL kernel (in the spirit of ref. [9]) in which energy-momentum conservation could be imposed step by step. Exact energy-momentum conservation is beyond the scope of our pure BFKL treatment. Therefore, our results are expected to be valid only in a limited range of relative rapidity between the two Mueller Navelet jets, away from the kinematical bounds. Nevertheless, this violation within BFKL approach is less dramatic when going further in the truncation (note that BFKL and DGLAP are expected to converge to same result when going higher and higher in the order of perturbation). We thus expect that in the region far from the kinematical limit, such energy-momentum conservation effects would not introduce significant corrections to our NLL BFKL results.

\section{Results}

We now present results for the LHC at the design center of mass energy $\sqrt{s}=14 \mathrm{TeV}$. Motivated by a recent CMS study [15] we restrict the rapidities of the Mueller Navelet jets to the region $3<\left|y_{J}\right|<5$. We shall show the differential cross section with respect to the relative rapidity variable $Y=y_{J, 1}-y_{J, 2}$ which therefore takes values between 6 and 10 . Note that, since the maximum possible rapidity of a jet with $50 \mathrm{GeV}$ of transverse energy (see below) is $y_{\max }=5.6$, values of $Y \simeq 10$ are quite close to the kinematical boundary and the corresponding predictions may suffer some uncertainties because of the fact that momentum conservation is not exactly fulfilled in this BFKL approach.

We consider Mueller Navelet jets with $\left|\mathbf{k}_{J}\right|=35 \mathrm{GeV}$, and $\left|\mathbf{k}_{J}\right|=50 \mathrm{GeV}$ respectively. Due to our method of calculation and the factorization between the two Mueller Navelet jets we can can combine the building blocks to two symmetric scenarios $\left(\left|\mathbf{k}_{J, 1}\right|=\left|\mathbf{k}_{J, 2}\right|=\right.$ $35 \mathrm{GeV}$ or $\left|\mathbf{k}_{J, 1}\right|=\left|\mathbf{k}_{J, 2}\right|=50 \mathrm{GeV}$ ) and one asymmetric scenario of $\left|\mathbf{k}_{J, 1}\right|=35 \mathrm{GeV}$, $\left|\mathbf{k}_{J, 2}\right|=50 \mathrm{GeV}$ (plus the 'mirrored' process $\left|\mathbf{k}_{J, 1}\right|=50 \mathrm{GeV},\left|\mathbf{k}_{J, 2}\right|=35 \mathrm{GeV}$ ) even though

\footnotetext{
${ }^{2}$ The same is true for the NLL vertex (except for the $s_{0}$-correction term (3.13)) as can be seen by using a closed contour in $\gamma$-plane around 0 or around 1 , and numerically integrating integer powers of $\gamma$ or $1-\gamma$ times the vertex. Based on Cauchy's formula - used here in reverse manner — one can then obtain a numerical evaluation of the residue of arbitrary order, and show that they actually vanish.
} 
in doing so one mixes different choices for $\mu_{R}$. But since $\alpha_{s}$ only varies by $\sim 4 \%$ between $35 \mathrm{GeV}$ and $50 \mathrm{GeV}$ we give the according result as well.

In ref. [35] it is argued based on ref. [36] that imposing $\left|\mathbf{k}_{J, 1}\right|>E,\left|\mathbf{k}_{J, 2}\right|>E+D$ for $D \rightarrow 0$ large logarithms of non-BFKL origin make a fixed order calculation unstable. Even though in a pure BFKL framework these logarithms do not show up at all, they - and their resummation - might be of significant impact on the result of a BFKL calculation. Therefore, $D \neq 0$ is preferred also in the BFKL framework to be safe from these unknown contributions. However, we start the presentation of our result with these symmetric scenarios but in order to be conservative and to avoid the region where initial state radiations might require peculiar treatment involving resummations à la Sudakov, which are beyond the scope of our work (and not implemented in fixed NLO calculation neither), we will close in section 4.3 with our results in the asymmetric case for which such resummations effects are clearly not required.

In all cases we choose the number of active flavors to be five $\left(N_{f}=5\right)$ with $\Lambda_{\mathrm{QCD}}=$ 221.2 MeV such that $\alpha_{s}\left(M_{Z}^{2}\right)=0.1176$.

The Monte Carlo integration [37] itself is error-prone. This error in Monte Carlo integration can be reduced to less than $1 \%$ with a large number of sample points, so as to be practically negligible in comparison with other sources of uncertainties. In practice, due to hardware/ time limitations we will display results for a Monte Carlo integration setup (for details see section A.2) which aims for an accuracy of the order of $1 \%$.

However, as we show in what follows, there are more serious uncertainties due to the renormalization scale $\mu_{R}$ which we choose as $\mu_{R}=\sqrt{\left|\mathbf{k}_{J, 1}\right| \cdot\left|\mathbf{k}_{J, 2}\right|}$. To study the dependence on it we vary $\mu_{R}$ by factors 2 and $1 / 2$ respectively. The same we do for the energy scale $\sqrt{s_{0}}$. We investigate the effect of the uncertainty of PDFs for asymmetric errors as defined in eqs. $(51,52)$ of $[16]$ with the eigenvector set ensuring all data sets being described within their $90 \%$ confidence level limits.

Below we present our results. The logic of their presentation is the following. For each kinematical situation, we discuss the physical observables and their uncertainties: crosssection encoded in $\mathcal{C}_{0}$, azimuthal decorrelation encoded in $\mathcal{C}_{1} / \mathcal{C}_{0}, \mathcal{C}_{2} / \mathcal{C}_{1}$ and $\mathcal{C}_{2} / \mathcal{C}_{1}$. We then give further details on the additional underlying quantities $\mathcal{C}_{1}, \mathcal{C}_{2}$. We use the same color coding for all plots, namely blue shows the pure LL result, brown the pure NLL result, green the combination of LL vertices with the collinear improved NLL Green's function, red the full NLL vertices with the collinear improved NLL Green's function. Whenever we show curves for scales $\mu_{R}=\mu_{F}$ or $s_{0}$ changed by factors 2 or $1 / 2$, the thick curve corresponds to the scale changed by factor 2 .

\section{$4.1 \quad\left|\mathbf{k}_{J, 1}\right|=\left|\mathbf{k}_{J, 2}\right|=35 \mathrm{GeV}$}

Thus the first thing to look at is the differential cross section as defined in eq. (2.8). The result of our calculation is shown in figure 3 (the according tabled values are shown in table 1 in the appendix). The decrease of the cross section at large values of $Y \gtrsim 7$ is mostly an effect of the upper kinematical cut on the rapidity of the single jets. This is true also for the coefficients $\mathcal{C}_{1}$ and $\mathcal{C}_{2}$ in figures 16 and 19 . 
The purely numerical error due to the Monte Carlo integration of the NLL vertices is mainly below $2 \%$ (see figure 5), and only for very large $Y$ of the order of $2-5 \%$. We varied the renormalization and factorization scale by factors 2 and $1 / 2$ to investigate the $\mu_{R}$ dependence. A full scan over this interval is not possible due to the CPU time consumption of the evaluation for a single choice of $\mu_{R}$. The results are displayed in figure 4 . As one would expect, the full NLL result depends less on $\mu_{R}$ than the LL result or the combination of LL vertices with resummed NLL Green's function which was so far stateof-the-art $[11,12]$. However, the results obtained for the three choices of $\mu_{R}$ studied here seem to suggest that, as expected, after inclusion of the NLL vertices the $\mu_{R}$ dependence is no longer monotone and flattens out, resulting in higher stability of NLL results. Another important scale is the energy $s_{0}$ introduced by the Mellin transformation from energy to $\omega$ space which is necessary to formulate the BFKL equation. Like $\mu_{R}$ it is an artificial scale which in an all order calculation would not affect the result. Indeed, the dependence is reduced when the NLL corrections to the vertices are taken into account (see figure 4).

The dependence on PDF uncertainties is shown in figure 5. This significant sensitivity is almost identical for pure LL, pure NLL, combined LL vertices with collinear improved NLL Green's function and for full NLL vertices combined with the collinear improved NLL Green's function.

The azimuthal decorrelation, described by coefficients defined in eq. (2.9), has often be predicted to be a striking feature of BFKL physics. Our results displayed in figure 6 for $\langle\cos \varphi\rangle$ and in figure 10 for $\langle\cos 2 \varphi\rangle$ explicitly show that our inclusion of NLL vertices leads to an enormous correlation in the azimuthal angle (for completeness our results for $\mathcal{C}_{1}$ and $\mathcal{C}_{2}$ coefficients alone are displayed respectively in figure 16 and figure 19 ).

In particular, $\langle\cos \varphi\rangle$ shown in figure 6 is rather close to the typical values predicted by LO-DGLAP Monte Carlos Pythia [38] and Herwig [39], used for CMS studies [15]. Note that Herwig has the tendency to predict more decorrelation, presumably because since it implements more radiations than PYтніA, it has the phenomenological effect to involve some kind of NLO-DGLAP corrections, which enhance the decorrelation. The various sources of uncertainty of our results are shown for $\langle\cos \varphi\rangle$ in figure 7 (variation of $\mu_{R}=\mu_{F}$ ), figure 8 (variation of $s_{0}$ ), and for $\langle\cos 2 \varphi\rangle$ in figure 11, and figure 12 accordingly.

Not only is the $Y$ dependence much flatter for the NLL vertices. But the mean value for $\cos \varphi$ itself is very close to 1 , especially for the NLL calculation including the collinear improved Green's function. Actually, in the collinear improved approach and for low values of $\mu_{R},\langle\cos \varphi\rangle=\mathcal{C}_{1} / \mathcal{C}_{0}$ can exceed unity. This feature has to be ascribed to the fact that the collinear improvement is justified and applied only to the $n=0$ conformal spin of the kernel, with the effect of lowering $\mathcal{C}_{0}$ in a large region of the phase space, while keeping $\mathcal{C}_{1}$ unchanged. For this reason, the predictions of angular dependent quantities are more trustable in the pure NLL approach (without collinear resummation).

The $\mu_{R}$ dependence of $\mathcal{C}_{1} / \mathcal{C}_{0}$ (see figure 7 ) is puzzling, but has to be considered as a consequence of the $\mathcal{C}_{0}$ and $\mathcal{C}_{1}$ dependencies in figures 4 and 17 respectively. A similar behavior can be observed for the $s_{0}$ dependence (see figure 8 for $\mathcal{C}_{1} / \mathcal{C}_{0}$ and figure 4 and figure 17 for $\mathcal{C}_{0}$ and $\mathcal{C}_{1}$ respectively). While the $\mu_{R}$ and $s_{0}$ dependences for $\mathcal{C}_{0}$ and $\mathcal{C}_{1}$ are significantly reduced by the inclusion of the NLL vertices, in the ratio this is only true if 
compared to the pure LL calculation. The combination of LL vertices and NLL collinear improved NLL Green's function is less sensitive on changes of $s_{0}$ or $\mu_{R}$. The reason for this surprising 'weakness' of the NLL result is that the changes of the LL vertices when changing $s_{0}$ or $\mu_{R}$ are not very sensitive on $n$ such that ratios of LL vertices are very stable against scale changes. In contrast, the NLL correction - especially the LL subtraction - is very large (and negative) for the $n=0$ component while of minor significance for $n>0$ such that effects of a scale change for NLL vertices do not vanish by considering ratios $\mathcal{C}_{n} / \mathcal{C}_{0}$. The special role of $n=0$ becomes apparent if one compares the situation to $\mathcal{C}_{n \neq 0} / \mathcal{C}_{m \neq 0}$ (see figures 15,14$)$ where the expected advantage of the full NLL calculation is clearly visible.

For both scales, the curves exceeding 1 belong to smaller scales (in the calculation with the collinear improved Green's function) which seem to be very disfavored in full NLL BFKL calculations as already discussed in [23, 40, 41]. The dependence on the PDFs completely drops out for LL vertices, and also for NLL vertices it is negligible as can be seen in figure 9.

A similar rather large dependency on $\mu_{R}=\mu_{F}$ and $s_{0}$ is obtained for $\mathcal{C}_{2} / \mathcal{C}_{0}$, although it does not lead to values of $\langle\cos 2 \varphi\rangle$ close to 1 , as can be seen from figures 11,12 , based on detailed studies of coefficients $\mathcal{C}_{0}$ and $\mathcal{C}_{2}$ displayed respectively in figures 4 and 20.

In ref. [11] it has been proposed to also study other observables $\mathcal{C}_{m} / \mathcal{C}_{n}$ with $m \neq 0 \neq n$. This is motivated by the fact that the main source of uncertainty of the Green's function is associated with the $n=0$ component. This observation is not altered by the inclusion of NLL vertices, as we display in figures 13-15. Moreover, figure 14 shows that the effect of changing $\mu_{R}=\mu_{F}$ leads to modifications of similar size for pure NLL and combined LL vertices with NLL Green's function predictions, while the changing of $s_{0}$ (see figure 15) leads to a reduced dependency with respect to $s_{0}$ of the pure NLL prediction. The PDF dependence for all ratios $\mathcal{C}_{m} / \mathcal{C}_{n}$ cancels in the same manner such that there is no use plotting it more than once (see figure 9 for $\mathcal{C}_{1} / \mathcal{C}_{0}$ ).

A priori, one would expect the numeric uncertainties for the $\mathcal{C}_{n>0}$ calculations including the NLL vertices to be larger because the coefficients $C_{n, \nu}$ contain an azimuthal integration which in the case of $n=0$ becomes trivial while for $n>0$ has to be carried out. This is indeed true for the NLL corrections alone, but since these corrections are more significant for $n=0$ than for $n>0$, in the sum together with the error-free LL vertices the opposite turns out to be true (the Monte Carlo errors are smaller than $1 \%$ for $\mathcal{C}_{1,2}$ as shown in figures 18 , and 21 respectively).

We would like to draw the reader's attention to the analogous effects for the kernel. For both the BFKL kernel and the Mueller Navelet vertices the NLL corrections to the $n=0$ component around $\nu=0$ are very large and negative while the relative corrections for $n>0$ are positive, much smaller than for $n=0$ and slowly increasing with $n$.

Finally, for other kinematical configurations to be discussed below, the same kind of PDF uncertainties appear, and Monte Carlo errors are of similar order. Because of that, we will not display the corresponding curves. 


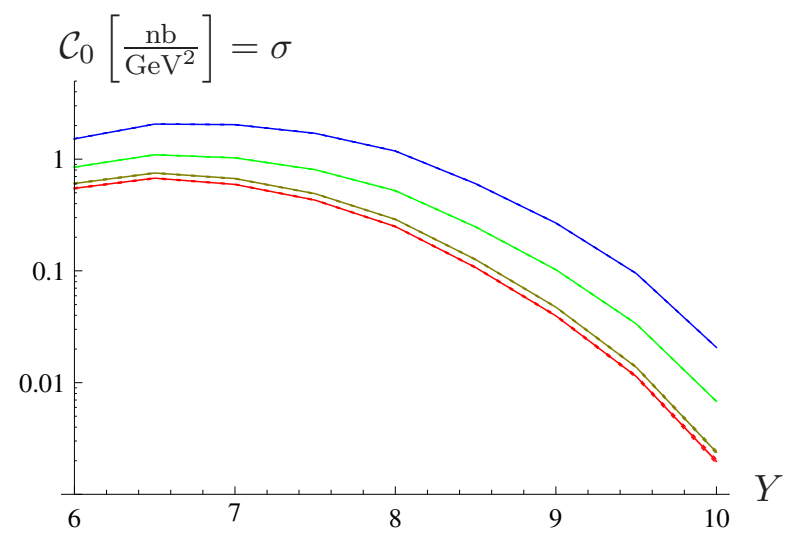

Figure 3. Differential cross section in dependence on $Y$ for $\left|\mathbf{k}_{J, 1}\right|=\left|\mathbf{k}_{J, 2}\right|=35 \mathrm{GeV}$. The errors due to the Monte Carlo integration - though hardly visible - are given as error bands. The tabled values are shown in table 1. Blue shows the pure LL result, brown the pure NLL result, green the combination of LL vertices with the collinear improved NLL Green's function, red the full NLL vertices with the collinear improved NLL Green's function. The same color coding is used in all subsequent plots.
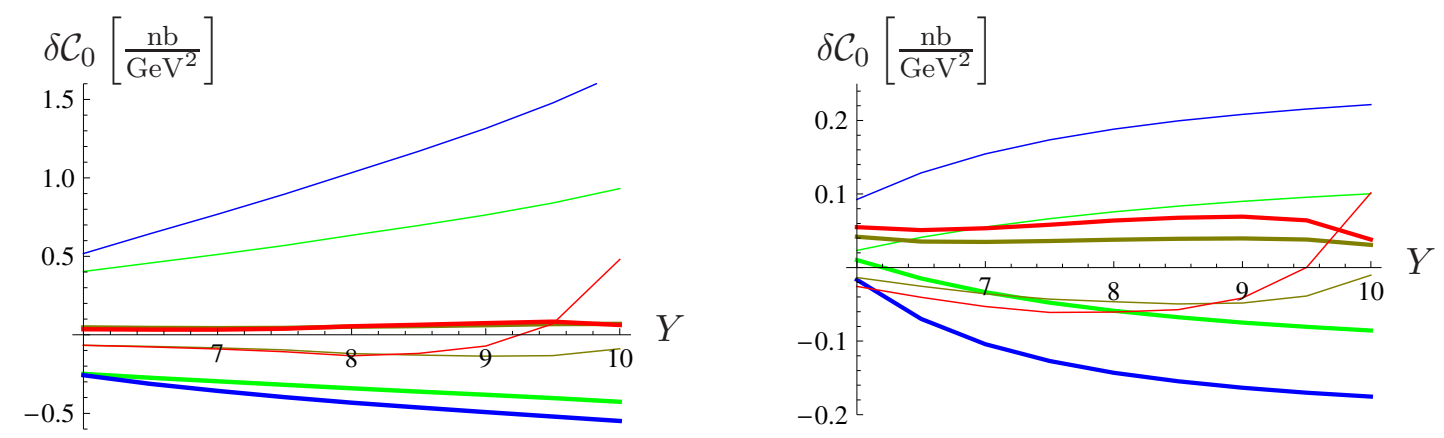

Figure 4. Relative effect of changing $\mu_{R}=\mu_{F}$ by factors 2 (thick) and $1 / 2$ (thin) respectively (left), and $\sqrt{s_{0}}$ (right) by factors 2 (thick) and $1 / 2$ (thin) resp. on the differential cross section in dependence on $Y$ for $\left|\mathbf{k}_{J, 1}\right|=\left|\mathbf{k}_{J, 2}\right|=35 \mathrm{GeV}$. The tabled values are shown in tables 2 and 3.
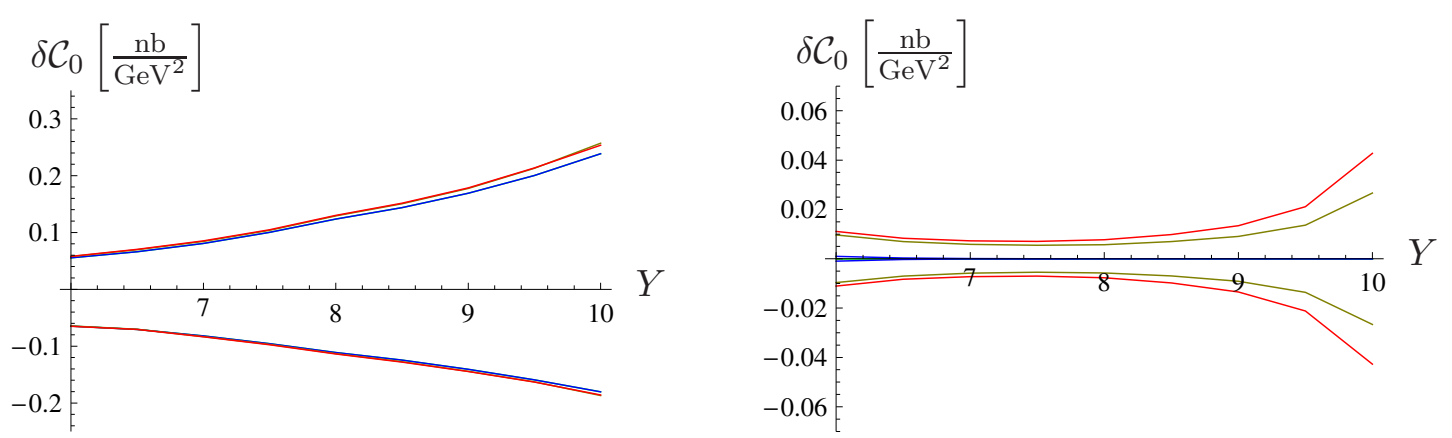

Figure 5. Relative effect of the PDF (left) and Monte Carlo (right) errors on the differential cross section in dependence on $Y$ for $\left|\mathbf{k}_{J, 1}\right|=\left|\mathbf{k}_{J, 2}\right|=35 \mathrm{GeV}$. The tabled values are shown in tables 4 and 1 . 


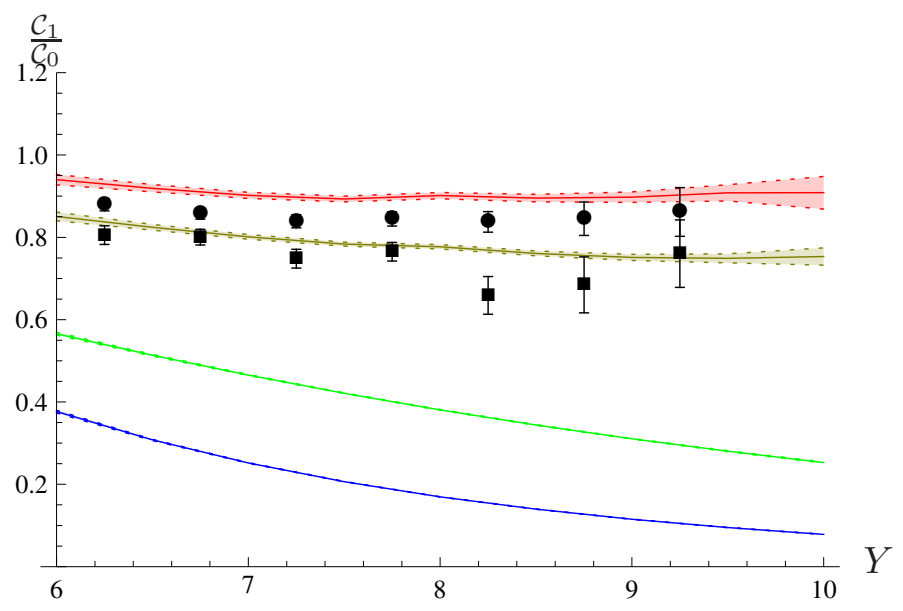

Figure 6. $\langle\cos \varphi\rangle$ in dependence on $Y$ for $\left|\mathbf{k}_{J, 1}\right|=\left|\mathbf{k}_{J, 2}\right|=35 \mathrm{GeV}$. The errors due to the Monte Carlo integration are given as error bands. The tabled values are shown in table 5. As dots are shown the results of ref. [15] obtained with Pyтhia [38]. As squares are shown the results of ref. [15] obtained with HERWIG [39].
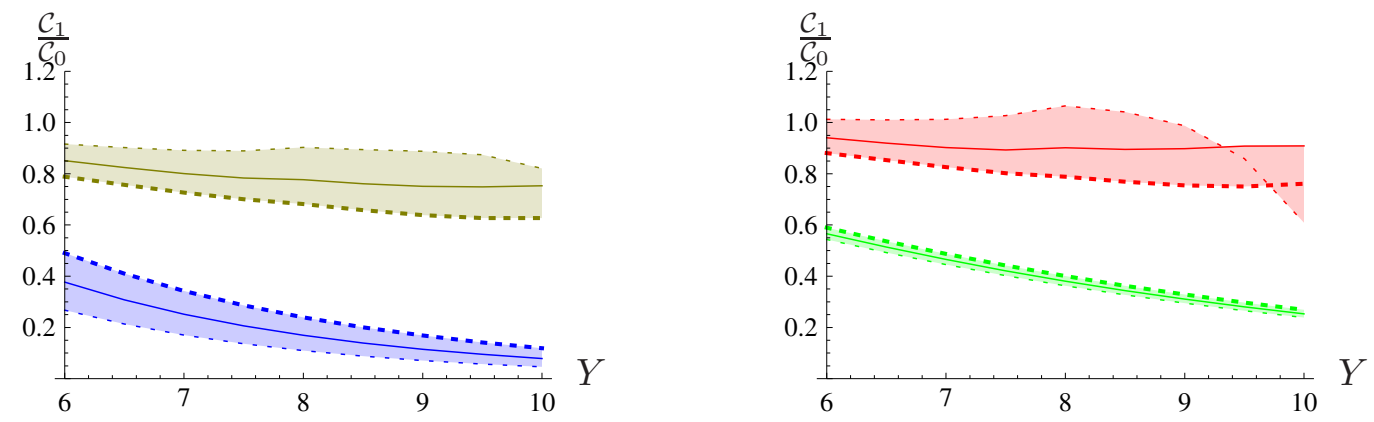

Figure 7. Effect of changing $\mu_{R}=\mu_{F}$ by factors 2 and $1 / 2$ respectively on $\langle\cos \varphi\rangle$ in dependence on $Y$ for $\left|\mathbf{k}_{J, 1}\right|=\left|\mathbf{k}_{J, 2}\right|=35 \mathrm{GeV}$. The tabled values are shown in table 6
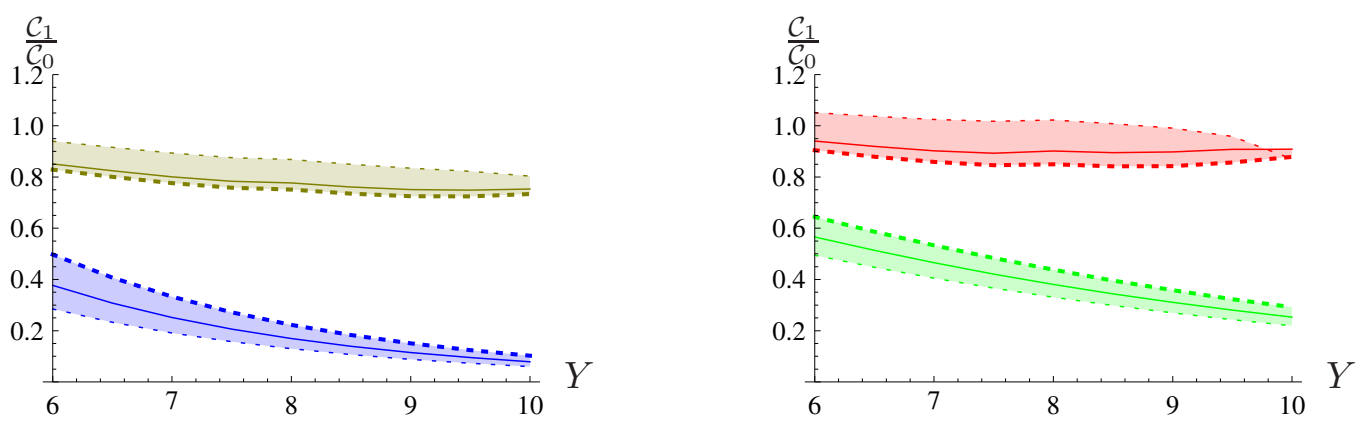

Figure 8. Effect of changing $\sqrt{s_{0}}$ by factors 2 and $1 / 2$ respectively on $\langle\cos \varphi\rangle$ in dependence on $Y$ for $\left|\mathbf{k}_{J, 1}\right|=\left|\mathbf{k}_{J, 2}\right|=35 \mathrm{GeV}$. The tabled values are shown in table 7 . 

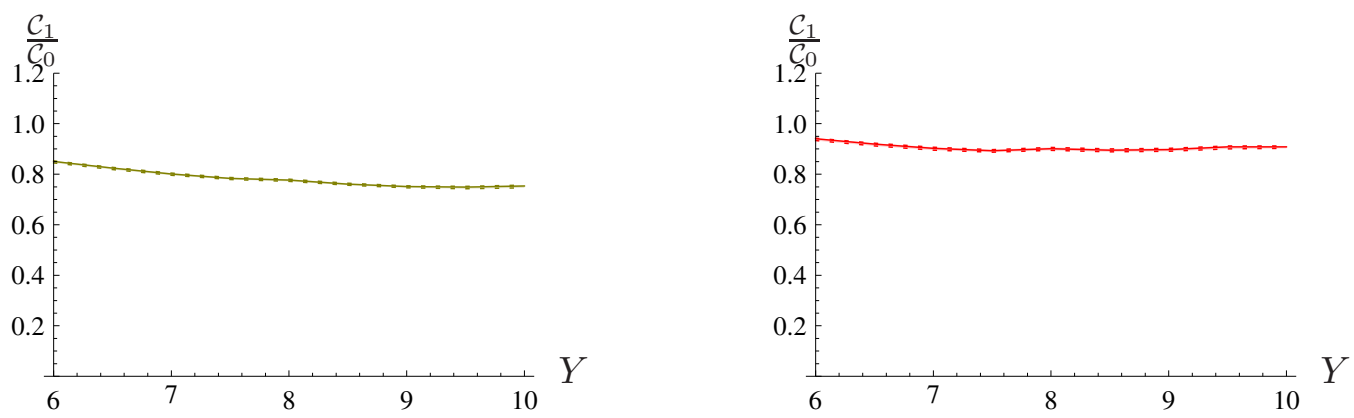

Figure 9. Effect of the PDF errors on $\langle\cos \varphi\rangle$ in dependence on $Y$ for $\left|\mathbf{k}_{J, 1}\right|=\left|\mathbf{k}_{J, 2}\right|=35 \mathrm{GeV}$. The tabled values are shown in table 8 .

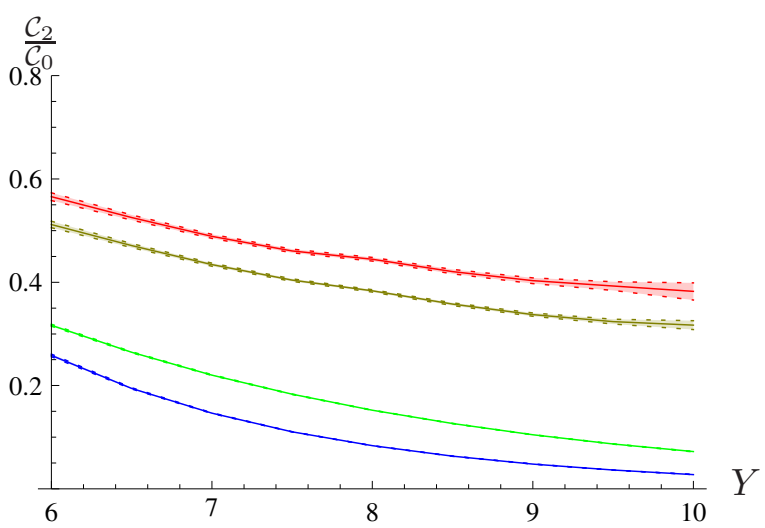

Figure 10. $\langle\cos 2 \varphi\rangle$ in dependence on $Y$ for $\left|\mathbf{k}_{J, 1}\right|=\left|\mathbf{k}_{J, 2}\right|=35 \mathrm{GeV}$. The errors due to the Monte Carlo integration are given as error bands. The tabled values are shown in table 9.
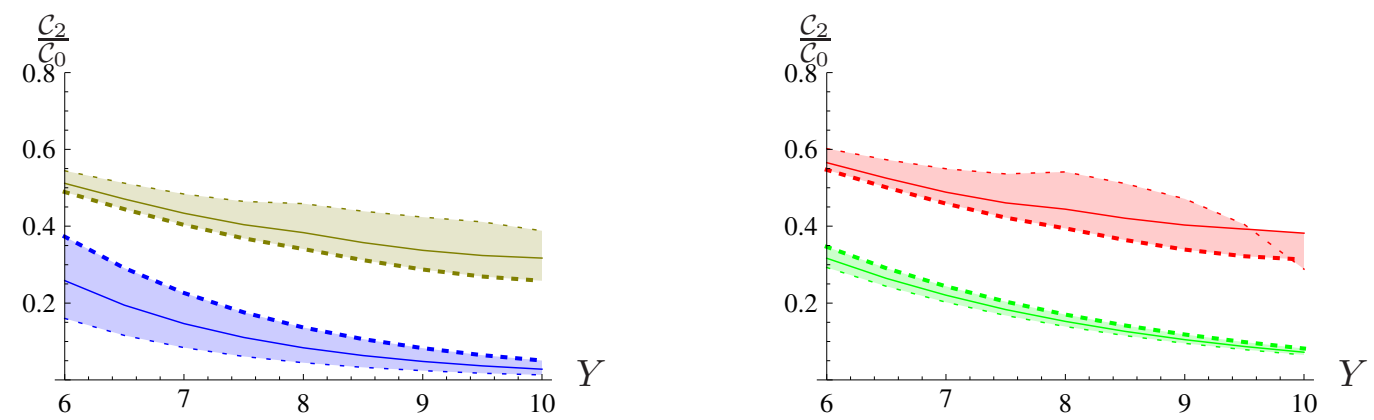

Figure 11. Effect of changing $\mu_{R}=\mu_{F}$ by factors 2 and $1 / 2$ respectively on $\langle\cos 2 \varphi\rangle$ in dependence on $Y$ for $\left|\mathbf{k}_{J, 1}\right|=\left|\mathbf{k}_{J, 2}\right|=35 \mathrm{GeV}$. The tabled values are shown in table 10 . 

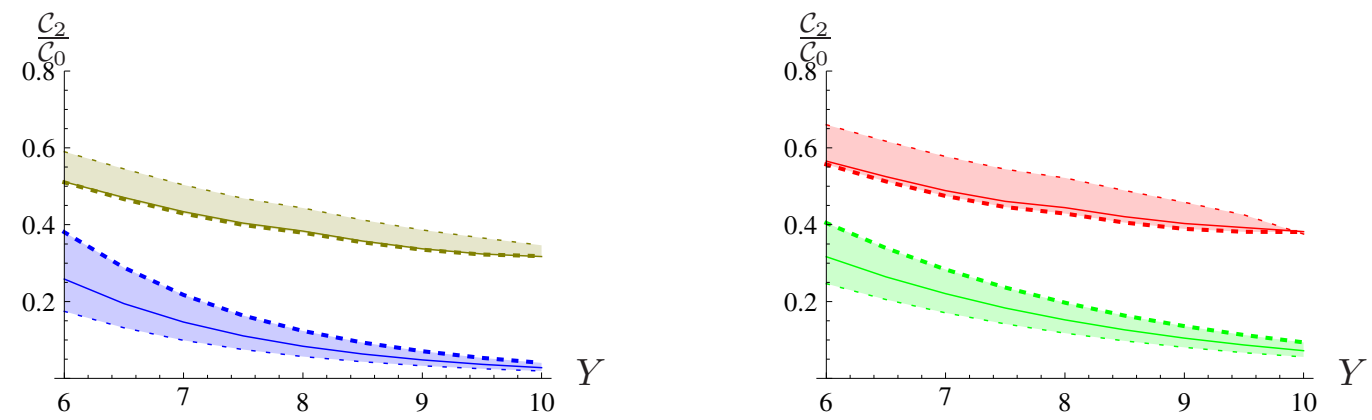

Figure 12. Effect of changing $\sqrt{s_{0}}$ by factors 2 and $1 / 2$ respectively on $\langle\cos 2 \varphi\rangle$ in dependence on $Y$ for $\left|\mathbf{k}_{J, 1}\right|=\left|\mathbf{k}_{J, 2}\right|=35 \mathrm{GeV}$. The tabled values are shown in table 11 .

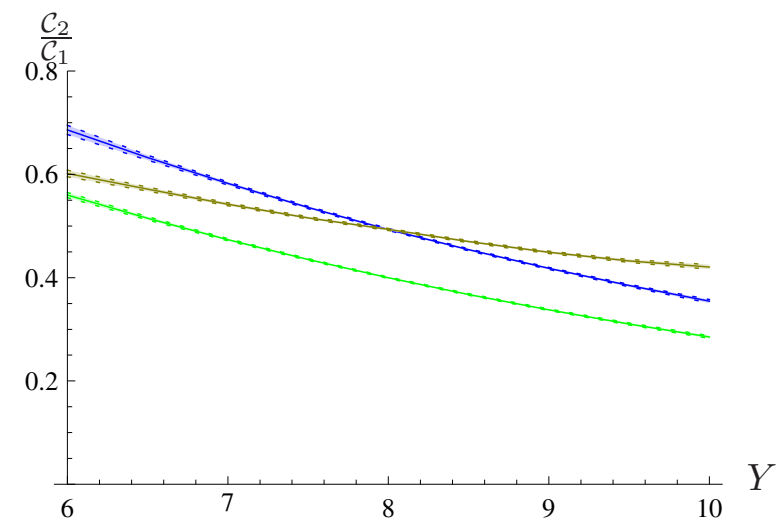

Figure 13. $\langle\cos 2 \varphi\rangle /\langle\cos \varphi\rangle$ in dependence on $Y$ for $\left|\mathbf{k}_{J, 1}\right|=\left|\mathbf{k}_{J, 2}\right|=35 \mathrm{GeV}$. The errors due to the Monte Carlo integration — though hardly visible - are given as error bands. The tabled values are shown in table 12 .
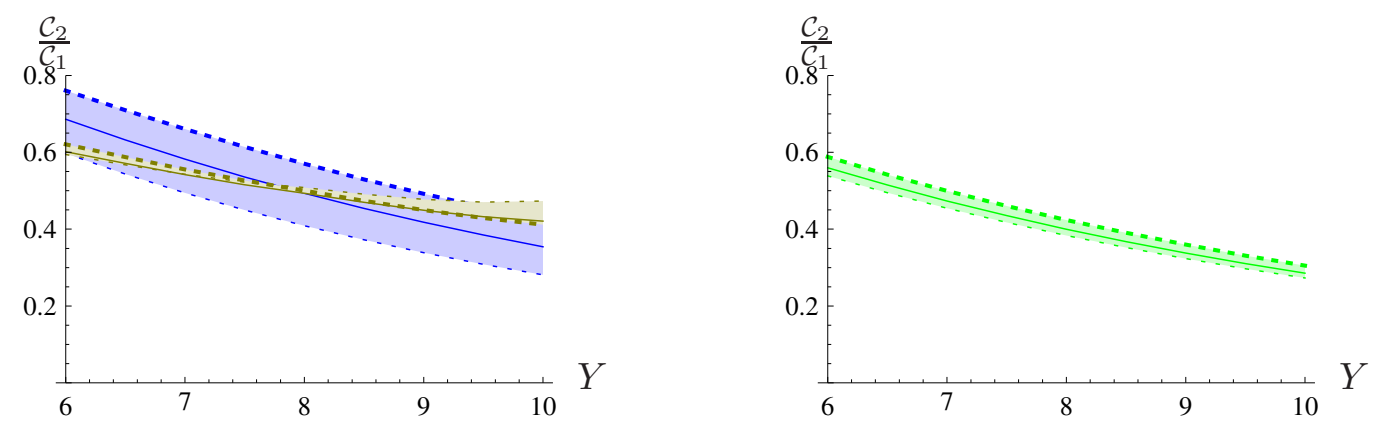

Figure 14. Effect of changing $\mu_{R}=\mu_{F}$ by factors 2 and $1 / 2$ respectively on $\langle\cos 2 \varphi\rangle /\langle\cos \varphi\rangle$ in dependence on $Y$ for $\left|\mathbf{k}_{J, 1}\right|=\left|\mathbf{k}_{J, 2}\right|=35 \mathrm{GeV}$. The tabled values are shown in table 13 . 

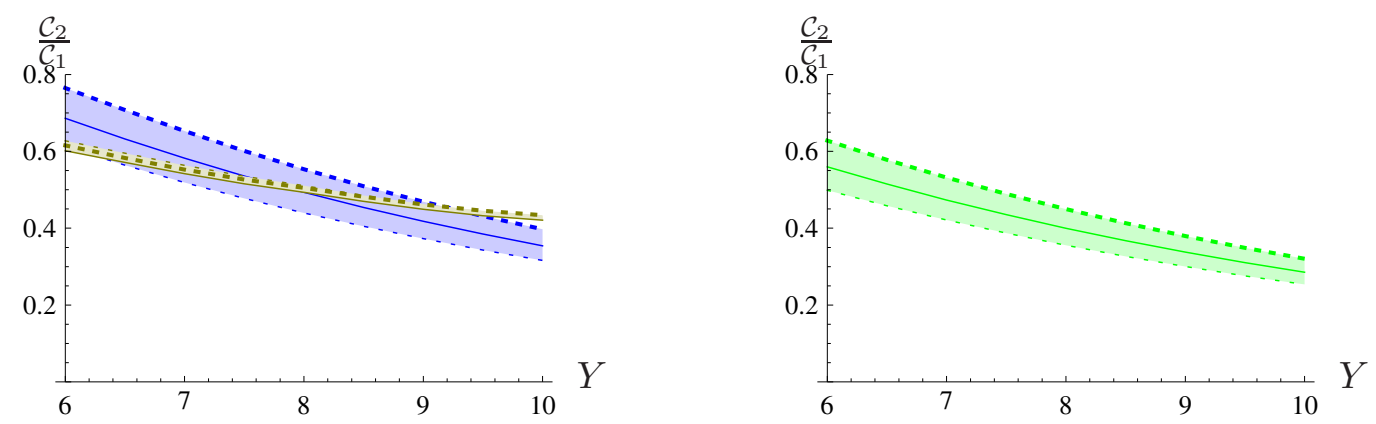

Figure 15. Effect of changing $\sqrt{s_{0}}$ by factors 2 and $1 / 2$ respectively on $\langle\cos 2 \varphi\rangle /\langle\cos \varphi\rangle$ in dependence on $Y$ for $\left|\mathbf{k}_{J, 1}\right|=\left|\mathbf{k}_{J, 2}\right|=35 \mathrm{GeV}$. The tabled values are shown in table 14 .

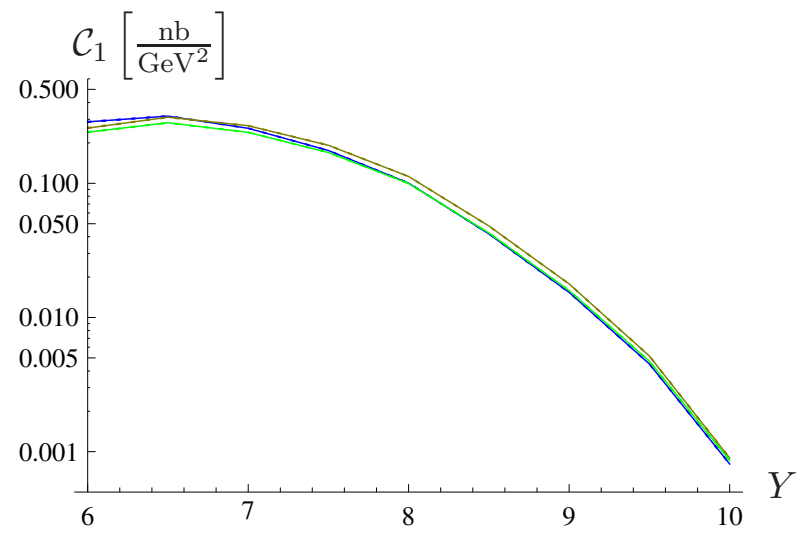

Figure 16. Coefficient $\mathcal{C}_{1}$ in dependence on $Y$ for $\left|\mathbf{k}_{J, 1}\right|=\left|\mathbf{k}_{J, 2}\right|=35 \mathrm{GeV}$. The errors due to the Monte Carlo integration — though hardly visible — are given as error bands. The tabled values are shown in table 15 .
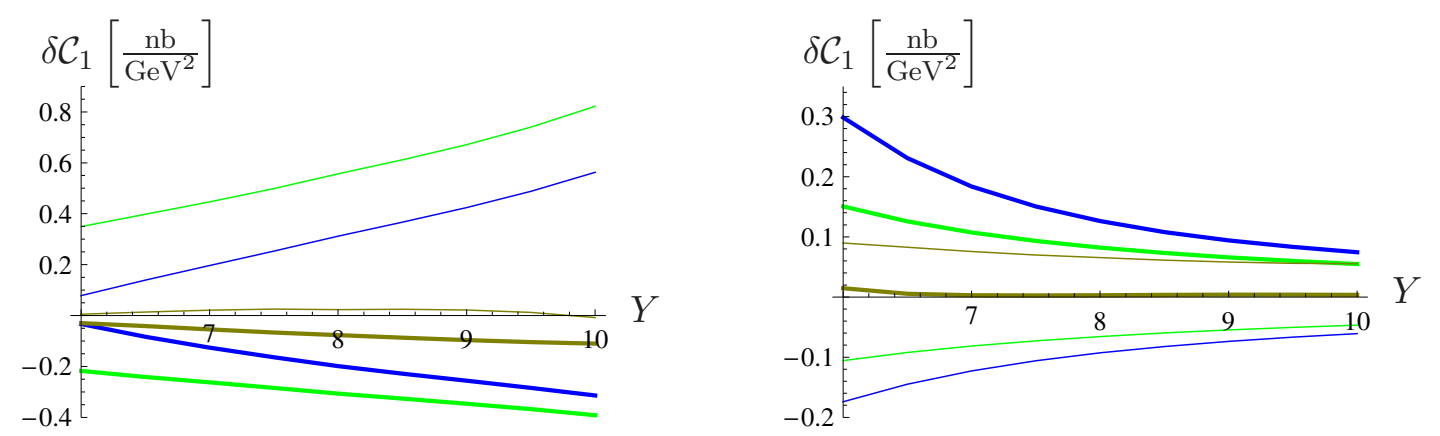

Figure 17. Relative effect of changing $\mu_{R}=\mu_{F}$ by factors 2 and $1 / 2$ respectively (left), and $\sqrt{s_{0}}$ (right) by factors 2 and $1 / 2$ respectively on the coefficient $\mathcal{C}_{1}$ in dependence on $Y$ for $\left|\mathbf{k}_{J, 1}\right|=$ $\left|\mathbf{k}_{J, 2}\right|=35 \mathrm{GeV}$. The tabled values are shown in tables 16 and 17 . 

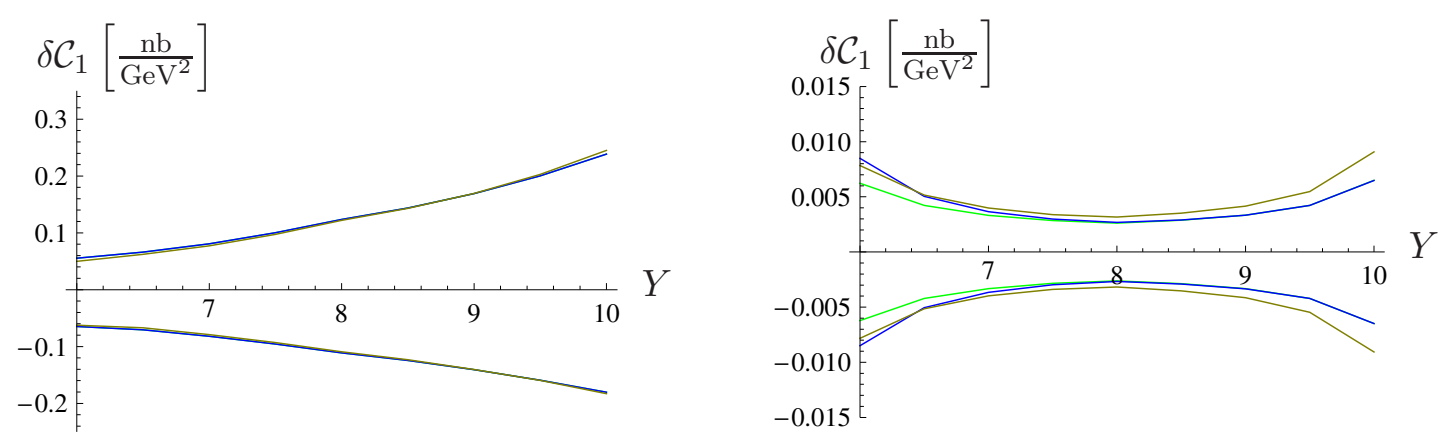

Figure 18. Relative effect of the PDF (left) and Monte Carlo (right) errors on the coefficient $\mathcal{C}_{1}$ in dependence on $Y$ for $\left|\mathbf{k}_{J, 1}\right|=\left|\mathbf{k}_{J, 2}\right|=35 \mathrm{GeV}$. The tabled values are shown in tables 18 and 15 .

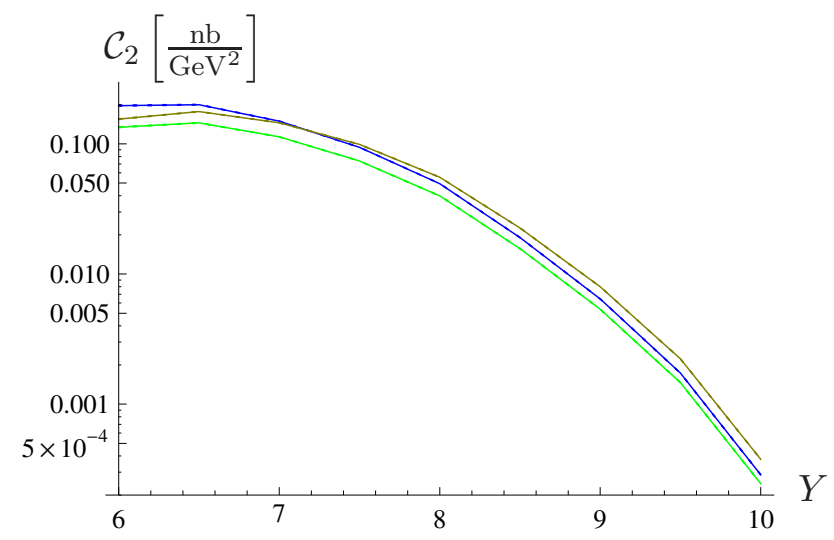

Figure 19. Coefficient $\mathcal{C}_{2}$ in dependence on $Y$ for $\left|\mathbf{k}_{J, 1}\right|=\left|\mathbf{k}_{J, 2}\right|=35 \mathrm{GeV}$. The errors due to the Monte Carlo integration - though hardly visible - are given as error bands. The tabled values are shown in table 19 .
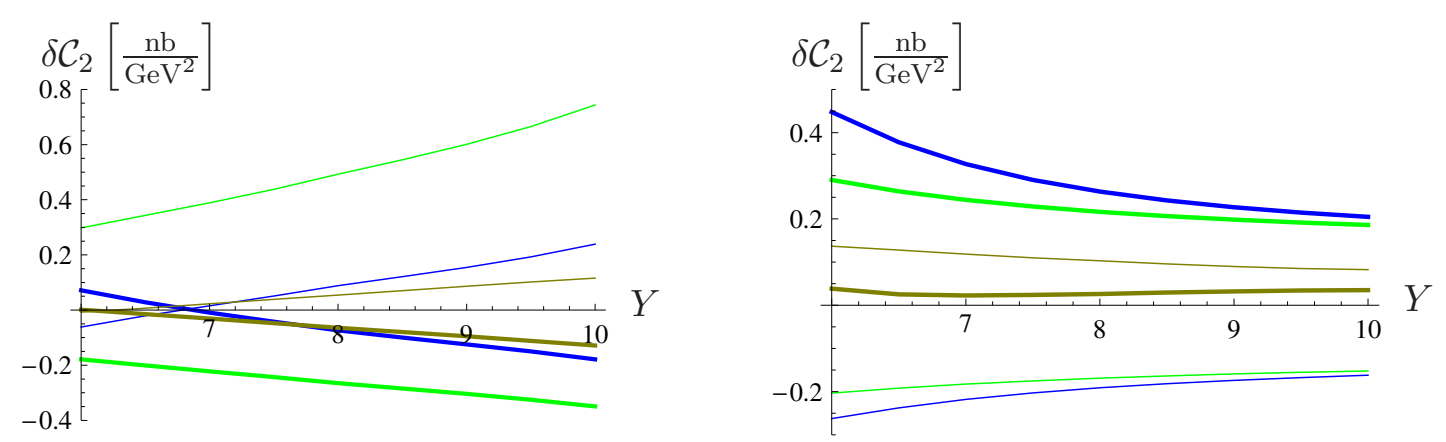

Figure 20. Relative effect of changing $\mu_{R}=\mu_{F}$ by factors 2 and $1 / 2$ respectively (left), and $\sqrt{s_{0}}$ (right) by factors 2 and $1 / 2$ respectively on the coefficient $\mathcal{C}_{2}$ in dependence on $Y$ for $\left|\mathbf{k}_{J, 1}\right|=$ $\left|\mathbf{k}_{J, 2}\right|=35 \mathrm{GeV}$. The tabled values are shown in tables 20 and 21 . 

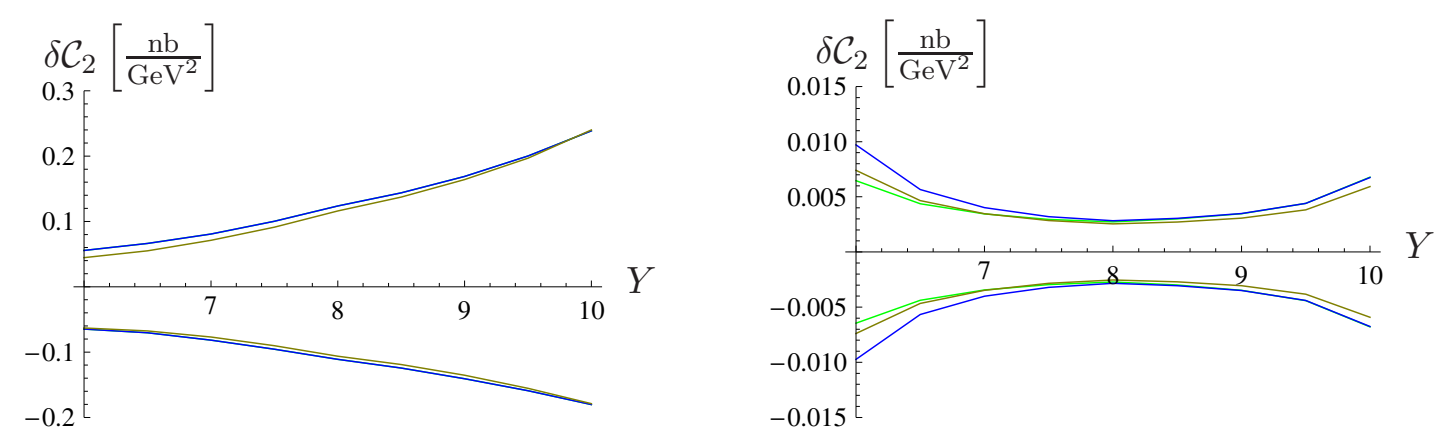

Figure 21. Relative effect of the PDF (left) and Monte Carlo (right) errors on the coefficient $\mathcal{C}_{2}$ in dependence on $Y$ for $\left|\mathbf{k}_{J, 1}\right|=\left|\mathbf{k}_{J, 2}\right|=35 \mathrm{GeV}$. The tabled values are shown in tables 22 and 19.

\section{$4.2\left|\mathbf{k}_{J, 1}\right|=\left|\mathbf{k}_{J, 2}\right|=50 \mathrm{GeV}$}

Going to larger jet scales, we meet more or less the same advantages and problems as for $35 \mathrm{GeV}$. Again we start with the differential cross section (2.8). The result is shown in figure 22 (the according tabled values are shown in table 23 in the appendix). The dependences with respect to $\mu_{R}$, and $s_{0}$ are displayed in figure 23 .

The azimuthal decorrelation is displayed in figure 24 for $\langle\cos \varphi\rangle$ and in figure 27 for $\langle\cos 2 \varphi\rangle$, again explicitly showing that inclusion of NLL vertices leads to an enormous correlation in the azimuthal angle (for completeness our results for $\mathcal{C}_{1}$ and $\mathcal{C}_{2}$ coefficients alone are displayed respectively in figure 33 and figure 35). Here, the angular correlation even has the tendency to increase with growing rapidity $Y$. This might be interpreted as the effect of stronger limited phase space for additional emissions at large energies and large transverse momenta of the produced jets (Note, that the cross section is a factor $\sim 10$ smaller at $Y=6$ compared to the previous configuration, and a factor $\sim 100$ smaller at $Y=10)$.

The various sources of uncertainty of our results are shown for $\langle\cos \varphi\rangle$ in figure 25 (variation of $\mu_{R}=\mu_{F}$ ), figure 26 (variation of $s_{0}$ ), and for $\langle\cos 2 \varphi\rangle$ in figure 28 (variation of $\mu_{R}=\mu_{F}$ ), figure 29 (variation of $s_{0}$ ).

The scale dependences of $\mathcal{C}_{1} / \mathcal{C}_{0}$ (see figures 25 , and 26) as well as of $\mathcal{C}_{0}$ (see figure 23) and $\mathcal{C}_{1}$ (see figure 34 ) alone reveal the same basic features as before, namely a non-monotone scale dependence of the NLL corrections and (more serious) unphysical results for $\langle\cos \varphi\rangle$ in case of the resummed NLL prediction for small $s_{0}$ and/ or $\mu_{R}=\mu_{F}$ scales.

A similar rather large dependency on $\mu_{R}=\mu_{F}$ and $s_{0}$ is obtained for $\mathcal{C}_{2} / \mathcal{C}_{0}$, as can be seen from figures 28, 29, based on detailed studies of coefficients $\mathcal{C}_{0}$ and $\mathcal{C}_{2}$ displayed respectively in figure 23 and figure 36 .

The problematic behavior for smaller scales of $s_{0}$ and/ or $\mu_{R}$ is more dramatic for $\left|\mathbf{k}_{J, 1}\right|=\left|\mathbf{k}_{J, 2}\right|=50 \mathrm{GeV}$ (see e.g figures 25, 26, 28, 29). Especially the $\mu_{R}$ dependence (see figure 25 seems to indicate that already the a priori natural scale $\mu_{R}=\left|\mathbf{k}_{J}\right|$ is too small. 


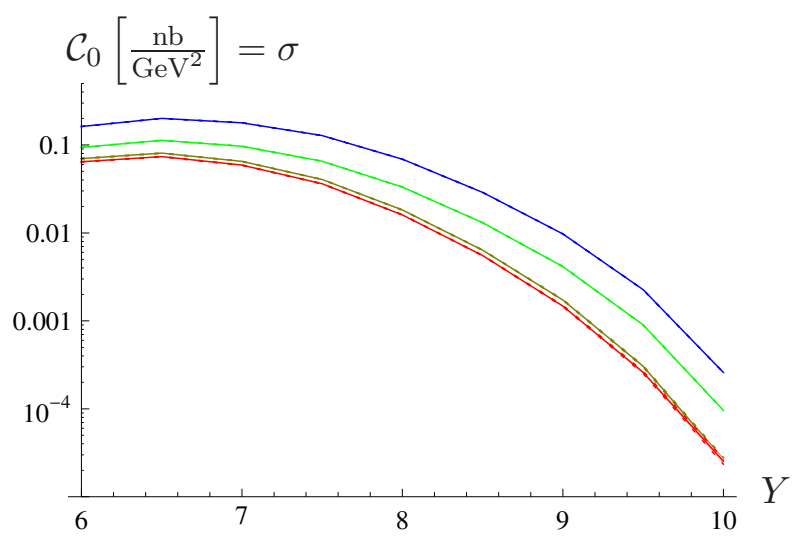

Figure 22. Differential cross section in dependence on $Y$ for $\left|\mathbf{k}_{J, 1}\right|=\left|\mathbf{k}_{J, 2}\right|=50 \mathrm{GeV}$. The errors due to the Monte Carlo integration - though hardly visible - are given as error bands. The tabled values are shown in table 23.
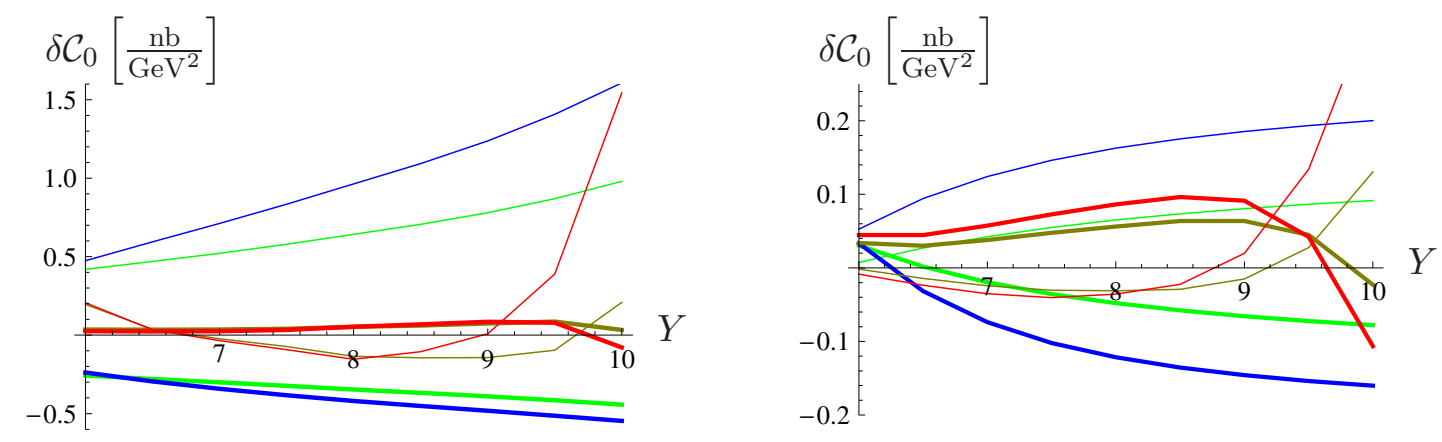

Figure 23. Relative effect of changing $\mu_{R}=\mu_{F}$ by factors 2 and $1 / 2$ respectively (left), and $\sqrt{s_{0}}$ (right) by factors 2 and $1 / 2$ respectively on the differential cross section in dependence on $Y$ for $\left|\mathbf{k}_{J, 1}\right|=\left|\mathbf{k}_{J, 2}\right|=50 \mathrm{GeV}$. The tabled values are shown in tables 24 and 25 .

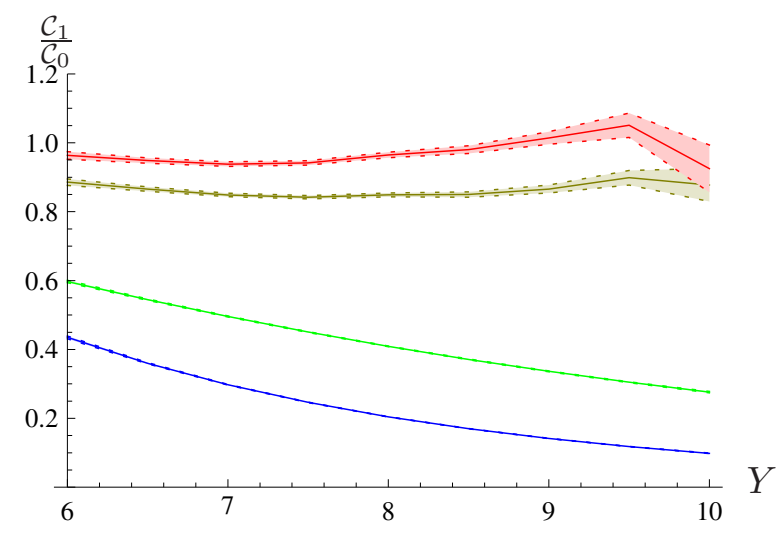

Figure 24. $\langle\cos \varphi\rangle$ in dependence on $Y$ for $\left|\mathbf{k}_{J, 1}\right|=\left|\mathbf{k}_{J, 2}\right|=50 \mathrm{GeV}$. The errors due to the Monte Carlo integration are given as error bands. The tabled values are shown in table 26. 

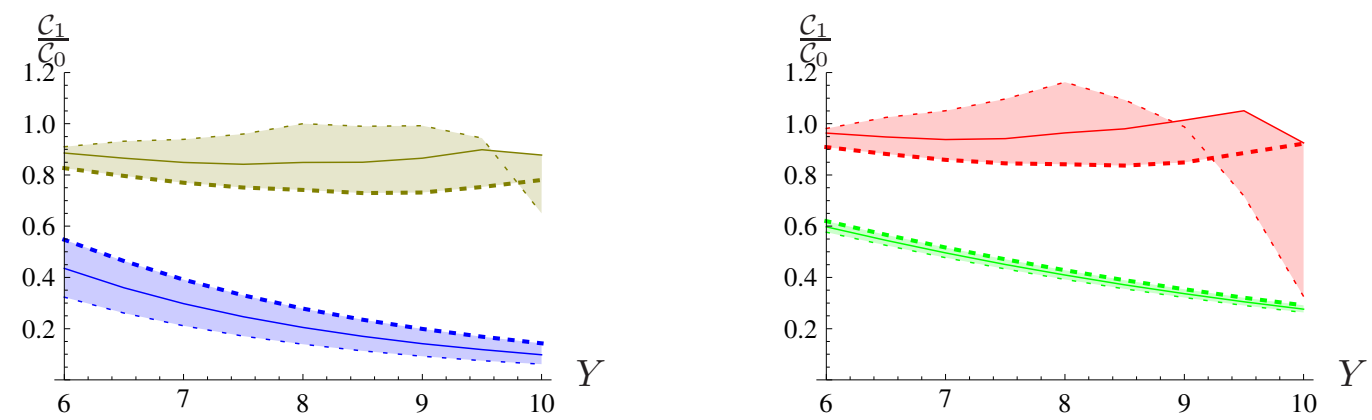

Figure 25. Effect of changing $\mu_{R}=\mu_{F}$ by factors 2 and $1 / 2$ respectively on $\langle\cos \varphi\rangle$ in dependence on $Y$ for $\left|\mathbf{k}_{J, 1}\right|=\left|\mathbf{k}_{J, 2}\right|=50 \mathrm{GeV}$. The tabled values are shown in table 27 .
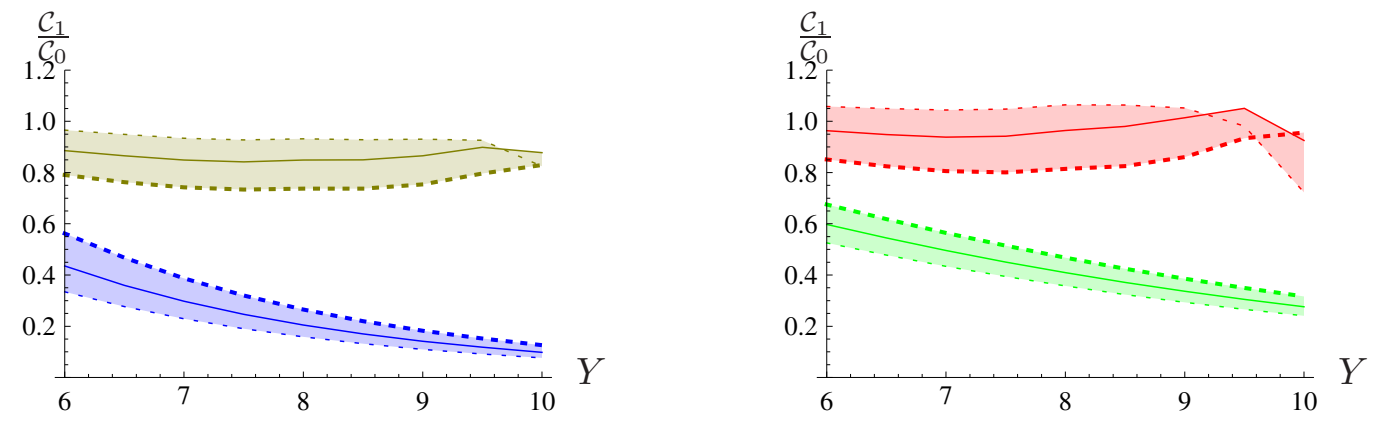

Figure 26. Effect of changing $\sqrt{s_{0}}$ by factors 2 and $1 / 2$ respectively on $\langle\cos \varphi\rangle$ in dependence on $Y$ for $\left|\mathbf{k}_{J, 1}\right|=\left|\mathbf{k}_{J, 2}\right|=50 \mathrm{GeV}$. The tabled values are shown in table 28 .

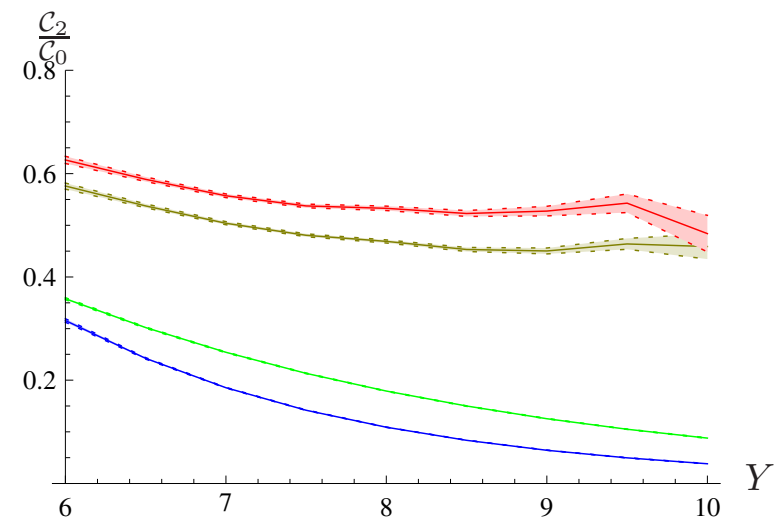

Figure 27. $\langle\cos 2 \varphi\rangle$ in dependence on $Y$ for $\left|\mathbf{k}_{J, 1}\right|=\left|\mathbf{k}_{J, 2}\right|=50 \mathrm{GeV}$. The errors due to the Monte Carlo integration are given as error bands. The tabled values are shown in table 29. 

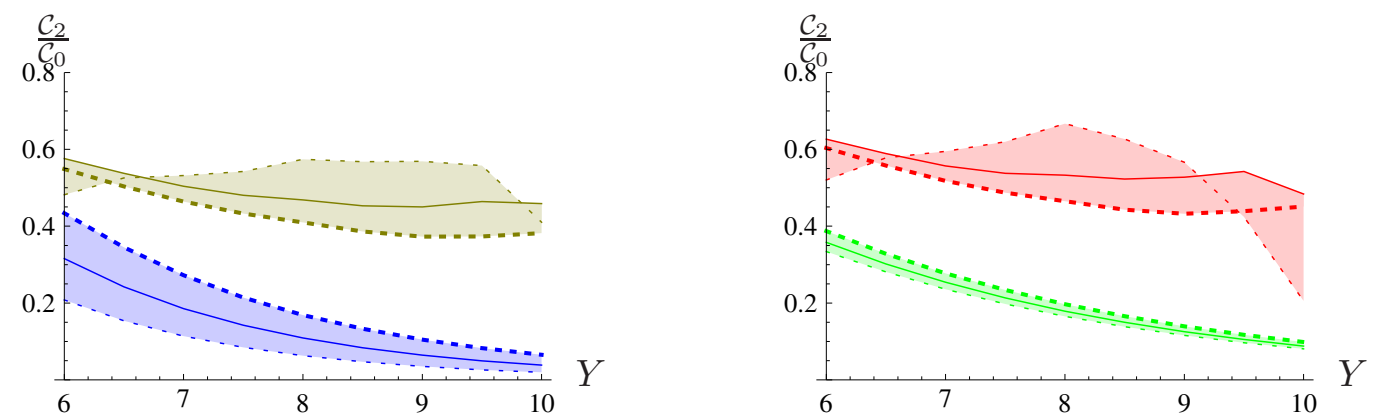

Figure 28. Effect of changing $\mu_{R}=\mu_{F}$ by factors 2 and $1 / 2$ respectively on $\langle\cos 2 \varphi\rangle$ in dependence on $Y$ for $\left|\mathbf{k}_{J, 1}\right|=\left|\mathbf{k}_{J, 2}\right|=50 \mathrm{GeV}$. The tabled values are shown in table 30 .
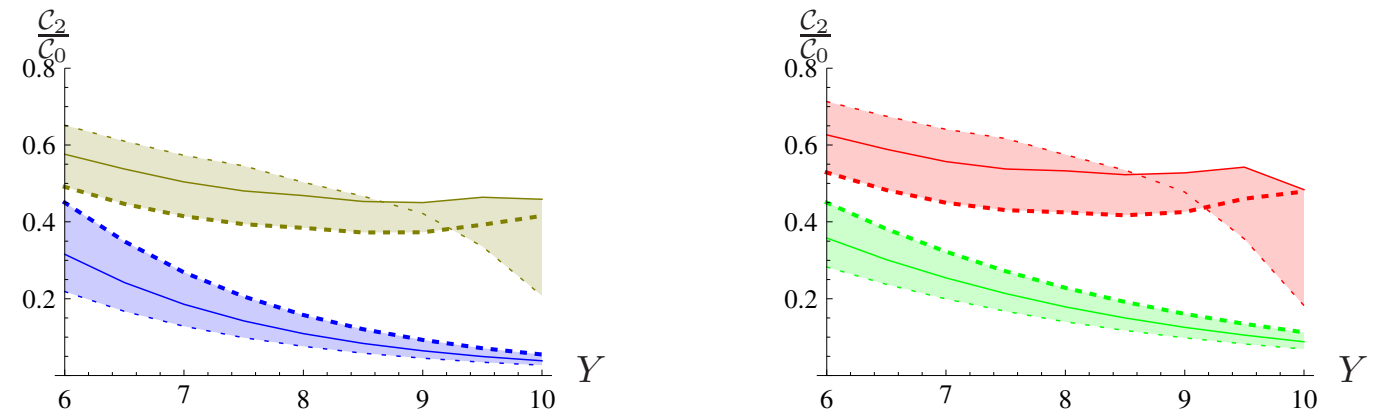

Figure 29. Effect of changing $\sqrt{s_{0}}$ by factors 2 and $1 / 2$ respectively on $\langle\cos 2 \varphi\rangle$ in dependence on $Y$ for $\left|\mathbf{k}_{J, 1}\right|=\left|\mathbf{k}_{J, 2}\right|=50 \mathrm{GeV}$. The tabled values are shown in table 31 .

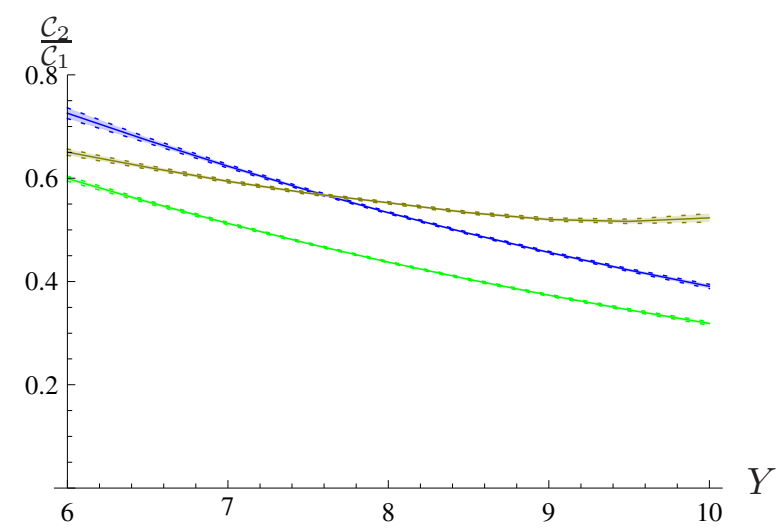

Figure 30. $\langle\cos 2 \varphi\rangle /\langle\cos \varphi\rangle$ in dependence on $Y$ for $\left|\mathbf{k}_{J, 1}\right|=\left|\mathbf{k}_{J, 2}\right|=50 \mathrm{GeV}$. The errors due to the Monte Carlo integration - though hardly visible - are given as error bands. The tabled values are shown in table 32 .

\section{$4.3\left|\mathbf{k}_{J, 1}\right|=35 \mathrm{GeV},\left|\mathbf{k}_{J, 2}\right|=50 \mathrm{GeV}$}

We end up with the consideration of the asymmetric case, which we investigate in order to provide a comparison with NLO-DGLAP predictions [42] obtained through the NLO- 

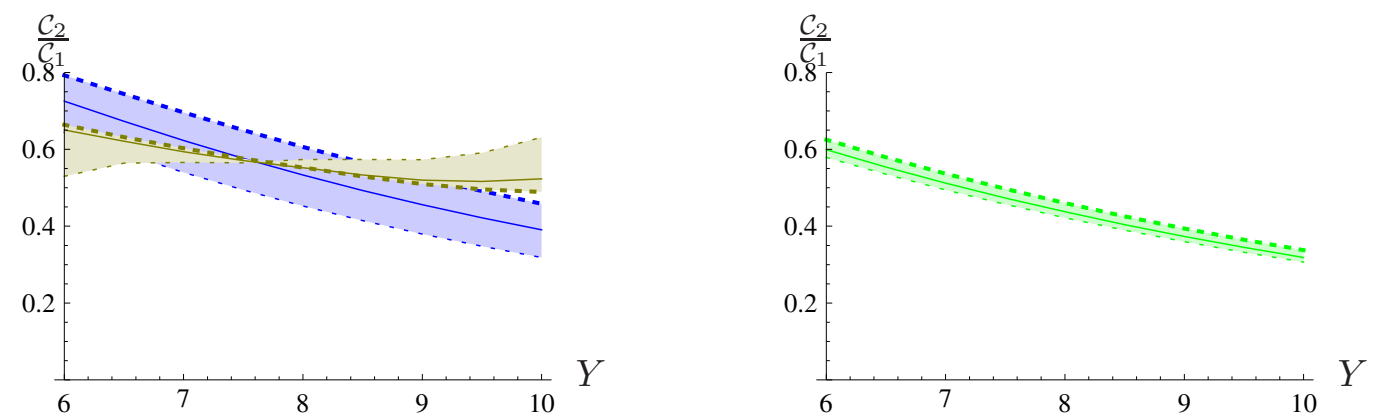

Figure 31. Effect of changing $\mu_{R}=\mu_{F}$ by factors 2 and $1 / 2$ respectively on $\langle\cos 2 \varphi\rangle /\langle\cos \varphi\rangle$ in dependence on $Y$ for $\left|\mathbf{k}_{J, 1}\right|=\left|\mathbf{k}_{J, 2}\right|=50 \mathrm{GeV}$. The tabled values are shown in table 33 .
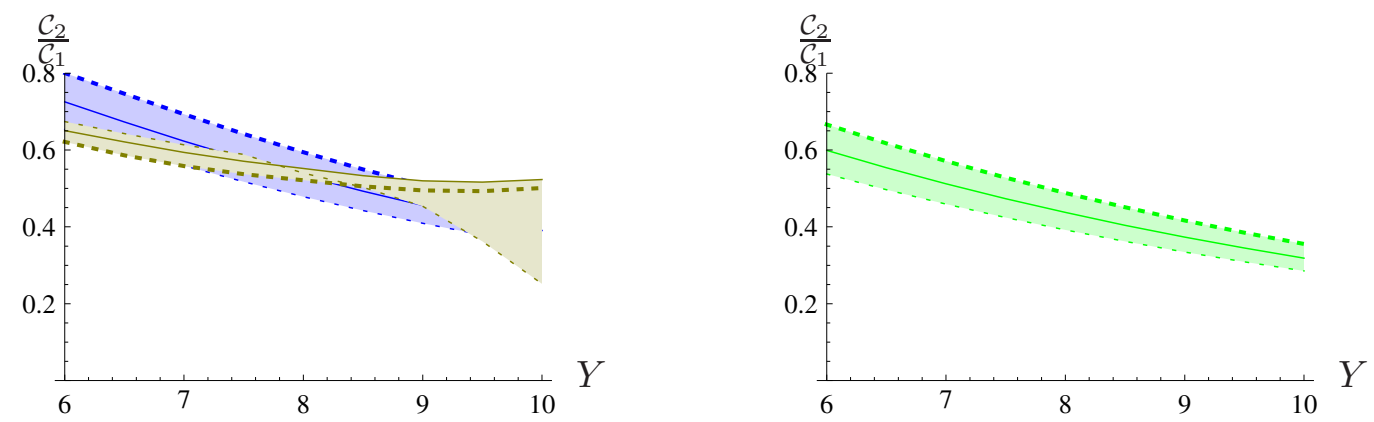

Figure 32. Effect of changing $\sqrt{s_{0}}$ by factors 2 and $1 / 2$ respectively on $\langle\cos 2 \varphi\rangle /\langle\cos \varphi\rangle$ in dependence on $Y$ for $\left|\mathbf{k}_{J, 1}\right|=\left|\mathbf{k}_{J, 2}\right|=50 \mathrm{GeV}$. The tabled values are shown in table 34 .

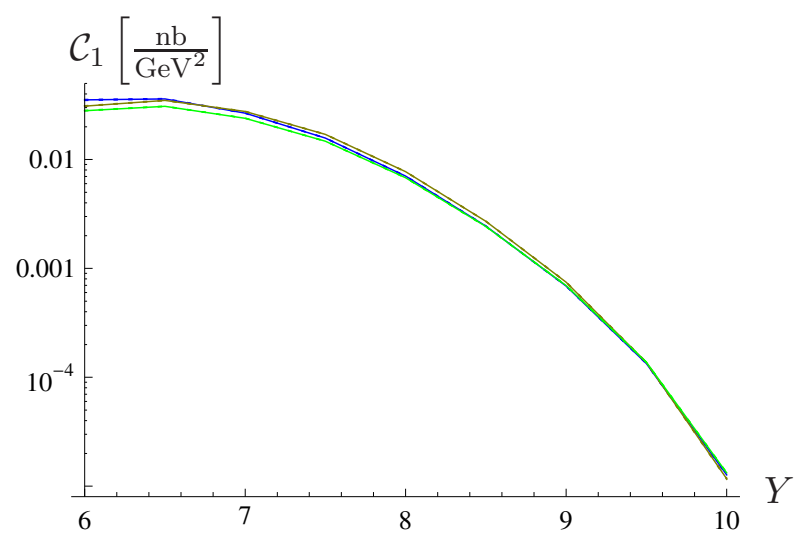

Figure 33. Coefficient $\mathcal{C}_{1}$ in dependence on $Y$ for $\left|\mathbf{k}_{J, 1}\right|=\left|\mathbf{k}_{J, 2}\right|=50 \mathrm{GeV}$. The errors due to the Monte Carlo integration - though hardly visible - are given as error bands. The tabled values are shown in table 35 .

DGLAP partonic generator DIJET [43]. These prediction are very sensitive to the precise compensation between the real and the virtual contribution, and a symmetric cut leads to 

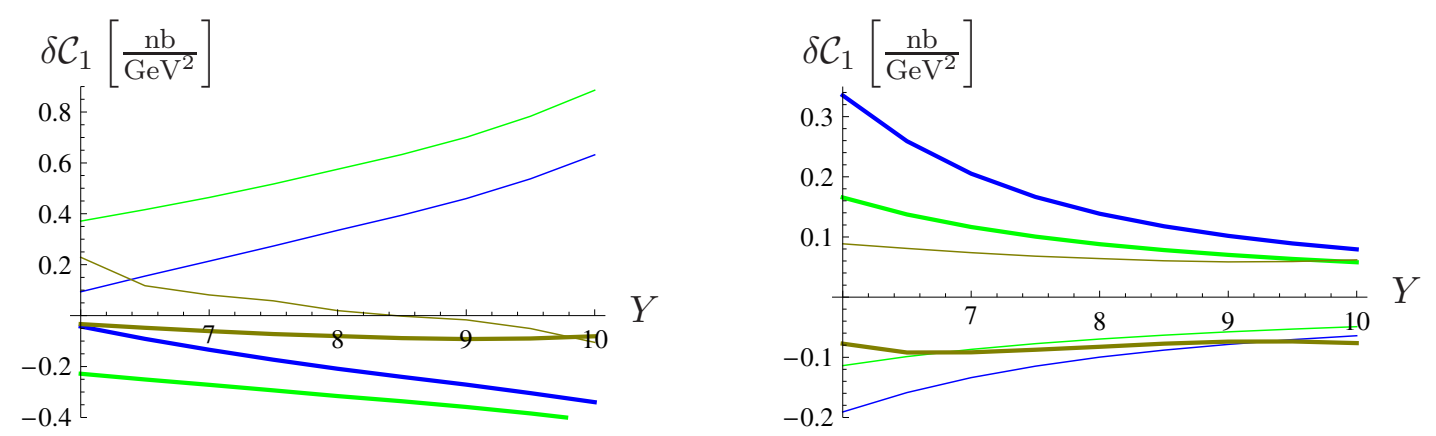

Figure 34. Relative effect of changing $\mu_{R}=\mu_{F}$ by factors 2 and $1 / 2$ respectively (left), and $\sqrt{s_{0}}$ (right) by factors 2 and $1 / 2$ respectively on the coefficient $\mathcal{C}_{1}$ in dependence on $Y$ for $\left|\mathbf{k}_{J, 1}\right|=$ $\left|\mathbf{k}_{J, 2}\right|=50 \mathrm{GeV}$. The tabled values are shown in tables 36 and 37 .

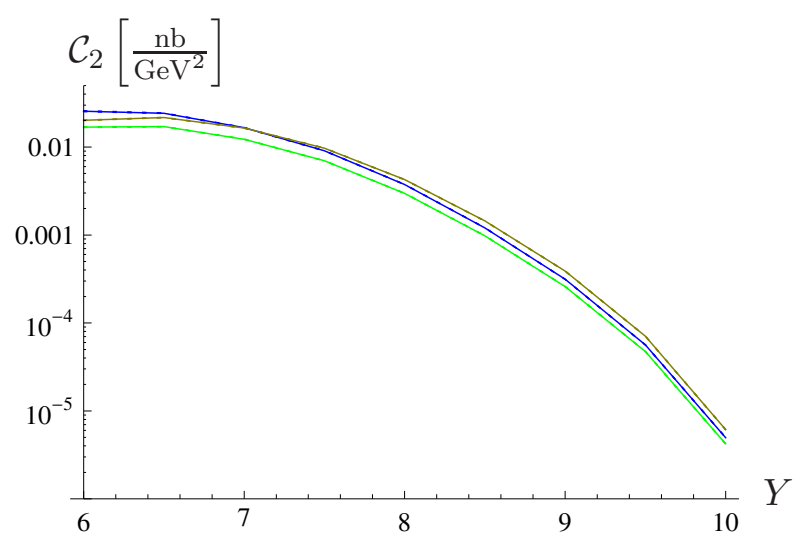

Figure 35. Coefficient $\mathcal{C}_{2}$ in dependence on $Y$ for $\left|\mathbf{k}_{J, 1}\right|=\left|\mathbf{k}_{J, 2}\right|=50 \mathrm{GeV}$. The errors due to the Monte Carlo integration - though hardly visible - are given as error bands. The tabled values are shown in table 38 .
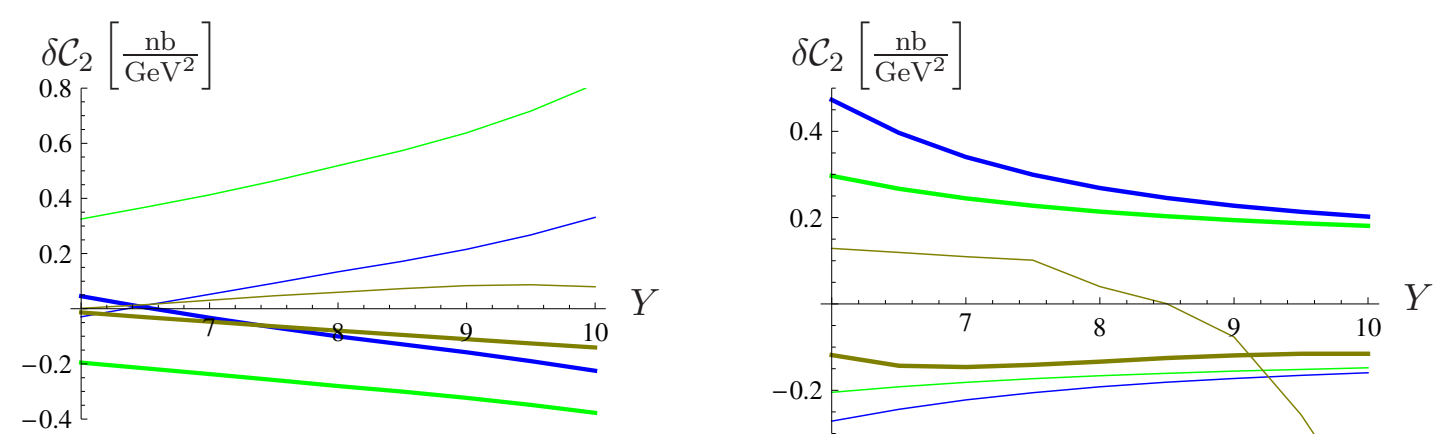

Figure 36. Relative effect of changing $\mu_{R}=\mu_{F}$ by factors 2 and $1 / 2$ respectively (left), and $\sqrt{s_{0}}$ (right) by factors 2 and $1 / 2$ respectively on the coefficient $\mathcal{C}_{2}$ in dependence on $Y$ for $\left|\mathbf{k}_{J, 1}\right|=$ $\left|\mathbf{k}_{J, 2}\right|=50 \mathrm{GeV}$. The tabled values are shown in tables 39 and 40 . 
some kind of Sudakov resummation effects which are not completely under control at the moment [44], even leading to a negative cross-section for $\left|\mathbf{k}_{J, 1}\right|=\left|\mathbf{k}_{J, 2}\right|=35 \mathrm{GeV}$. These prediction are much more stable in the asymmetric configuration. Our own predictions for the cross-section, for $\langle\cos \varphi\rangle,\langle\cos 2 \varphi\rangle$, and $\langle\cos 2 \varphi\rangle /\langle\cos \varphi\rangle$ are given respectively in figures 37, 39, 42 and 45 .

Due to the factorization, the sensitivity of our prediction with respect to $s_{0}, \mu_{R}$ is similar to the two previous symmetrical configurations, as shown in figures 38 for $\mathcal{C}_{0}$, in figures 40,41 for $\mathcal{C}_{1} / \mathcal{C}_{0}$, in figures 43,44 for $\mathcal{C}_{2} / \mathcal{C}_{0}$ and in figures 46,47 for $\mathcal{C}_{2} / \mathcal{C}_{1}$. In figures 48, 49 and figures 50,51, detailed studies for separate coefficients $\mathcal{C}_{1}$ and $\mathcal{C}_{2}$ are displayed.

One sees from figure 37 that our pure NLL prediction, as well as our resummed NLL prediction, are a bit below the NLO-DGLAP prediction, while the LL prediction is much higher than the NLO-DGLAP prediction. The combined LL vertices plus resummed NLL Green's function is rather close to the NLO-DGLAP prediction. One may expect that including higher order corrections in both DGLAP and BFKL approaches would make them converging. We note however that comparing both kinds of treatment should be done with some cautious. Indeed, the NLO-DGLAP involves scales which are smaller than the scale which we consider: we take $\mu_{R}=\sqrt{\left|\mathbf{k}_{J, 1}\right| \cdot\left|\mathbf{k}_{J, 2}\right|}$ which is similar to $\left(\left|\mathbf{k}_{J, 1}\right|+\left|\mathbf{k}_{J, 2}\right|\right) / 2$, while the NLO-DGLAP calculation uses the scale $\left(\left|\mathbf{k}_{J, 1}\right|+\left|\mathbf{k}_{J, 2}\right|\right) / 4$. Changing this scale from $\left(\left|\mathbf{k}_{J, 1}\right|+\left|\mathbf{k}_{J, 2}\right|\right) / 4$ to $\left(\left|\mathbf{k}_{J, 1}\right|+\left|\mathbf{k}_{J, 2}\right|\right) / 8$ leads to a variation of the order of $5 \%$ in the NLO-DGLAP prediction. Our treatment, especially when considering the azimuthal decorrelation, favors higher scales, like $\sqrt{\left|\mathbf{k}_{J, 1}\right| \cdot\left|\mathbf{k}_{J, 2}\right|} \sim\left(\left|\mathbf{k}_{J, 1}\right|+\left|\mathbf{k}_{J, 2}\right|\right) / 2$ or even $2 \sqrt{\left|\mathbf{k}_{J, 1}\right| \cdot\left|\mathbf{k}_{J, 2}\right|} \sim\left|\mathbf{k}_{J, 1}\right|+\left|\mathbf{k}_{J, 2}\right|$.

The azimuthal decorrelation, which is expected to be the best signal, is predicted to be similar in magnitude and shape both from our pure NLL prediction and our resummed NLL prediction and from the NLO-DGLAP approach, as can be seen from figures 39 and 42. Note however that the uncertainties of our predictions are rather high. Anyway, the general trend is clear: the azimuthal decorrelation is much lower than expected from a LL BFKL treatment or from a mixed treatment with LL vertices combined with NLL Green's function. It is also rather flat with $Y$. The only observable which still remain different when comparing pure NLL approaches (the resummed NLL approach makes no difference here since it only affects $\mathcal{C}_{0}$ ) with NLO-DGLAP is the ratio $\langle\cos 2 \varphi\rangle /\langle\cos \varphi\rangle$ for which the NLODGLAP is still significantly higher than the NLL prediction, as can be seen from figure 45. 


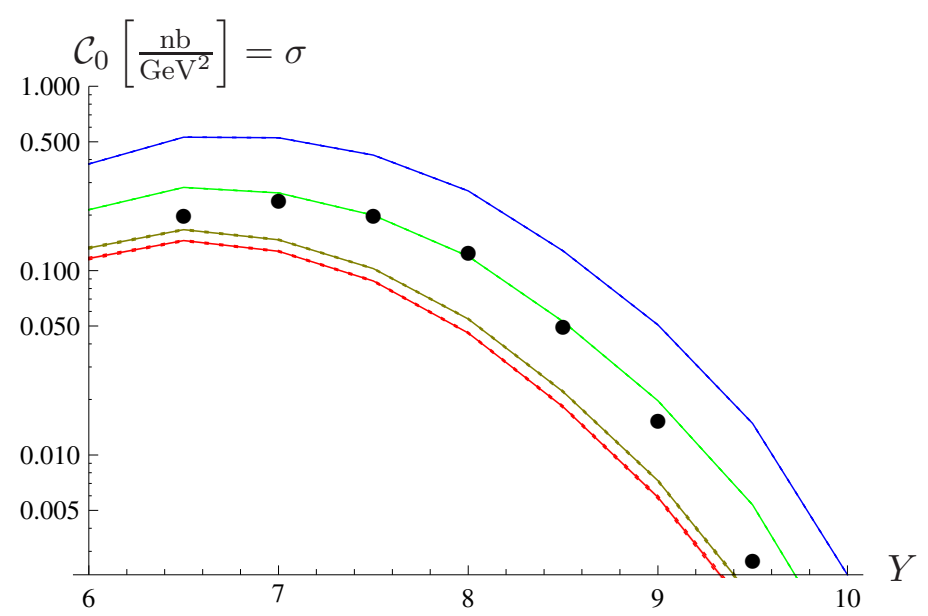

Figure 37. Differential cross section in dependence on $Y$ for $\left|\mathbf{k}_{J, 1}\right|=35 \mathrm{GeV},\left|\mathbf{k}_{J, 2}\right|=50 \mathrm{GeV}$. The errors due to the Monte Carlo integration - though hardly visible - are given as error bands. The tabled values are shown in table 41. As dots are shown the results of ref. [42] obtained with DiJET [43].
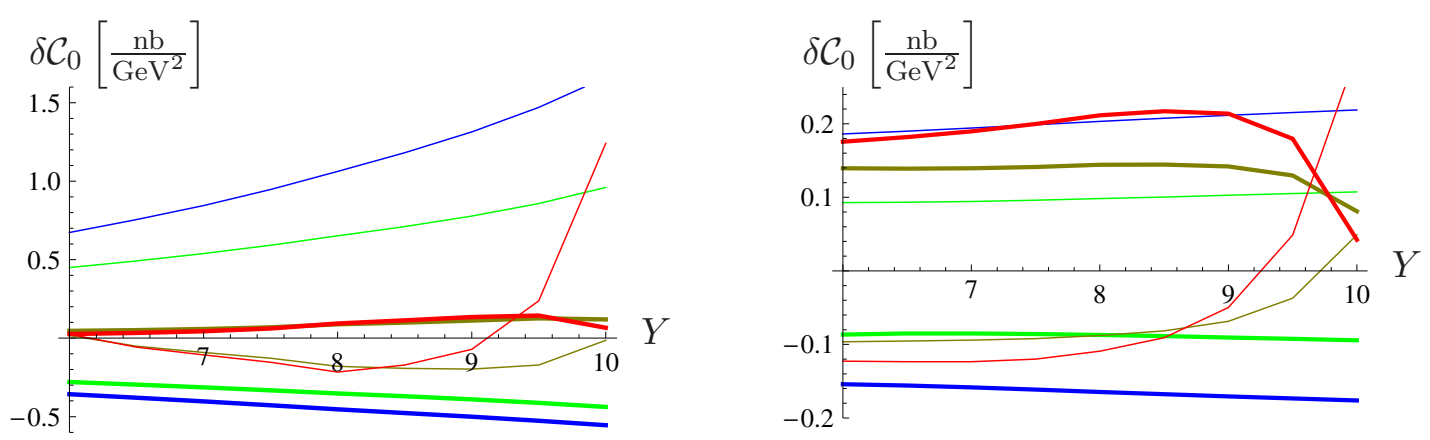

Figure 38. Relative effect of changing $\mu_{R}=\mu_{F}$ by factors 2 and $1 / 2$ respectively (left), and $\sqrt{s_{0}}$ (right) by factors 2 and $1 / 2$ respectively on the differential cross section in dependence on $Y$ for $\left|\mathbf{k}_{J, 1}\right|=35 \mathrm{GeV}, \quad\left|\mathbf{k}_{J, 2}\right|=50 \mathrm{GeV}$. The tabled values are shown in tables 42 and 43 . 


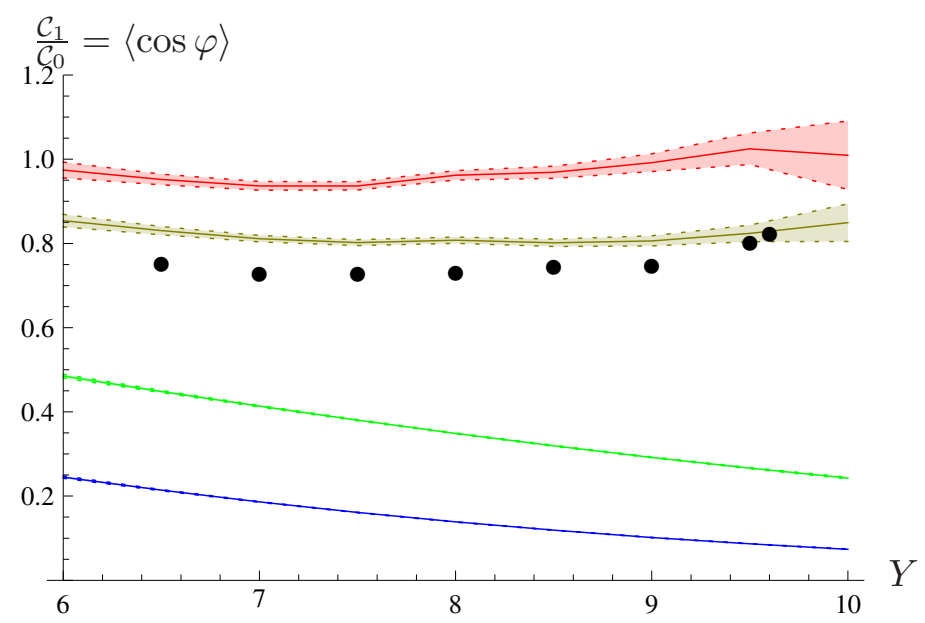

Figure 39. $\langle\cos \varphi\rangle$ in dependence on $Y$ for $\left|\mathbf{k}_{J, 1}\right|=35 \mathrm{GeV},\left|\mathbf{k}_{J, 2}\right|=50 \mathrm{GeV}$. The errors due to the Monte Carlo integration are given as error bands. The tabled values are shown in table 44. As dots are shown the results of ref. [42] obtained with DiJET [43].
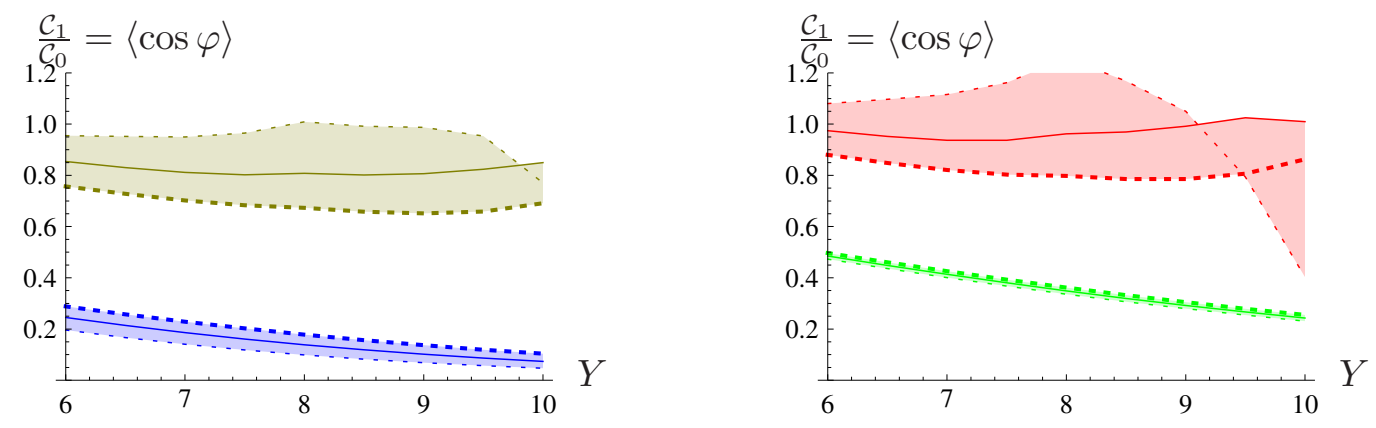

Figure 40. Effect of changing $\mu_{R}=\mu_{F}$ by factors 2 and $1 / 2$ respectively on $\langle\cos \varphi\rangle$ in dependence on $Y$ for $\left|\mathbf{k}_{J, 1}\right|=35 \mathrm{GeV},\left|\mathbf{k}_{J, 2}\right|=50 \mathrm{GeV}$. The tabled values are shown in table 45
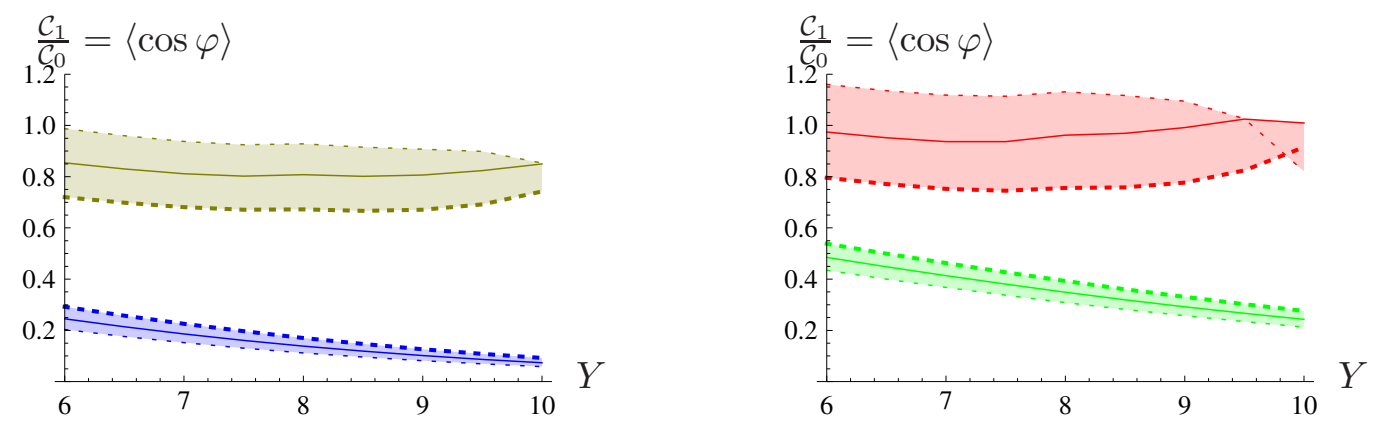

Figure 41. Effect of changing $\sqrt{s_{0}}$ by factors 2 and $1 / 2$ respectively on $\langle\cos \varphi\rangle$ in dependence on $Y$ for $\left|\mathbf{k}_{J, 1}\right|=35 \mathrm{GeV},\left|\mathbf{k}_{J, 2}\right|=50 \mathrm{GeV}$. The tabled values are shown in table 46 . 


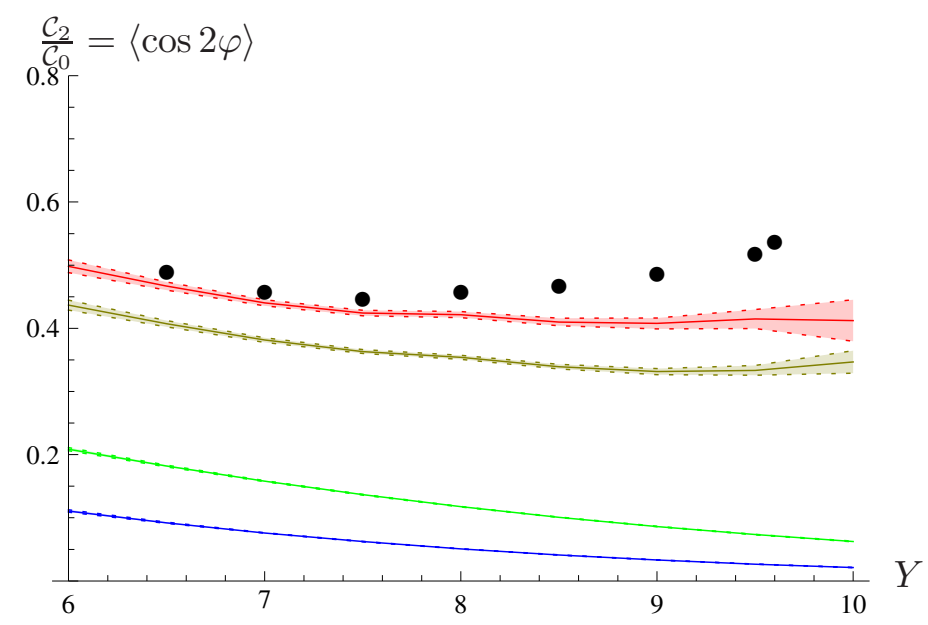

Figure 42. $\langle\cos 2 \varphi\rangle$ in dependence on $Y$ for $\left|\mathbf{k}_{J, 1}\right|=35 \mathrm{GeV},\left|\mathbf{k}_{J, 2}\right|=50 \mathrm{GeV}$. The errors due to the Monte Carlo integration are given as error bands. The tabled values are shown in table 47. As dots are shown the results of ref. [42] obtained with DiJET [43].
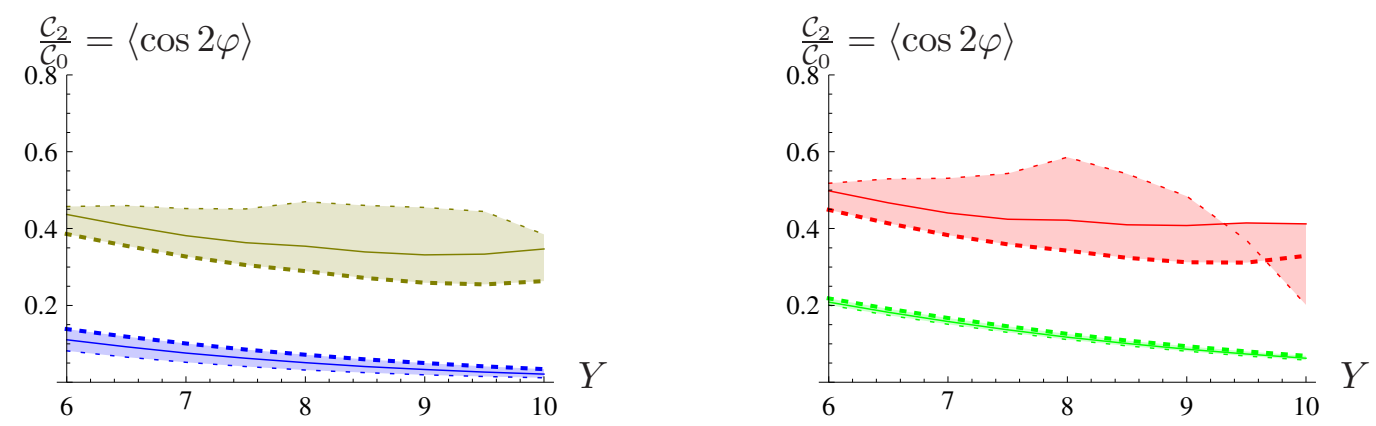

Figure 43. Effect of changing $\mu_{R}=\mu_{F}$ by factors 2 and $1 / 2$ respectively on $\langle\cos 2 \varphi\rangle$ in dependence on $Y$ for $\left|\mathbf{k}_{J, 1}\right|=35 \mathrm{GeV},\left|\mathbf{k}_{J, 2}\right|=50 \mathrm{GeV}$. The tabled values are shown in table 48
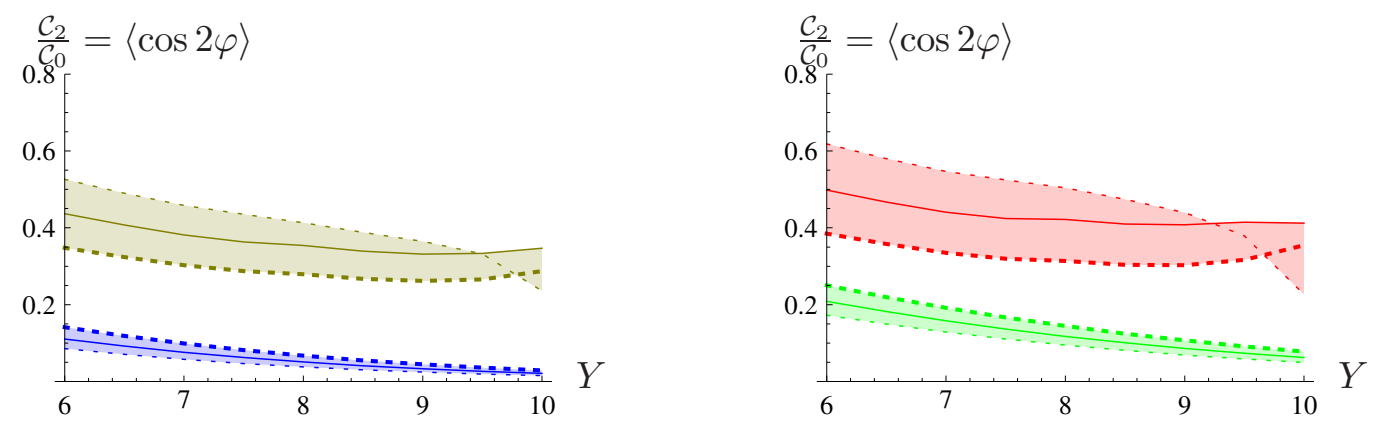

Figure 44. Effect of changing $\sqrt{s_{0}}$ by factors 2 and $1 / 2$ respectively on $\langle\cos 2 \varphi\rangle$ in dependence on $Y$ for $\left|\mathbf{k}_{J, 1}\right|=35 \mathrm{GeV},\left|\mathbf{k}_{J, 2}\right|=50 \mathrm{GeV}$. The tabled values are shown in table 49 . 


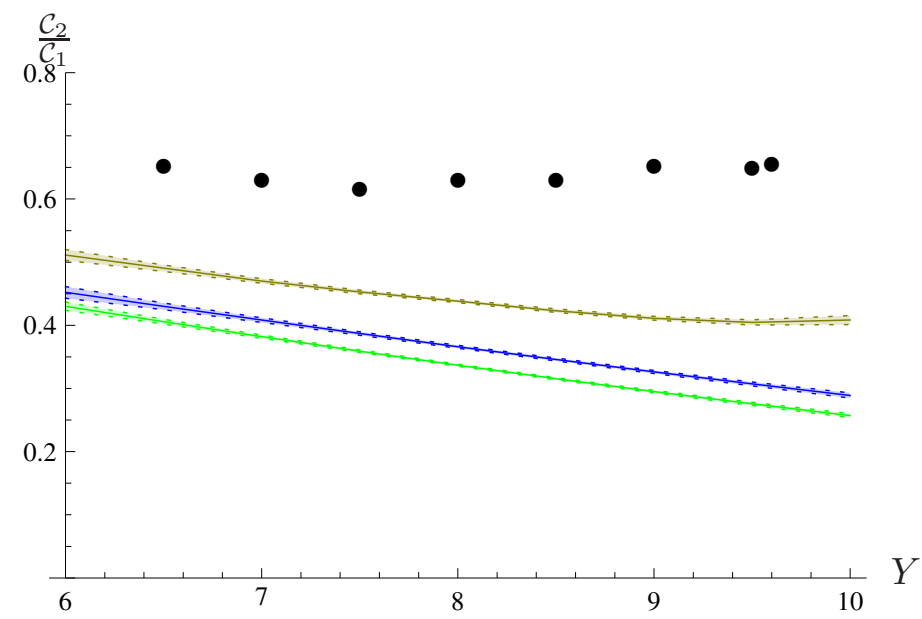

Figure 45. $\langle\cos 2 \varphi\rangle /\langle\cos \varphi\rangle$ in dependence on $Y$ for $\left|\mathbf{k}_{J, 1}\right|=35 \mathrm{GeV},\left|\mathbf{k}_{J, 2}\right|=50 \mathrm{GeV}$. The errors due to the Monte Carlo integration - though hardly visible - are given as error bands. The tabled values are shown in table 50. As dots are shown the results of ref. [42] obtained with DiJET [43].
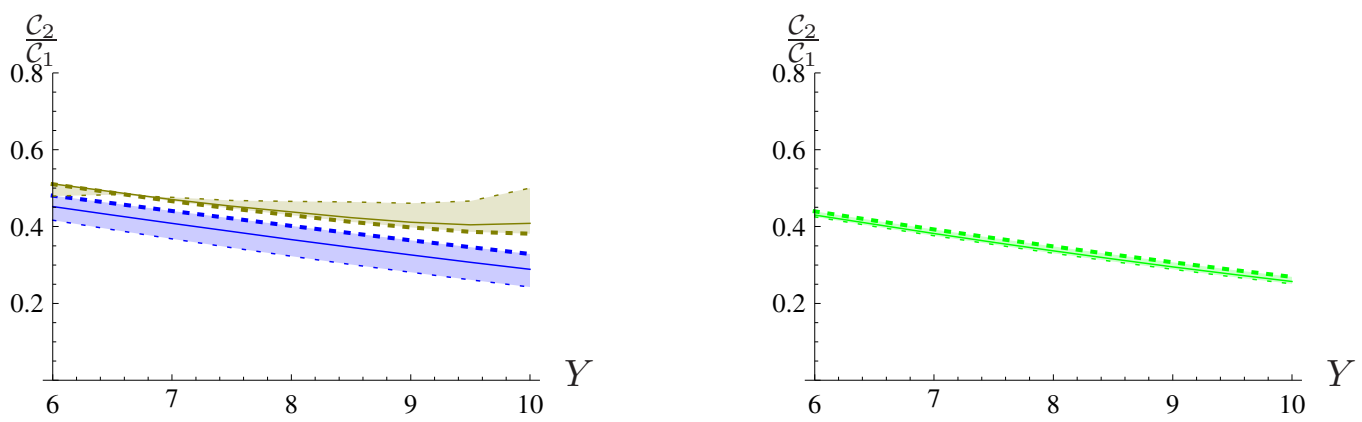

Figure 46. Effect of changing $\mu_{R}=\mu_{F}$ by factors 2 and $1 / 2$ respectively on $\langle\cos 2 \varphi\rangle /\langle\cos \varphi\rangle$ in dependence on $Y$ for $\left|\mathbf{k}_{J, 1}\right|=35 \mathrm{GeV},\left|\mathbf{k}_{J, 2}\right|=50 \mathrm{GeV}$. The tabled values are shown in table 51
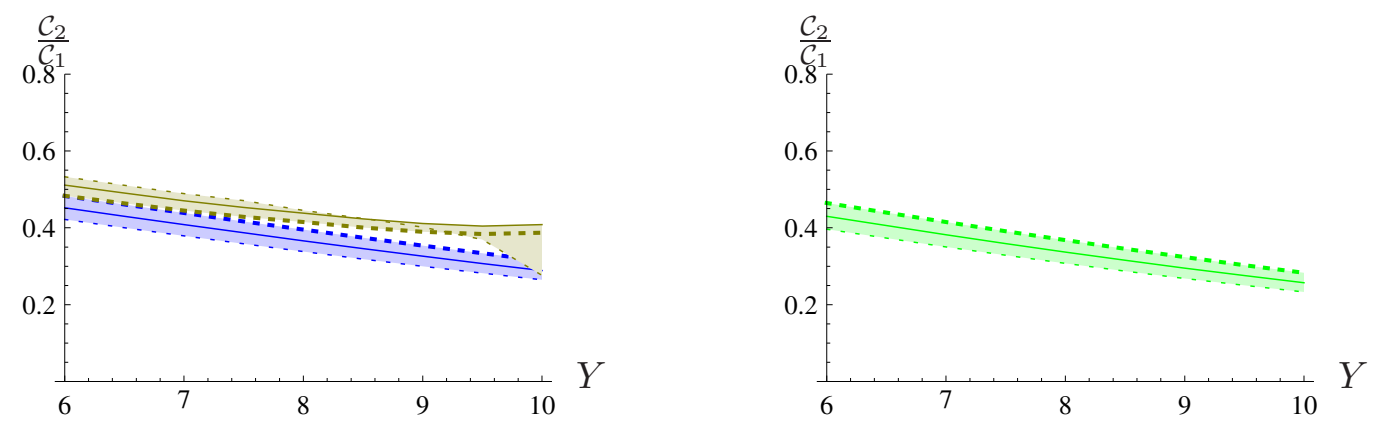

Figure 47. Effect of changing $\sqrt{s_{0}}$ by factors 2 and $1 / 2$ respectively on $\langle\cos 2 \varphi\rangle /\langle\cos \varphi\rangle$ in dependence on $Y$ for $\left|\mathbf{k}_{J, 1}\right|=35 \mathrm{GeV},\left|\mathbf{k}_{J, 2}\right|=50 \mathrm{GeV}$. The tabled values are shown in table 52 . 


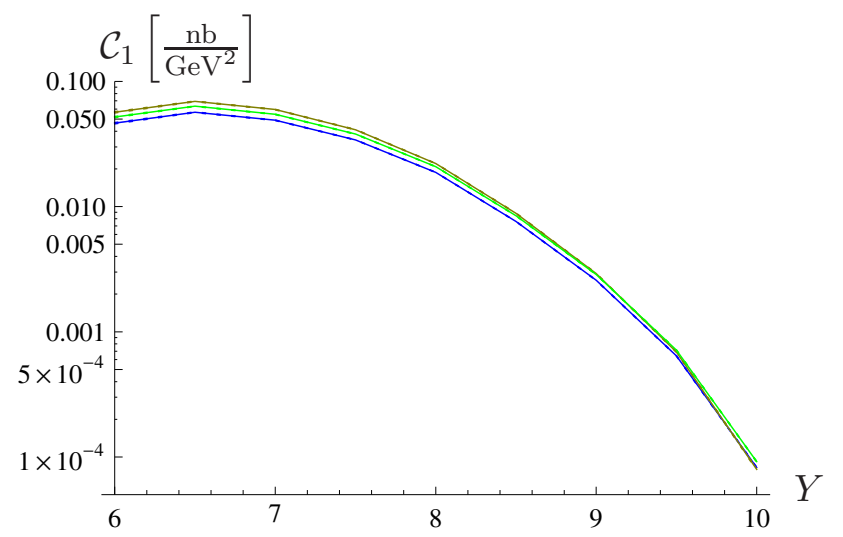

Figure 48. Coefficient $\mathcal{C}_{1}$ in dependence on $Y$ for $\left|\mathbf{k}_{J, 1}\right|=35 \mathrm{GeV},\left|\mathbf{k}_{J, 2}\right|=50 \mathrm{GeV}$. The errors due to the Monte Carlo integration - though hardly visible - are given as error bands. The tabled values are shown in table 53 .
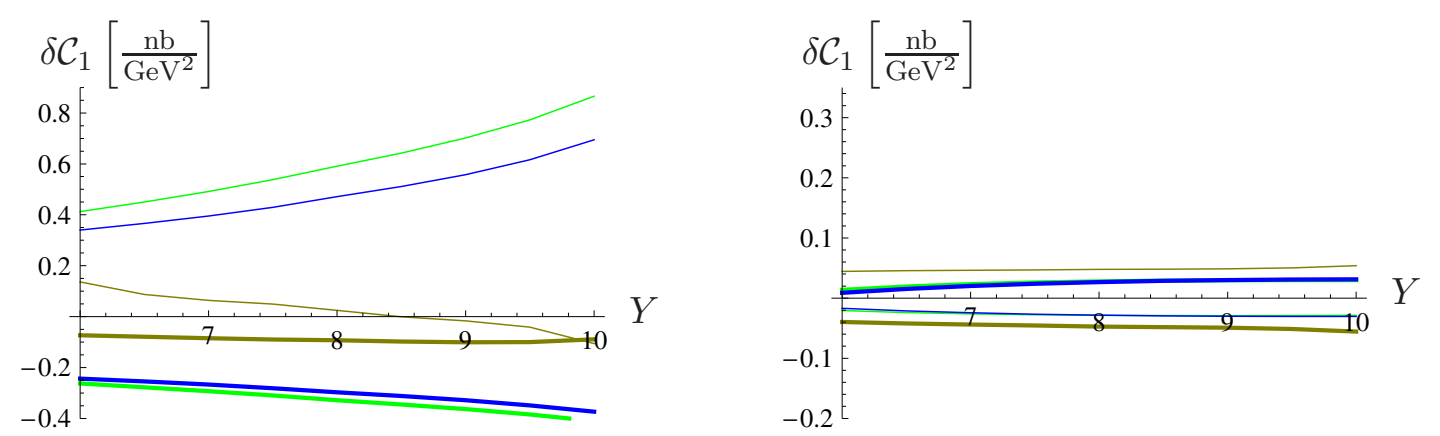

Figure 49. Relative effect of changing $\mu_{R}=\mu_{F}$ by factors 2 and $1 / 2$ respectively (left), and $\sqrt{s_{0}}$ (right) by factors 2 and $1 / 2$ respectively on the coefficient $\mathcal{C}_{1}$ in dependence on $Y$ for $\left|\mathbf{k}_{J, 1}\right|=$ $35 \mathrm{GeV},\left|\mathbf{k}_{J, 2}\right|=50 \mathrm{GeV}$. The tabled values are shown in tables 54 and 55 .

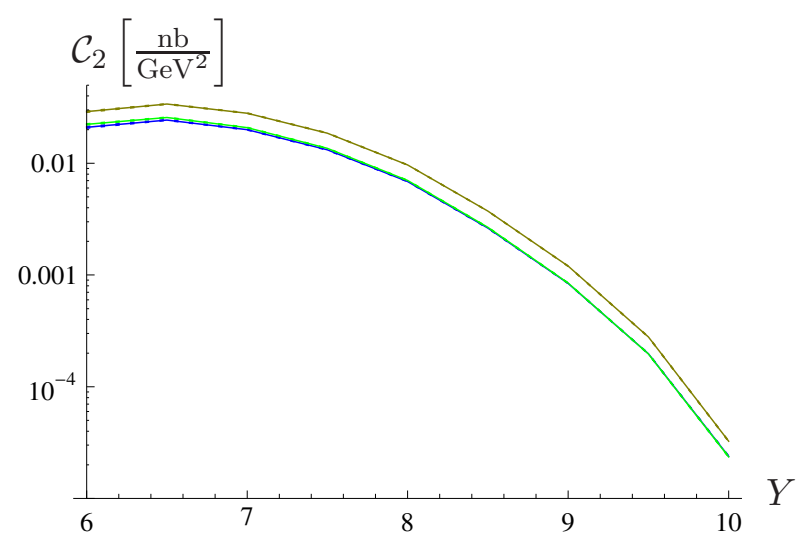

Figure 50. Coefficient $\mathcal{C}_{2}$ in dependence on $Y$ for $\left|\mathbf{k}_{J, 1}\right|=35 \mathrm{GeV},\left|\mathbf{k}_{J, 2}\right|=50 \mathrm{GeV}$. The errors due to the Monte Carlo integration - though hardly visible - are given as error bands. The tabled values are shown in table 56 . 

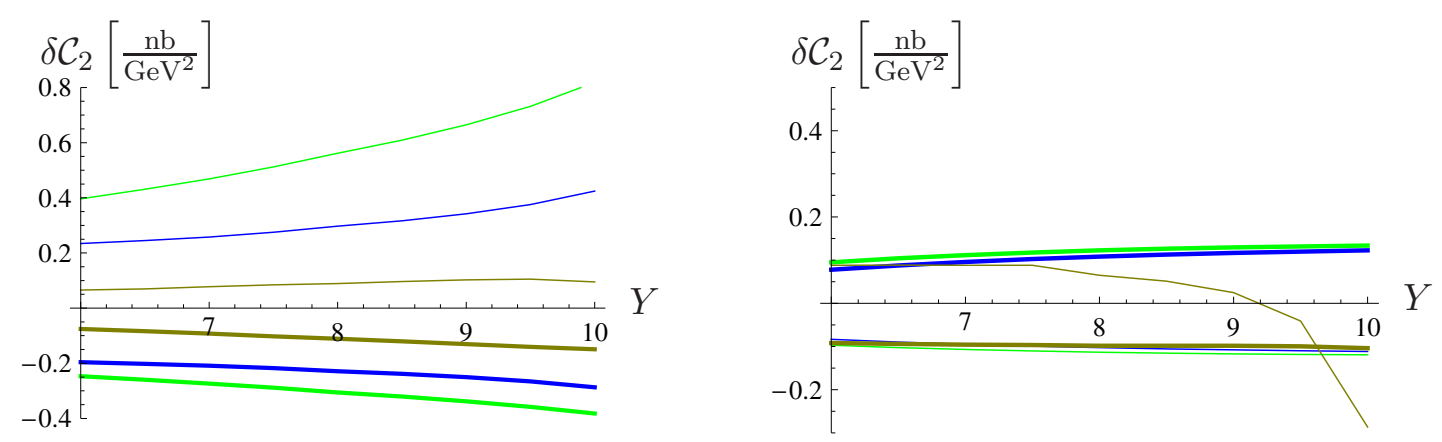

Figure 51. Relative effect of changing $\mu_{R}=\mu_{F}$ by factors 2 and $1 / 2$ respectively (left), and $\sqrt{s_{0}}$ (right) by factors 2 and $1 / 2$ respectively on the coefficient $\mathcal{C}_{2}$ in dependence on $Y$ for $\left|\mathbf{k}_{J, 1}\right|=$ $35 \mathrm{GeV}, \quad\left|\mathbf{k}_{J, 2}\right|=50 \mathrm{GeV}$. The tabled values are shown in tables 57 and 58 .

\section{Conclusions}

We have implemented at full NLL order the Mueller Navelet jets cross-section as well as their relative azimuthal angle dependency. In contrast to the general belief, the effect of NLL corrections to the vertex function is very important, of the same order as the one obtained when passing from LL to NLL Green's function. The importance of NLL corrections to the impact factor observed in the present paper is analogous to recent results obtained at NLL in diffractive double $\rho$-electroproduction $[23,40]$. Interestingly, the full NLL calculations for $\langle\cos \varphi\rangle$ and $\langle\cos 2 \varphi\rangle$ are quite close to a calculation [42] using DiJeT [43] which is based on DGLAP dynamics and to a dedicated study [15] using PYTHIA [38] and HERWIG [39]. The uncertainty due to changes in $\mu_{R}$ (and $s_{0}$ ) is drastically reduced for all $\mathcal{C}_{n}$ when one takes into account the NLL Mueller Navelet vertices. The uncertainty due to PDFs are also moderate. As a consequence, our results for the cross-section are very stable.

However, for azimuthal decorrelation the dependence on $\mu_{R}$ (and $s_{0}$ ) is still sizeable. In the case of the NLL Green's function with collinear improvement one observes that $\langle\cos \varphi\rangle$ can exceed 1 for certain choices of the parameters, in particular for low values of $\mu_{R}=\mu_{F}$, taken to be smaller than the "natural" value $\sqrt{\left|k_{J, 1}\right| \cdot\left|k_{J, 2}\right|}$. One might also think of a collinear improvement of the vertices [33] but the Mueller Navelet vertex for fixed $\left|\mathbf{k}_{J}\right|$ does not have poles in $\gamma$ nor $1-\gamma$, so there is no room for such a treatment. The resummation of soft initial radiation might be of relevance for the azimuthal correlation as well. This is left for further investigations, and in this work we rather consider the full NLL calculation without additional collinear resummation to be our solid prediction, while the 'collinear improvement' as it stands is not appropriate to study azimuthal dependences.

At present, there is little experience with the effect of NLL impact factors. To the best of our knowledge, up to now, the only full NLL BFKL calculation existing in the literature is the vector meson production in virtual photon collisions [23, 40, 41], which is very sensitive to NLL corrections to the impact factor and for which very large values for $s_{0}$ and $\mu_{R}$ are preferred. In [41] it has been shown that a collinear improved treatment combined with the application of the principle of minimal sensitivity $[45,46]$ reduces this large values to more 
"natural" values. Still, $\mu_{R}$ larger than the "natural" values are favored [41]. In the present case, with the scales $\mu_{R}$ and $s_{0}$ set by the jet scale, we get azimuthal correlations which are rather similar to DGLAP dynamics predictions (although, as we already mentioned, the DGLAP prediction are based on smaller scales). To conclude, contrarily to the expectation, it thus seems that the azimuthal decorrelation is almost not enhanced by an increasing rapidity. This suggests that the study of Mueller Navelet jets is probably not the best place to exhibit differences between BFKL and DGLAP dynamics.

\section{Acknowledgments}

We acknowledge discussions with Jochen Bartels, Salim Cerci, David d'Enterria, Bernard Pire, Agustín Sabio Vera, Kirsten Sachs, Gian Paolo Vacca. We especially thank Michel Fontannaz for many explanations of the DGLAP-based approach and for providing us his own predictions. This work is supported in part by the Polish Grant N202 249235, the French-Polish scientific agreement Polonium, by the grant ANR-06-JCJC-0084 and by the ECO-NET program, contract $12584 \mathrm{QK}$, and by a PRIN grant (MIUR, Italy).

\section{A Details on the numerical implementation}

\section{A.1 Programs used}

We implemented all numerical calculations in Mathematica. To this purpose we used the according interfaces for the MSTW 2008 PDFs [16] and for version 1.5 of CuBA [37] which we used for numerical integration.

\section{A.2 Choice of parameters}

CuBA provides different integration routines which we also used to cross-check the results of the Monte Carlo integration. However, for the final results we used the Vegas routine of CUBA with an aimed precision of $10^{-2}$ and a maximal number of 500000 points per integration. To use a Monte Carlo integrator, all integration intervals have to be mapped on finite intervals. For the transverse momentum integrations we used the mapping $|\mathbf{k}|=\left|\mathbf{k}_{J}\right| \tan (\xi \pi / 2)$.

The cancellations which analytically have been shown in refs. $[13,14]$ numerically can corrupt the integration due to the limited precision of a computer. In all these cases, were the integration interval have been mapped to the compact interval $[0,1]$, we used a cut off of $10^{-5}$ where the cut off dependence becomes negligible.

\section{A.2.1 The $\nu$-grid}

Due to the complicate matrix element, the PDF evaluation, and the implementation in Mathematica instead of a dedicated stand-alone code the Monte Carlo integration is very time consuming. Therefor, the choice of the $\nu$-values at which the coefficients $C_{n, \nu}(2.19)$ are evaluated is crucial.

We are guided by the shape of the BFKL Green's function which is peaked around $\nu=0$ and then monotonically falls. The smaller $Y$ the slower the decrease. Even though 
the minimal $Y$ in this study is 6 , we want our coefficients to be prepared also for smaller $Y$ 's. We choose a maximal $\nu_{\max }$ such that an integration up to $\nu_{\max }$ of just the NLL BFKL Green's function at $Y=4$ for $n=0$ reproduces $96 \%$ of the integration over the full $\nu$-range. For the case of $Y=6$ it reproduces $99.97 \%$ of the full integral.

The coefficients $C_{n, \nu}$ are oscillating like $\exp \left(i \nu \ln \mathbf{k}_{J, i}^{2}\right)$ but more important is the product of the two which has an oscillating part with a frequency $\nu_{\text {oscillation }}=\pi / \ln \frac{\left|\mathbf{k}_{J, 1}\right|}{\left|\mathbf{k}_{J, 2}\right|}$. This frequency is zero for $\left|\mathbf{k}_{J, 1}\right|=\left|\mathbf{k}_{J, 2}\right|$ but for the example of $\left|\mathbf{k}_{J, 1}\right|=35 \mathrm{GeV}$ and $\left|\mathbf{k}_{J, 2}\right|=50 \mathrm{GeV}$ we chose a step width for $\nu$ of $\nu_{\text {oscillation }} / 4$.

For large $Y$ it is really the small region close to $\nu=0$ which matters. Therefore we sample this region in more detail according to the shape of the NLL BFKL Green's function for $n=0$ and $Y=7$. The final $\nu$-grid reads

$$
\begin{array}{r}
\{0,0.0334439,0.0671152,0.101257,0.136128,0.172017,0.209264,0.248284, \\
0.289594,0.333866,0.382007,0.435281,0.495535,0.565607,0.65013,0.75725, \\
\left.0.902736,1.12137,1.50735,2.44882, \frac{2 \pi}{4 \ln \frac{10}{7}}, \frac{3 \pi}{4 \ln \frac{10}{7}}, \ldots, \frac{49 \pi}{4 \ln \frac{10}{7}}\right\}
\end{array}
$$

For the final integration over $\nu$ the product $C_{n, \nu}\left(\left|\mathbf{k}_{J, 1}\right|, x_{J, 1}\right) C_{n, \nu}^{*}\left(\left|\mathbf{k}_{J, 2}\right|, x_{J, 2}\right)$ is interpolated by cubic splines.

\section{A.3 Grouping the integrand}

In this section we describe how the NLL contribution to the coefficients $C_{n, \nu}$, as defined in eq. (2.19), is arranged.

The jet defining function $\mathcal{S}_{J}^{(3)}$ given in eq. (3.6) consists of three parts which we label $\mathcal{S}_{J, a}^{(3)}, \mathcal{S}_{J, b}^{(3)}$ and $\mathcal{S}_{J, c}^{(3)}$. With this separation $V_{\mathrm{q}}^{(1)}$, given in eq. (3.5), consists of 17 elementary blocks which we denote by $V_{\mathrm{q}}[i]$ (suppressing for the time being all further arguments and indices), where $i=1, \ldots, 17$. In the same spirit we decompose $V_{\mathrm{g}}^{(1)}$ given in eq. (3.4) in its 25 elementary blocks $V_{\mathrm{g}}[i]$.

It is useful to replace the integration variable $\mathbf{k}$ by $\mathbf{k} \rightarrow \mathbf{k}_{J}-\mathbf{k}$ in the integrands $V_{\mathrm{q}}[5]$ and $V_{\mathrm{q}}[7]\left(V_{\mathrm{g}}[23]\right.$ and $\left.V_{\mathrm{g}}[25]\right)$. Moreover we split up $V_{\mathrm{q}}[3]\left(V_{\mathrm{g}}[21]\right)$ and create $V_{\mathrm{q}}[18]\left(V_{\mathrm{g}}[26]\right)$ where in the new elementary blocks the integrand $\mathbf{k}^{\prime}$ is replaced by $\mathbf{k}_{J}-\mathbf{k}^{\prime}$. Then we make the following replacements

$$
\begin{gathered}
V_{\mathrm{q}}[3] \rightarrow V_{\mathrm{q}}[3] \Theta\left(z-\frac{1}{2}\right)+V_{\mathrm{q}}[18] \Theta\left(\frac{1}{2}-z\right) \\
V_{\mathrm{g}}[21] \rightarrow V_{\mathrm{g}}[21] \Theta\left(z-\frac{1}{2}\right)+V_{\mathrm{g}}[26] \Theta\left(\frac{1}{2}-z\right) .
\end{gathered}
$$

The elementary blocks are now grouped to 14 minimal basic blocks $B[i]$ which also contain the integrations from eq. (2.19)

$$
B[1]=\iiint \int V_{\mathrm{q}}[1]
$$




$$
\begin{aligned}
& B[2]=\iiint \int V_{\mathrm{q}}[2] \\
& B[3]=\iiint \int\left(V_{\mathrm{q}}[3] \Theta\left(z-\frac{1}{2}\right)+V_{\mathrm{q}}[4]+V_{\mathrm{q}}[6]\right) \\
& B[4]=\iiint \int\left(V_{\mathrm{q}}[18] \Theta\left(\frac{1}{2}-z\right)+V_{\mathrm{q}}[5]+V_{\mathrm{q}}[7]\right) \\
& B[5]=\iiint \int\left(V_{\mathrm{q}}[11]+V_{\mathrm{q}}[12]+V_{\mathrm{q}}[13]+V_{\mathrm{q}}[15]+\frac{1}{2}\left(V_{\mathrm{q}}[16]+V_{\mathrm{q}}[17]\right)\right) \\
& B[6]=\iiint \int\left(V_{\mathrm{q}}[8]+V_{\mathrm{q}}[9]+V_{\mathrm{q}}[10]+V_{\mathrm{q}}[14]+\frac{1}{2}\left(V_{\mathrm{q}}[16]+V_{\mathrm{q}}[17]\right)\right) \\
& B[7]=\iiint \int V_{\mathrm{g}}[1] \\
& B[8]=\iiint \int V_{\mathrm{g}}[2] \\
& B[9]=\iiint \int \sum_{i=3}^{6} V_{\mathrm{g}}[i] \\
& B[10]=\iiint \int \sum_{i=7}^{10} V_{\mathrm{g}}[i] \\
& B[11]=\iiint \int\left(V_{\mathrm{g}}[11]+V_{\mathrm{g}}[12]+V_{\mathrm{g}}[13]+V_{\mathrm{g}}[17]+\frac{1}{2}\left(V_{\mathrm{g}}[19]+V_{\mathrm{g}}[20]\right)\right) \\
& B[12]=\iiint \int\left(V_{\mathrm{g}}[14]+V_{\mathrm{g}}[15]+V_{\mathrm{g}}[16]+V_{\mathrm{g}}[18]+\frac{1}{2}\left(V_{\mathrm{g}}[19]+V_{\mathrm{g}}[20]\right)\right) \\
& B[13]=\iiint \int\left(V_{\mathrm{g}}[21] \Theta\left(z-\frac{1}{2}\right)+V_{\mathrm{g}}[22]+V_{\mathrm{g}}[24]\right) \\
& B[14]=\iiint \int\left(V_{\mathrm{g}}[26] \Theta\left(\frac{1}{2}-z\right)+V_{\mathrm{g}}[23]+V_{\mathrm{g}}[25]\right),
\end{aligned}
$$

where we made use of the short hand notation $\iiint \int \equiv \int \mathrm{d} \phi_{J} \mathrm{~d}^{2} \mathbf{k} \mathrm{d} x f(x) E_{n, \nu}(\mathbf{k}) \cos \left(m \phi_{J}\right)$.

We would like to point out that the inclusion of $V_{\mathrm{q}}[18]\left(V_{\mathrm{g}}[26]\right)$ in $B[4](B[14])$ is essential. Even though it is correctly stated after eq. (88) in ref. [13] (and repeated in ref. [14] after eq. (53)), that in the composite jet configuration the domain of integration shrinks like $z^{2}$ for $z \rightarrow 0$, the conclusion that this prevents a divergence is wrong. In fact, in the limit $z \rightarrow 0$ the integrand scales like $z^{-3}$ and only the sum of $V_{\mathrm{q}}[18]\left(V_{\mathrm{g}}[26]\right)$ and $V_{\mathrm{q}}[5]\left(V_{\mathrm{g}}[23]\right)$ cancels properly against $V_{\mathrm{q}}[7]\left(V_{\mathrm{g}}[25]\right)$ in the dangerous region.

Note that in case of fix $x_{J}$ for $B[1]$ and $B[7]$ no numerical integration is needed, while for $B[2]$ and $B[8]$ only one integration over $z$ has to be done. All four are proportional to the LL Mueller Navelet vertex regarding the transverse momentum dependences. The Dirac- $\delta$ in transverse momenta we always use for the $\mathbf{k}$ integration. Only for contributions with $\delta^{(2)}\left(\mathbf{k}^{\prime}-\mathbf{k}_{J}\right)$ it is used for the $\mathbf{k}^{\prime}$ integration. The $x$-integration is trivially performed by evaluating the according Dirac $\delta$-distribution. 


\begin{tabular}{|l|l|l|l|l|}
\hline & LL & \multicolumn{3}{|c|}{ NLL } \\
$Y$ & & MC0 & MC+ & MC- \\
\hline 6 & 1.52 & 0.606 & 0.006 & -0.006 \\
6.5 & 2.06 & 0.752 & 0.005 & -0.005 \\
7 & 2.04 & 0.670 & 0.004 & -0.004 \\
7.5 & 1.70 & 0.490 & 0.003 & -0.003 \\
8 & 1.18 & 0.289 & 0.002 & -0.002 \\
8.5 & 0.602 & 0.126 & 0.0009 & -0.0009 \\
9 & 0.268 & 0.0474 & 0.0004 & -0.0004 \\
9.5 & 0.0949 & 0.0138 & 0.0002 & -0.0002 \\
10 & 0.0207 & 0.00238 & 0.00006 & -0.00006 \\
\hline & LL+ & \multicolumn{3}{|c|}{ NLL+ } \\
$Y$ & & MC0 & MC+ & MC- \\
\hline 6 & 0.849 & 0.549 & 0.006 & -0.006 \\
6.5 & 1.10 & 0.675 & 0.006 & -0.006 \\
7 & 1.03 & 0.595 & 0.004 & -0.004 \\
7.5 & 0.804 & 0.430 & 0.003 & -0.003 \\
8 & 0.523 & 0.249 & 0.002 & -0.002 \\
8.5 & 0.248 & 0.107 & 0.001 & -0.001 \\
9 & 0.102 & 0.0396 & 0.0005 & -0.0005 \\
9.5 & 0.0337 & 0.0114 & 0.0002 & -0.0002 \\
10 & 0.00681 & 0.00198 & 0.00008 & -0.00008 \\
\hline
\end{tabular}

Table 1. Tabled values for figure 3,5 .

\section{B Tabled values of diagrams}

To allow for later accurate comparisons, we give the values for all plots in this work. We mark the pure LL calculation by 'LL', and the pure NLL one by 'NLL'. The combination of LL vertices with the NLL collinear improved Green's function is denoted as 'LL+', and the combination of NLL vertices with the NLL collinear improved Green's function as 'NLL+'. Whenever in a figure the effect of the variation of one parameter is presented, in the according table the first column shows the central value, while the second and third show the change of this central value due to the varied parameter. For brevity we suppress the energy unit $\mathrm{GeV}$ in the headings of the tables. 


\begin{tabular}{|l|l|l|l|l|l|l|}
\hline & \multicolumn{3}{|c|}{ LL } & \multicolumn{3}{c|}{ NLL } \\
$Y$ & $\mu=35$ & $\mu=70$ & $\mu=17.5$ & $\mu=35$ & $\mu=70$ & $\mu=17.5$ \\
\hline 6 & 1.52 & -0.4 & 0.8 & 0.606 & 0.03 & -0.04 \\
6.5 & 2.06 & -0.6 & 1. & 0.752 & 0.03 & -0.06 \\
7 & 2.04 & -0.7 & 2. & 0.670 & 0.03 & -0.06 \\
7.5 & 1.70 & -0.7 & 2. & 0.490 & 0.02 & -0.05 \\
8 & 1.18 & -0.5 & 1. & 0.289 & 0.02 & -0.03 \\
8.5 & 0.602 & -0.3 & 0.7 & 0.126 & 0.007 & -0.02 \\
9 & 0.268 & -0.1 & 0.4 & 0.0474 & 0.003 & -0.006 \\
9.5 & 0.0949 & -0.05 & 0.1 & 0.0138 & 0.001 & -0.002 \\
10 & 0.0207 & -0.01 & 0.03 & 0.00238 & 0.0002 & -0.0002 \\
\hline & \multicolumn{5}{|c|}{ LL+ +} & \multicolumn{3}{|c|}{ NLL+ } \\
$Y$ & $\mu=35$ & $\mu=70$ & $\mu=17.5$ & $\mu=35$ & $\mu=70$ & $\mu=17.5$ \\
\hline 6 & 0.849 & -0.2 & 0.3 & 0.549 & 0.02 & -0.04 \\
6.5 & 1.10 & -0.3 & 0.5 & 0.675 & 0.02 & -0.05 \\
7 & 1.03 & -0.3 & 0.5 & 0.595 & 0.02 & -0.05 \\
7.5 & 0.805 & -0.3 & 0.5 & 0.430 & 0.02 & -0.05 \\
8 & 0.523 & -0.2 & 0.3 & 0.249 & 0.01 & -0.03 \\
8.5 & 0.248 & -0.09 & 0.2 & 0.107 & 0.007 & -0.01 \\
9 & 0.102 & -0.04 & 0.08 & 0.0396 & 0.003 & -0.003 \\
9.5 & 0.0337 & -0.01 & 0.03 & 0.0114 & 0.0009 & 0.0008 \\
10 & 0.00681 & -0.003 & 0.006 & 0.00197 & 0.0001 & 0.0009 \\
\hline
\end{tabular}

Table 2. Tabled values for left figure of figure 4 .

\begin{tabular}{|c|c|c|c|c|c|c|}
\hline & \multicolumn{3}{|c|}{$\mathrm{LL}$} & \multicolumn{3}{|c|}{ NLL } \\
\hline$Y$ & $\sqrt{s_{0}}=35$ & $\sqrt{s_{0}}=70$ & $\sqrt{s_{0}}=17.5$ & $\sqrt{s_{0}}=35$ & $\sqrt{s_{0}}=70$ & $\sqrt{s_{0}}=17.5$ \\
\hline 6 & 1.52 & -0.03 & 0.1 & 0.606 & 0.03 & -0.008 \\
\hline 6.5 & 2.06 & -0.1 & 0.3 & 0.752 & 0.03 & -0.02 \\
\hline 7 & 2.04 & -0.2 & 0.3 & 0.670 & 0.02 & -0.02 \\
\hline 7.5 & 1.70 & -0.2 & 0.3 & 0.490 & 0.02 & -0.02 \\
\hline 8 & 1.18 & -0.2 & 0.2 & 0.289 & 0.01 & -0.01 \\
\hline 8.5 & 0.602 & -0.09 & 0.1 & 0.126 & 0.005 & -0.006 \\
\hline 9 & 0.268 & -0.04 & 0.06 & 0.0474 & 0.002 & -0.002 \\
\hline 9.5 & 0.0949 & -0.02 & 0.02 & 0.0138 & 0.0005 & -0.0005 \\
\hline 10 & 0.0207 & -0.004 & 0.005 & 0.00238 & 0.00007 & -0.00002 \\
\hline & \multicolumn{3}{|c|}{$\mathrm{LL}+$} & \multicolumn{3}{|c|}{ NLL+ } \\
\hline$Y$ & $\sqrt{s_{0}}=35$ & $\sqrt{s_{0}}=70$ & $\sqrt{s_{0}}=17.5$ & $\sqrt{s_{0}}=35$ & $\sqrt{s_{0}}=70$ & $\sqrt{s_{0}}=17.5$ \\
\hline 6 & 0.849 & 0.009 & 0.02 & 0.549 & 0.03 & -0.01 \\
\hline 6.5 & 1.10 & -0.02 & 0.05 & 0.675 & 0.03 & -0.03 \\
\hline 7 & 1.03 & -0.03 & 0.06 & 0.595 & 0.03 & -0.03 \\
\hline 7.5 & 0.805 & -0.04 & 0.05 & 0.430 & 0.03 & -0.03 \\
\hline 8 & 0.523 & -0.03 & 0.04 & 0.249 & 0.02 & -0.02 \\
\hline 8.5 & 0.248 & -0.02 & 0.02 & 0.107 & 0.007 & -0.006 \\
\hline 9 & 0.102 & -0.008 & 0.009 & 0.0396 & 0.003 & -0.002 \\
\hline 9.5 & 0.0337 & -0.003 & 0.003 & 0.0114 & 0.0007 & 0.000003 \\
\hline 10 & 0.00681 & -0.0006 & 0.0007 & 0.00197 & 0.00008 & 0.0002 \\
\hline
\end{tabular}

Table 3. Tabled values for right figure of figure 4 . 


\begin{tabular}{|l|l|l|l|l|l|l|}
\hline & \multicolumn{3}{|c|}{ LL } & \multicolumn{3}{|c|}{ NLL } \\
$Y$ & PDF0 & PDF+ & PDF- & PDF0 & PDF + & PDF- \\
\hline 6 & 1.52 & 0.08 & -0.1 & 0.606 & 0.03 & -0.04 \\
6.5 & 2.06 & 0.1 & -0.1 & 0.752 & 0.05 & -0.05 \\
7 & 2.04 & 0.2 & -0.2 & 0.670 & 0.06 & -0.06 \\
7.5 & 1.70 & 0.2 & -0.2 & 0.490 & 0.05 & -0.05 \\
8 & 1.18 & 0.1 & -0.1 & 0.289 & 0.04 & -0.03 \\
8.5 & 0.602 & 0.09 & -0.07 & 0.126 & 0.02 & -0.02 \\
9 & 0.268 & 0.05 & -0.04 & 0.0474 & 0.008 & -0.007 \\
9.5 & 0.0949 & 0.02 & -0.02 & 0.0138 & 0.003 & -0.002 \\
10 & 0.0207 & 0.005 & -0.004 & 0.00238 & 0.0006 & -0.0004 \\
\hline & \multicolumn{1}{|l|}{ LL+ +} & & NLL+ \\
$Y$ & PDF0 & PDF+ & PDF- & PDF0 & PDF+ & PDF- \\
\hline 6 & 0.849 & 0.05 & -0.06 & 0.549 & 0.03 & -0.04 \\
6.5 & 1.10 & 0.07 & -0.08 & 0.675 & 0.05 & -0.05 \\
7 & 1.03 & 0.08 & -0.08 & 0.595 & 0.05 & -0.05 \\
7.5 & 0.805 & 0.08 & -0.08 & 0.430 & 0.05 & -0.04 \\
8 & 0.523 & 0.06 & -0.06 & 0.249 & 0.03 & -0.03 \\
8.5 & 0.248 & 0.04 & -0.03 & 0.107 & 0.02 & -0.01 \\
9 & 0.102 & 0.02 & -0.01 & 0.0396 & 0.007 & -0.006 \\
9.5 & 0.0337 & 0.007 & -0.005 & 0.0114 & 0.002 & -0.002 \\
10 & 0.00681 & 0.002 & -0.001 & 0.00197 & 0.0005 & -0.0004 \\
\hline
\end{tabular}

Table 4. Tabled values for left figure of figure 5 .

\begin{tabular}{|l|c|c|c|c|l|l|}
\hline & \multicolumn{3}{|c|}{ LL } & \multicolumn{3}{c|}{ NLL } \\
$Y$ & MC0 & MC+ & MC- & MC0 & MC+ & MC- \\
\hline 6 & 0.377 & 0.003 & -0.003 & 0.851 & 0.01 & -0.01 \\
6.5 & 0.308 & 0.002 & -0.002 & 0.824 & 0.007 & -0.007 \\
7 & 0.252 & 0.0009 & -0.0009 & 0.801 & 0.006 & -0.006 \\
7.5 & 0.206 & 0.0006 & -0.0006 & 0.783 & 0.005 & -0.005 \\
8 & 0.170 & 0.0005 & -0.0005 & 0.777 & 0.005 & -0.005 \\
8.5 & 0.140 & 0.0004 & -0.0004 & 0.761 & 0.006 & -0.006 \\
9 & 0.115 & 0.0004 & -0.0004 & 0.751 & 0.008 & -0.008 \\
9.5 & 0.0949 & 0.0004 & -0.0004 & 0.749 & 0.01 & -0.01 \\
10 & 0.0784 & 0.0005 & -0.0005 & 0.753 & 0.02 & -0.02 \\
\hline & \multicolumn{7}{|c|}{ LL+ +} & \multicolumn{4}{|c|}{ NLL+ +} \\
$Y$ & MC0 & MC+ + & MC- & MC0 & MC+ & MC- \\
\hline 6 & 0.566 & 0.004 & -0.004 & 0.940 & 0.01 & -0.01 \\
6.5 & 0.514 & 0.002 & -0.002 & 0.919 & 0.009 & -0.009 \\
7 & 0.465 & 0.002 & -0.002 & 0.902 & 0.007 & -0.007 \\
7.5 & 0.421 & 0.001 & -0.001 & 0.893 & 0.007 & -0.007 \\
8 & 0.381 & 0.001 & -0.001 & 0.901 & 0.008 & -0.008 \\
8.5 & 0.344 & 0.001 & -0.001 & 0.895 & 0.009 & -0.009 \\
9 & 0.311 & 0.001 & -0.001 & 0.898 & 0.01 & -0.01 \\
9.5 & 0.280 & 0.001 & -0.001 & 0.908 & 0.02 & -0.02 \\
10 & 0.253 & 0.002 & -0.002 & 0.908 & 0.04 & -0.04 \\
\hline
\end{tabular}

Table 5. Tabled values for figure 6 . 


\begin{tabular}{|l|l|l|l|l|l|l|}
\hline & \multicolumn{3}{|c|}{ LL } & \multicolumn{3}{c|}{ NLL } \\
$Y$ & $\mu=35$ & $\mu=70$ & $\mu=17.5$ & $\mu=35$ & $\mu=70$ & $\mu=17.5$ \\
\hline 6 & 0.377 & 0.1 & -0.1 & 0.851 & -0.06 & 0.07 \\
6.5 & 0.308 & 0.1 & -0.09 & 0.824 & -0.07 & 0.08 \\
7 & 0.252 & 0.09 & -0.08 & 0.801 & -0.07 & 0.09 \\
7.5 & 0.206 & 0.08 & -0.07 & 0.783 & -0.08 & 0.1 \\
8 & 0.170 & 0.07 & -0.06 & 0.777 & -0.1 & 0.1 \\
8.5 & 0.140 & 0.06 & -0.05 & 0.761 & -0.1 & 0.1 \\
9 & 0.115 & 0.05 & -0.04 & 0.751 & -0.1 & 0.1 \\
9.5 & 0.0949 & 0.05 & -0.04 & 0.749 & -0.1 & 0.1 \\
10 & 0.0784 & 0.04 & -0.03 & 0.753 & -0.1 & 0.07 \\
\hline & \multicolumn{5}{|c|}{ LL+ +} & \multicolumn{3}{|c|}{ NLL+ } \\
$Y$ & $\mu=35$ & $\mu=70$ & $\mu=17.5$ & $\mu=35$ & $\mu=70$ & $\mu=17.5$ \\
\hline 6 & 0.566 & 0.02 & -0.02 & 0.940 & -0.06 & 0.07 \\
6.5 & 0.514 & 0.02 & -0.02 & 0.919 & -0.07 & 0.09 \\
7 & 0.465 & 0.02 & -0.02 & 0.902 & -0.08 & 0.1 \\
7.5 & 0.421 & 0.02 & -0.02 & 0.893 & -0.09 & 0.1 \\
8 & 0.381 & 0.02 & -0.02 & 0.901 & -0.1 & 0.2 \\
8.5 & 0.344 & 0.02 & -0.02 & 0.895 & -0.1 & 0.1 \\
9 & 0.311 & 0.02 & -0.02 & 0.898 & -0.1 & 0.09 \\
9.5 & 0.280 & 0.02 & -0.02 & 0.908 & -0.2 & -0.05 \\
10 & 0.253 & 0.02 & -0.01 & 0.908 & -0.1 & -0.3 \\
\hline
\end{tabular}

Table 6. Tabled values for figures 7

\begin{tabular}{|l|l|l|l|l|l|l|}
\hline & \multicolumn{5}{|c|}{ LL } & \multicolumn{3}{c|}{ NLL } \\
$Y$ & $\sqrt{s_{0}}=35$ & $\sqrt{s_{0}}=70$ & $\sqrt{s_{0}}=17.5$ & $\sqrt{s_{0}}=35$ & $\sqrt{s_{0}}=70$ & $\sqrt{s_{0}}=17.5$ \\
\hline 6 & 0.377 & 0.1 & -0.09 & 0.851 & -0.02 & 0.09 \\
6.5 & 0.308 & 0.1 & -0.07 & 0.824 & -0.02 & 0.09 \\
7 & 0.252 & 0.08 & -0.06 & 0.801 & -0.02 & 0.09 \\
7.5 & 0.206 & 0.07 & -0.05 & 0.783 & -0.03 & 0.09 \\
8 & 0.170 & 0.05 & -0.04 & 0.777 & -0.03 & 0.09 \\
8.5 & 0.140 & 0.04 & -0.03 & 0.761 & -0.03 & 0.09 \\
9 & 0.115 & 0.04 & -0.03 & 0.751 & -0.03 & 0.08 \\
9.5 & 0.0949 & 0.03 & -0.02 & 0.749 & -0.02 & 0.07 \\
10 & 0.0784 & 0.02 & -0.02 & 0.753 & -0.02 & 0.05 \\
\hline & \multicolumn{7}{|c|}{ LL+ +} & & \multicolumn{3}{|c|}{ NLL+ } \\
$Y$ & $\sqrt{s_{0}}=35$ & $\sqrt{s_{0}}=70$ & $\sqrt{s_{0}}=17.5$ & $\sqrt{s_{0}}=35$ & $\sqrt{s_{0}}=70$ & $\sqrt{s_{0}}=17.5$ \\
\hline 6 & 0.566 & 0.08 & -0.07 & 0.940 & -0.04 & 0.1 \\
6.5 & 0.514 & 0.07 & -0.07 & 0.919 & -0.04 & 0.1 \\
7 & 0.465 & 0.07 & -0.06 & 0.902 & -0.04 & 0.1 \\
7.5 & 0.421 & 0.06 & -0.06 & 0.893 & -0.05 & 0.1 \\
8 & 0.381 & 0.06 & -0.05 & 0.901 & -0.05 & 0.1 \\
8.5 & 0.344 & 0.05 & -0.05 & 0.895 & -0.05 & 0.1 \\
9 & 0.311 & 0.05 & -0.04 & 0.898 & -0.05 & 0.09 \\
9.5 & 0.280 & 0.04 & -0.04 & 0.908 & -0.05 & 0.05 \\
10 & 0.253 & 0.04 & -0.03 & 0.908 & -0.03 & -0.04 \\
\hline
\end{tabular}

Table 7. Tabled values for figure 8 . 


\begin{tabular}{|l|l|l|l|l|l|l|}
\hline & \multicolumn{3}{|c|}{ NLL } & \multicolumn{3}{c|}{ NLL+ } \\
$Y$ & PDF0 & PDF + & PDF- & PDF0 & PDF+ & PDF- \\
\hline 6 & 0.851 & -0.006 & 0.002 & 0.940 & -0.007 & 0.003 \\
6.5 & 0.824 & -0.006 & 0.003 & 0.919 & -0.007 & 0.004 \\
7 & 0.801 & -0.005 & 0.004 & 0.902 & -0.007 & 0.005 \\
7.5 & 0.783 & -0.005 & 0.003 & 0.893 & -0.006 & 0.004 \\
8 & 0.777 & -0.005 & 0.003 & 0.901 & -0.007 & 0.005 \\
8.5 & 0.761 & -0.005 & 0.004 & 0.895 & -0.007 & 0.005 \\
9 & 0.751 & -0.005 & 0.003 & 0.898 & -0.007 & 0.004 \\
9.5 & 0.749 & -0.006 & 0.003 & 0.908 & -0.008 & 0.004 \\
10 & 0.753 & -0.007 & 0.004 & 0.908 & -0.006 & 0.003 \\
\hline
\end{tabular}

Table 8. Tabled values for figure 9 .

\begin{tabular}{|l|l|l|l|l|l|l|}
\hline & \multicolumn{3}{|c|}{ LL } & \multicolumn{3}{|c|}{ NLL } \\
$Y$ & MC0 & MC+ & MC- & MC0 & MC+ & MC- \\
\hline 6 & 0.258 & 0.003 & -0.003 & 0.512 & 0.006 & -0.006 \\
6.5 & 0.195 & 0.001 & -0.001 & 0.471 & 0.004 & -0.004 \\
7 & 0.147 & 0.0006 & -0.0006 & 0.434 & 0.003 & -0.003 \\
7.5 & 0.111 & 0.0004 & -0.0004 & 0.404 & 0.002 & -0.002 \\
8 & 0.0836 & 0.0002 & -0.0002 & 0.383 & 0.002 & -0.002 \\
8.5 & 0.0633 & 0.0002 & -0.0002 & 0.358 & 0.003 & -0.003 \\
9 & 0.0481 & 0.0002 & -0.0002 & 0.337 & 0.003 & -0.003 \\
9.5 & 0.0365 & 0.0002 & -0.0002 & 0.324 & 0.005 & -0.005 \\
10 & 0.0278 & 0.0002 & -0.0002 & 0.317 & 0.009 & -0.009 \\
\hline & & LL+ & & \multicolumn{3}{|c|}{ NLL+ } \\
$Y$ & MC0 & MC+ & MC- & MC0 & MC+ & MC- \\
\hline 6 & 0.317 & 0.002 & -0.002 & 0.566 & 0.008 & -0.008 \\
6.5 & 0.264 & 0.001 & -0.001 & 0.525 & 0.005 & -0.005 \\
7 & 0.220 & 0.0008 & -0.0008 & 0.489 & 0.004 & -0.004 \\
7.5 & 0.183 & 0.0005 & -0.0005 & 0.461 & 0.003 & -0.003 \\
8 & 0.152 & 0.0004 & -0.0004 & 0.445 & 0.004 & -0.004 \\
8.5 & 0.126 & 0.0004 & -0.0004 & 0.421 & 0.004 & -0.004 \\
9 & 0.105 & 0.0004 & -0.0004 & 0.403 & 0.006 & -0.006 \\
9.5 & 0.0870 & 0.0004 & -0.0004 & 0.393 & 0.008 & -0.008 \\
10 & 0.0721 & 0.0005 & -0.0005 & 0.382 & 0.02 & -0.02 \\
\hline
\end{tabular}

Table 9. Tabled values for figure 10. 


\begin{tabular}{|l|l|l|l|l|l|l|}
\hline & \multicolumn{3}{|c|}{ LL } & \multicolumn{3}{c|}{ NLL } \\
$Y$ & $\mu=35$ & $\mu=70$ & $\mu=17.5$ & $\mu=35$ & $\mu=70$ & $\mu=17.5$ \\
\hline 6 & 0.258 & 0.1 & -0.1 & 0.512 & -0.02 & 0.03 \\
6.5 & 0.195 & 0.1 & -0.08 & 0.471 & -0.03 & 0.04 \\
7 & 0.147 & 0.08 & -0.06 & 0.434 & -0.03 & 0.05 \\
7.5 & 0.111 & 0.07 & -0.05 & 0.404 & -0.04 & 0.06 \\
8 & 0.0836 & 0.05 & -0.04 & 0.383 & -0.04 & 0.08 \\
8.5 & 0.0633 & 0.04 & -0.03 & 0.358 & -0.05 & 0.08 \\
9 & 0.0481 & 0.03 & -0.02 & 0.337 & -0.05 & 0.09 \\
9.5 & 0.0365 & 0.03 & -0.02 & 0.324 & -0.05 & 0.09 \\
10 & 0.0278 & 0.02 & -0.01 & 0.317 & -0.06 & 0.07 \\
\hline & \multicolumn{5}{|c|}{ LL+ } & \multicolumn{5}{|c|}{ NLL+ } \\
$Y$ & $\mu=35$ & $\mu=70$ & $\mu=17.5$ & $\mu=35$ & $\mu=70$ & $\mu=17.5$ \\
\hline 6 & 0.317 & 0.03 & -0.02 & 0.566 & -0.02 & 0.04 \\
6.5 & 0.264 & 0.03 & -0.02 & 0.525 & -0.02 & 0.05 \\
7 & 0.220 & 0.02 & -0.02 & 0.489 & -0.03 & 0.06 \\
7.5 & 0.183 & 0.02 & -0.02 & 0.461 & -0.04 & 0.08 \\
8 & 0.152 & 0.02 & -0.01 & 0.445 & -0.05 & 0.1 \\
8.5 & 0.126 & 0.02 & -0.01 & 0.421 & -0.06 & 0.09 \\
9 & 0.105 & 0.01 & -0.01 & 0.403 & -0.06 & 0.07 \\
9.5 & 0.0870 & 0.01 & -0.008 & 0.393 & -0.07 & 0.01 \\
10 & 0.0721 & 0.01 & -0.007 & 0.382 & -0.07 & -0.09 \\
\hline
\end{tabular}

Table 10. Tabled values for figure 11.

\begin{tabular}{|l|l|l|l|l|l|l|}
\hline & \multicolumn{3}{|c|}{ LL } & \multicolumn{3}{c|}{ NLL } \\
$Y$ & $\sqrt{s_{0}}=35$ & $\sqrt{s_{0}}=70$ & $\sqrt{s_{0}}=17.5$ & $\sqrt{s_{0}}=35$ & $\sqrt{s_{0}}=70$ & $\sqrt{s_{0}}=17.5$ \\
\hline 6 & 0.258 & 0.1 & -0.08 & 0.512 & -0.002 & 0.08 \\
6.5 & 0.195 & 0.09 & -0.06 & 0.471 & -0.005 & 0.07 \\
7 & 0.147 & 0.07 & -0.05 & 0.434 & -0.005 & 0.07 \\
7.5 & 0.111 & 0.05 & -0.04 & 0.404 & -0.005 & 0.06 \\
8 & 0.0836 & 0.04 & -0.03 & 0.383 & -0.004 & 0.06 \\
8.5 & 0.0633 & 0.03 & -0.02 & 0.358 & -0.003 & 0.05 \\
9 & 0.0481 & 0.02 & -0.02 & 0.337 & -0.002 & 0.05 \\
9.5 & 0.0365 & 0.02 & -0.01 & 0.324 & -0.001 & 0.04 \\
10 & 0.0278 & 0.01 & -0.009 & 0.317 & 0.001 & 0.03 \\
\hline & \multicolumn{2}{|c|}{ LL+ +} & & \multicolumn{3}{|c|}{ NLL+ } \\
$Y$ & $\sqrt{s_{0}}=35$ & $\sqrt{s_{0}}=70$ & $\sqrt{s_{0}}=17.5$ & $\sqrt{s_{0}}=35$ & $\sqrt{s_{0}}=70$ & $\sqrt{s_{0}}=17.5$ \\
\hline 6 & 0.317 & 0.09 & -0.07 & 0.566 & -0.009 & 0.09 \\
6.5 & 0.264 & 0.07 & -0.06 & 0.525 & -0.01 & 0.09 \\
7 & 0.220 & 0.06 & -0.05 & 0.489 & -0.01 & 0.09 \\
7.5 & 0.183 & 0.05 & -0.04 & 0.461 & -0.01 & 0.08 \\
8 & 0.152 & 0.04 & -0.03 & 0.445 & -0.02 & 0.08 \\
8.5 & 0.126 & 0.04 & -0.03 & 0.421 & -0.02 & 0.07 \\
9 & 0.105 & 0.03 & -0.02 & 0.403 & -0.01 & 0.06 \\
9.5 & 0.0870 & 0.03 & -0.02 & 0.393 & -0.01 & 0.03 \\
10 & 0.0721 & 0.02 & -0.02 & 0.382 & -0.001 & -0.007 \\
\hline
\end{tabular}

Table 11. Tabled values for figure 12. 


\begin{tabular}{|c|c|c|c|c|c|c|}
\hline \multirow[b]{2}{*}{$Y$} & \multicolumn{3}{|c|}{$\begin{array}{l}\mathrm{LL} \\
\end{array}$} & \multicolumn{3}{|c|}{ NLL } \\
\hline & $\mathrm{MC0}$ & $\mathrm{MC}+$ & MC- & $\mathrm{MC} 0$ & $\mathrm{MC}+$ & MC- \\
\hline 6 & 0.686 & 0.009 & -0.009 & 0.602 & 0.006 & -0.006 \\
\hline 6.5 & 0.632 & 0.005 & -0.005 & 0.571 & 0.004 & -0.004 \\
\hline 7 & 0.582 & 0.003 & -0.003 & 0.542 & 0.003 & -0.003 \\
\hline 7.5 & 0.536 & 0.002 & -0.002 & 0.516 & 0.002 & -0.002 \\
\hline 8 & 0.493 & 0.002 & -0.002 & 0.493 & 0.002 & -0.002 \\
\hline 8.5 & 0.454 & 0.002 & -0.002 & 0.470 & 0.002 & -0.002 \\
\hline 9 & 0.418 & 0.002 & -0.002 & 0.449 & 0.002 & -0.002 \\
\hline 9.5 & 0.385 & 0.002 & -0.002 & 0.432 & 0.003 & -0.003 \\
\hline 10 & 0.354 & 0.003 & -0.003 & 0.421 & 0.005 & -0.005 \\
\hline & & LL+ & & & & \\
\hline$Y$ & MC0 & $\mathrm{MC}+$ & MC- & & & \\
\hline 6 & 0.560 & 0.005 & -0.005 & & & \\
\hline 6.5 & 0.515 & 0.003 & -0.003 & & & \\
\hline 7 & 0.473 & 0.002 & -0.002 & & & \\
\hline 7.5 & 0.435 & 0.002 & -0.002 & & & \\
\hline 8 & 0.400 & 0.002 & -0.002 & & & \\
\hline 8.5 & 0.368 & 0.002 & -0.002 & & & \\
\hline 9 & 0.338 & 0.002 & -0.002 & & & \\
\hline 9.5 & 0.310 & 0.002 & -0.002 & & & \\
\hline 10 & 0.285 & 0.003 & -0.003 & & & \\
\hline
\end{tabular}

Table 12. Tabled values for figure 13 .

\begin{tabular}{|l|l|l|l|l|l|l|}
\hline & \multicolumn{3}{|c|}{ LL } & \multicolumn{3}{c|}{ NLL } \\
$Y$ & $\mu=35$ & $\mu=70$ & $\mu=17.5$ & $\mu=35$ & $\mu=70$ & $\mu=17.5$ \\
\hline 6 & 0.686 & 0.07 & -0.09 & 0.602 & 0.02 & -0.007 \\
6.5 & 0.632 & 0.08 & -0.09 & 0.571 & 0.02 & -0.004 \\
7 & 0.582 & 0.08 & -0.09 & 0.542 & 0.01 & 0.0009 \\
7.5 & 0.536 & 0.08 & -0.09 & 0.516 & 0.01 & 0.007 \\
8 & 0.493 & 0.08 & -0.08 & 0.493 & 0.007 & 0.01 \\
8.5 & 0.454 & 0.08 & -0.08 & 0.470 & 0.004 & 0.02 \\
9 & 0.418 & 0.07 & -0.08 & 0.449 & 0.0007 & 0.03 \\
9.5 & 0.385 & 0.07 & -0.08 & 0.432 & -0.003 & 0.04 \\
10 & 0.354 & 0.07 & -0.07 & 0.421 & -0.009 & 0.05 \\
\hline & \multicolumn{7}{|c|}{ LL+ +} & & & \\
$Y$ & $\mu=35$ & $\mu=70$ & $\mu=17.5$ & & & \\
\hline 6 & 0.560 & 0.03 & -0.02 & & & \\
6.5 & 0.515 & 0.03 & -0.02 & & \\
7 & 0.473 & 0.03 & -0.02 & & \\
7.5 & 0.435 & 0.03 & -0.02 & & \\
8 & 0.400 & 0.02 & -0.02 & & \\
8.5 & 0.368 & 0.02 & -0.02 & & \\
9 & 0.338 & 0.02 & -0.01 & -0.01 & & \\
9.5 & 0.310 & 0.02 & -0.02 & \\
10 & 0.285 & 0.02 & -0.01 &
\end{tabular}

Table 13. Tabled values for figure 14 . 


\begin{tabular}{|c|c|c|c|c|c|c|}
\hline & \multicolumn{3}{|c|}{ LL } & \multicolumn{3}{|c|}{ NLL } \\
\hline$Y$ & $\sqrt{s_{0}}=35$ & $\sqrt{s_{0}}=70$ & $\sqrt{s_{0}}=17.5$ & $\sqrt{s_{0}}=35$ & $\sqrt{s_{0}}=70$ & $\sqrt{s_{0}}=17.5$ \\
\hline 6 & 0.686 & 0.08 & -0.07 & 0.602 & 0.01 & 0.03 \\
\hline 6.5 & 0.632 & 0.08 & -0.07 & 0.571 & 0.01 & 0.02 \\
\hline 7 & 0.582 & 0.07 & -0.06 & 0.542 & 0.01 & 0.02 \\
\hline 7.5 & 0.536 & 0.07 & -0.06 & 0.516 & 0.01 & 0.02 \\
\hline 8 & 0.493 & 0.06 & -0.05 & 0.493 & 0.01 & 0.02 \\
\hline 8.5 & 0.454 & 0.06 & -0.05 & 0.470 & 0.01 & 0.02 \\
\hline 9 & 0.418 & 0.05 & -0.05 & 0.449 & 0.01 & 0.01 \\
\hline 9.5 & 0.385 & 0.05 & -0.04 & 0.432 & 0.01 & 0.01 \\
\hline 10 & 0.354 & 0.04 & -0.04 & 0.421 & 0.01 & 0.01 \\
\hline & & LL+ & & & & \\
\hline$Y$ & $\sqrt{s_{0}}=35$ & $\sqrt{s_{0}}=70$ & $\sqrt{s_{0}}=17.5$ & & & \\
\hline 6 & 0.560 & 0.07 & -0.06 & & & \\
\hline 6.5 & 0.515 & 0.06 & -0.06 & & & \\
\hline 7 & 0.473 & 0.06 & -0.05 & & & \\
\hline 7.5 & 0.435 & 0.05 & -0.0 & & & \\
\hline 8 & 0.400 & 0.05 & -0.04 & & & \\
\hline 8.5 & 0.368 & 0.05 & -0.04 & & & \\
\hline 9 & 0.338 & 0.04 & -0.04 & & & \\
\hline 9.5 & 0.310 & 0.04 & -0.03 & & & \\
\hline 10 & 0.285 & 0.04 & -0.03 & & & \\
\hline
\end{tabular}

Table 14. Tabled values for figure 15 .

\begin{tabular}{|c|c|c|c|c|c|c|}
\hline \multirow[b]{2}{*}{$Y$} & \multicolumn{3}{|c|}{$\mathrm{LL}$} & \multicolumn{3}{|c|}{ NLL } \\
\hline & MC0 & $\mathrm{MC}+$ & MC- & $\mathrm{MC0}$ & $\mathrm{MC}+$ & MC- \\
\hline 6 & 0.287 & 0.002 & -0.002 & 0.258 & 0.002 & -0.002 \\
\hline 6.5 & 0.316 & 0.002 & -0.002 & 0.310 & 0.002 & -0.002 \\
\hline 7 & 0.257 & 0.0009 & -0.0009 & 0.268 & 0.001 & -0.001 \\
\hline 7.5 & 0.176 & 0.0005 & -0.0005 & 0.192 & 0.0007 & -0.0007 \\
\hline 8 & 0.100 & 0.0003 & -0.0003 & 0.112 & 0.0004 & -0.0004 \\
\hline 8.5 & 0.0420 & 0.0001 & -0.0001 & 0.0480 & 0.0002 & -0.0002 \\
\hline 9 & 0.0154 & 0.00005 & -0.00005 & 0.0178 & 0.00007 & -0.00007 \\
\hline 9.5 & 0.00450 & 0.00002 & -0.00002 & 0.00516 & 0.00003 & -0.00003 \\
\hline 10 & 0.000810 & 0.000005 & -0.000005 & 0.000897 & 0.000008 & -0.000008 \\
\hline \multicolumn{4}{|c|}{$\mathrm{LL}+$} & & & \\
\hline$Y$ & $\mathrm{MC0}$ & $\mathrm{MC}+$ & MC- & & & \\
\hline 6 & 0.240 & 0.001 & -0.001 & & & \\
\hline 6.5 & 0.282 & 0.001 & -0.001 & & & \\
\hline 7 & 0.239 & 0.0008 & -0.0008 & & & \\
\hline 7.5 & 0.170 & 0.0005 & -0.0005 & & & \\
\hline 8 & 0.0996 & 0.0003 & -0.0003 & & & \\
\hline 8.5 & 0.0426 & 0.0001 & -0.0001 & & & \\
\hline 9 & 0.0159 & 0.00005 & -0.00005 & & & \\
\hline 9.5 & 0.00472 & 0.00002 & -0.00002 & & & \\
\hline 10 & 0.000861 & 0.000005 & -0.000005 & & & \\
\hline
\end{tabular}

Table 15. Tabled values for figure 16, 18. 


\begin{tabular}{|l|l|l|l|l|l|l|}
\hline & \multicolumn{3}{|c|}{ LL } & \multicolumn{3}{c|}{ NLL } \\
$Y$ & $\mu=35$ & $\mu=70$ & $\mu=17.5$ & $\mu=35$ & $\mu=70$ & $\mu=17.5$ \\
\hline 6 & 0.287 & -0.01 & 0.02 & 0.258 & -0.008 & 0.001 \\
6.5 & 0.316 & -0.03 & 0.04 & 0.310 & -0.01 & 0.004 \\
7 & 0.257 & -0.03 & 0.05 & 0.268 & -0.01 & 0.006 \\
7.5 & 0.176 & -0.03 & 0.04 & 0.192 & -0.01 & 0.005 \\
8 & 0.100 & -0.02 & 0.03 & 0.112 & -0.009 & 0.003 \\
8.5 & 0.0420 & -0.01 & 0.02 & 0.0480 & -0.004 & 0.001 \\
9 & 0.0154 & -0.004 & 0.007 & 0.0178 & -0.002 & 0.0004 \\
9.5 & 0.00450 & -0.001 & 0.002 & 0.00516 & -0.0005 & 0.00006 \\
10 & 0.000810 & -0.0003 & 0.0005 & 0.000897 & -0.0001 & -0.000007 \\
\hline & \multicolumn{7}{|c|}{ LL+ +} & & & \\
$Y$ & $\mu=35$ & $\mu=70$ & $\mu=17.5$ & & & \\
\hline 6 & 0.240 & -0.05 & 0.08 & & & \\
6.5 & 0.282 & -0.07 & 0.1 & & & \\
7 & 0.239 & -0.06 & 0.1 & & & \\
7.5 & 0.170 & -0.05 & 0.08 & & & \\
8 & 0.0996 & -0.03 & 0.06 & & & \\
8.5 & 0.0426 & -0.01 & 0.03 & & \\
9 & 0.0159 & -0.006 & 0.01 & & \\
9.5 & 0.00472 & -0.002 & 0.003 & & \\
10 & 0.000861 & -0.0003 & 0.0007 &
\end{tabular}

Table 16. Tabled values for left figure of figure 17.

\begin{tabular}{|c|c|c|c|c|c|c|}
\hline & \multicolumn{3}{|c|}{ LL } & \multicolumn{3}{|c|}{ NLL } \\
\hline Y & $\sqrt{s_{0}}=35$ & $\sqrt{s_{0}}=70$ & $\sqrt{s_{0}}=17.5$ & $\sqrt{s_{0}}=35$ & $\sqrt{s_{0}}=70$ & $\sqrt{s_{0}}=17.5$ \\
\hline 6 & 0.287 & 0.09 & -0.05 & 0.258 & 0.004 & 0.02 \\
\hline 6.5 & 0.316 & 0.07 & -0.05 & 0.310 & 0.002 & 0.03 \\
\hline 7 & 0.257 & 0.05 & -0.03 & 0.268 & 0.0008 & 0.02 \\
\hline 7.5 & 0.176 & 0.03 & -0.02 & 0.192 & 0.0005 & 0.01 \\
\hline 8 & 0.100 & 0.01 & -0.009 & 0.112 & 0.0003 & 0.007 \\
\hline 8.5 & 0.0420 & 0.005 & -0.003 & 0.0480 & 0.0002 & 0.003 \\
\hline 9 & 0.0154 & 0.001 & -0.001 & 0.0178 & 0.00007 & 0.001 \\
\hline 9.5 & 0.00450 & 0.0004 & -0.0003 & 0.00516 & 0.00002 & 0.0003 \\
\hline 10 & 0.000810 & 0.00006 & -0.00005 & 0.000897 & 0.000003 & 0.00005 \\
\hline & & LL+ & & & & \\
\hline$Y$ & $\sqrt{s_{0}}=35$ & $\sqrt{s_{0}}=70$ & $\sqrt{s_{0}}=17.5$ & & & \\
\hline 6 & 0.240 & 0.04 & -0.03 & & & \\
\hline 6.5 & 0.282 & 0.04 & -0.03 & & & \\
\hline 7 & 0.239 & 0.03 & -0.02 & & & \\
\hline 7.5 & 0.170 & 0.02 & -0.01 & & & \\
\hline 8 & 0.0996 & 0.008 & -0.007 & & & \\
\hline 8.5 & 0.0426 & 0.003 & -0.003 & & & \\
\hline 9 & 0.0159 & 0.001 & -0.0009 & & & \\
\hline 9.5 & 0.00472 & 0.0003 & -0.0002 & & & \\
\hline 10 & 0.000861 & 0.00005 & -0.00004 & & & \\
\hline
\end{tabular}

Table 17. Tabled values for right figure of figure 17. 


\begin{tabular}{|c|c|c|c|c|c|c|}
\hline \multirow[b]{2}{*}{$Y$} & \multicolumn{3}{|c|}{$\mathrm{LL}$} & \multicolumn{3}{|c|}{ NLL } \\
\hline & PDF0 & $\mathrm{PDF}+$ & PDF- & PDF0 & $\mathrm{PDF}+$ & PDF- \\
\hline 6 & 0.287 & 0.02 & -0.02 & 0.258 & 0.01 & -0.02 \\
\hline 6.5 & 0.316 & 0.02 & -0.02 & 0.310 & 0.02 & -0.02 \\
\hline 7 & 0.257 & 0.02 & -0.02 & 0.268 & 0.02 & -0.02 \\
\hline 7.5 & 0.176 & 0.02 & -0.02 & 0.192 & 0.02 & -0.02 \\
\hline 8 & 0.100 & 0.01 & -0.01 & 0.112 & 0.01 & -0.01 \\
\hline 8.5 & 0.0420 & 0.006 & -0.005 & 0.0480 & 0.007 & -0.006 \\
\hline 9 & 0.0154 & 0.003 & -0.002 & 0.0178 & 0.003 & -0.002 \\
\hline 9.5 & 0.00450 & 0.0009 & -0.0007 & 0.00516 & 0.001 & -0.0008 \\
\hline 10 & 0.000810 & 0.0002 & -0.0001 & 0.000897 & 0.0002 & -0.0002 \\
\hline & \multicolumn{3}{|c|}{$\mathrm{LL}+$} & & & \\
\hline Y & PDF0 & $\mathrm{PDF}+$ & PDF- & & & \\
\hline 6 & 0.240 & 0.01 & -0.02 & & & \\
\hline 6.5 & 0.282 & 0.02 & -0.02 & & & \\
\hline 7 & 0.239 & 0.02 & -0.02 & & & \\
\hline 7.5 & 0.170 & 0.02 & -0.02 & & & \\
\hline 8 & 0.0996 & 0.01 & -0.01 & & & \\
\hline 8.5 & 0.0426 & 0.006 & -0.005 & & & \\
\hline 9 & 0.0159 & 0.003 & -0.002 & & & \\
\hline 9.5 & 0.00472 & 0.0009 & -0.0008 & & & \\
\hline 10 & 0.000861 & 0.0002 & -0.0002 & & & \\
\hline
\end{tabular}

Table 18. Tabled values for left figure of figure 18.

\begin{tabular}{|l|l|l|l|l|l|l|}
\hline & \multicolumn{5}{|c|}{ LL } & \multicolumn{3}{c|}{ NLL } \\
$Y$ & MC0 & MC + & MC- & MC0 & MC + & MC- \\
\hline 6 & 0.197 & 0.002 & -0.002 & 0.155 & 0.001 & -0.001 \\
6.5 & 0.200 & 0.001 & -0.001 & 0.177 & 0.0008 & -0.0008 \\
7 & 0.149 & 0.0006 & -0.0006 & 0.145 & 0.0005 & -0.0005 \\
7.5 & 0.0942 & 0.0003 & -0.0003 & 0.0991 & 0.0003 & -0.0003 \\
8 & 0.0495 & 0.0001 & -0.0001 & 0.0554 & 0.0001 & -0.0001 \\
8.5 & 0.0191 & 0.00006 & -0.00006 & 0.0226 & 0.00006 & -0.00006 \\
9 & 0.00644 & 0.00002 & -0.00002 & 0.00798 & 0.00002 & -0.00002 \\
9.5 & 0.00173 & 0.000007 & -0.000007 & 0.00223 & 0.000008 & -0.000008 \\
10 & 0.000287 & 0.000002 & -0.000002 & 0.000377 & 0.000002 & -0.000002 \\
\hline & \multicolumn{7}{|c|}{ LL+ +} & & & \\
$Y$ & MC0 & MC+ + & MC- & & & \\
\hline 6 & 0.134 & 0.0009 & -0.0009 & & & \\
6.5 & 0.145 & 0.0006 & -0.0006 & & & \\
7 & 0.113 & 0.0004 & -0.0004 & & & \\
7.5 & 0.0738 & 0.0002 & -0.0002 & & & \\
8 & 0.0398 & 0.0001 & -0.0001 & & & \\
8.5 & 0.0156 & 0.00005 & -0.00005 & & & \\
9 & 0.00537 & 0.00002 & -0.00002 & & & \\
9.5 & 0.00146 & 0.000006 & -0.000006 & & \\
10 & 0.000245 & 0.00002 & -0.00002 &
\end{tabular}

Table 19. Tabled values for figure 19, 21. 


\begin{tabular}{|l|l|l|l|l|l|l|}
\hline & \multicolumn{3}{|c|}{ LL } & \multicolumn{3}{c|}{ NLL } \\
$Y$ & $\mu=35$ & $\mu=70$ & $\mu=17.5$ & $\mu=35$ & $\mu=70$ & $\mu=17.5$ \\
\hline 6 & 0.197 & 0.01 & -0.01 & 0.155 & 0.0002 & -0.001 \\
6.5 & 0.200 & 0.006 & -0.004 & 0.177 & -0.003 & 0.001 \\
7 & 0.149 & -0.001 & 0.002 & 0.145 & -0.004 & 0.003 \\
7.5 & 0.0942 & -0.004 & 0.005 & 0.0991 & -0.005 & 0.004 \\
8 & 0.0495 & -0.004 & 0.004 & 0.0554 & -0.004 & 0.003 \\
8.5 & 0.0191 & -0.002 & 0.002 & 0.0226 & -0.002 & 0.002 \\
9 & 0.00644 & -0.0008 & 0.001 & 0.00798 & -0.0008 & 0.0007 \\
9.5 & 0.00173 & -0.0003 & 0.0003 & 0.00223 & -0.0002 & 0.0002 \\
10 & 0.000287 & -0.00005 & 0.00007 & 0.000377 & -0.00005 & 0.00004 \\
\hline & \multicolumn{7}{|c|}{ LL+ +} & & & \\
$Y$ & $\mu=35$ & $\mu=70$ & $\mu=17.5$ & & & \\
\hline 6 & 0.134 & -0.02 & 0.04 & & & \\
6.5 & 0.145 & -0.03 & 0.05 & & & \\
7 & 0.113 & -0.03 & 0.04 & & & \\
7.5 & 0.0738 & -0.02 & 0.03 & & & \\
8 & 0.0398 & -0.01 & 0.02 & & & \\
8.5 & 0.0156 & -0.004 & 0.009 & & & \\
9 & 0.00537 & -0.002 & 0.003 & & & \\
9.5 & 0.00146 & -0.0005 & 0.001 & & \\
10 & 0.000245 & -0.00009 & 0.0002 &
\end{tabular}

Table 20. Tabled values for left figure of figure 20.

\begin{tabular}{|l|l|l|l|l|l|l|}
\hline & \multicolumn{5}{|c|}{ LL } & \multicolumn{3}{c|}{ NLL } \\
$Y$ & $\sqrt{s_{0}}=35$ & $\sqrt{s_{0}}=70$ & $\sqrt{s_{0}}=17.5$ & $\sqrt{s_{0}}=35$ & $\sqrt{s_{0}}=70$ & $\sqrt{s_{0}}=17.5$ \\
\hline 6 & 0.197 & 0.09 & -0.05 & 0.155 & 0.006 & 0.02 \\
6.5 & 0.200 & 0.08 & -0.05 & 0.177 & 0.004 & 0.02 \\
7 & 0.149 & 0.05 & -0.03 & 0.145 & 0.003 & 0.02 \\
7.5 & 0.0942 & 0.03 & -0.02 & 0.0991 & 0.002 & 0.01 \\
8 & 0.0495 & 0.01 & -0.009 & 0.0554 & 0.001 & 0.006 \\
8.5 & 0.0191 & 0.005 & -0.003 & 0.0226 & 0.0007 & 0.002 \\
9 & 0.00644 & 0.001 & -0.001 & 0.00798 & 0.0003 & 0.0007 \\
9.5 & 0.00173 & 0.0004 & -0.0003 & 0.00223 & 0.00008 & 0.0002 \\
10 & 0.000287 & 0.00006 & -0.00005 & 0.000377 & 0.00001 & 0.00003 \\
\hline & \multicolumn{7}{|c|}{ LL+ +} & & & \\
$Y$ & $\sqrt{s_{0}}=35$ & $\sqrt{s_{0}}=70$ & $\sqrt{s_{0}}=17.5$ & & & \\
\hline 6 & 0.134 & 0.04 & -0.03 & & & \\
6.5 & 0.145 & 0.04 & -0.03 & & & \\
7 & 0.113 & 0.03 & -0.02 & & & \\
7.5 & 0.0738 & 0.02 & -0.01 & & & \\
8 & 0.0398 & 0.009 & -0.007 & & & \\
8.5 & 0.0156 & 0.003 & -0.003 & & \\
9 & 0.00537 & 0.001 & -0.0009 & & & \\
9.5 & 0.00146 & 0.0003 & -0.0002 & & \\
10 & 0.000245 & 0.00005 & -0.00004 &
\end{tabular}

Table 21. Tabled values for right figure of figure 20 . 


\begin{tabular}{|c|c|c|c|c|c|c|}
\hline \multirow[b]{2}{*}{ Y } & \multicolumn{3}{|c|}{ LL } & \multicolumn{3}{|c|}{ NLL } \\
\hline & PDF0 & $\mathrm{PDF}+$ & PDF- & PDF0 & $\mathrm{PDF}+$ & PDF- \\
\hline 6 & 0.197 & 0.01 & -0.01 & 0.155 & 0.007 & -0.01 \\
\hline 6.5 & 0.200 & 0.01 & -0.01 & 0.177 & 0.01 & -0.01 \\
\hline 7 & 0.149 & 0.01 & -0.01 & 0.145 & 0.01 & -0.01 \\
\hline 7.5 & 0.0942 & 0.009 & -0.009 & 0.0991 & 0.009 & -0.009 \\
\hline 8 & 0.0495 & 0.006 & -0.006 & 0.0554 & 0.006 & -0.006 \\
\hline 8.5 & 0.0191 & 0.003 & -0.002 & 0.0226 & 0.003 & -0.003 \\
\hline 9 & 0.00644 & 0.001 & -0.0009 & 0.00798 & 0.001 & -0.001 \\
\hline 9.5 & 0.00173 & 0.0003 & -0.0003 & 0.00223 & 0.0004 & -0.0003 \\
\hline 10 & 0.000287 & 0.00007 & -0.00005 & 0.000377 & 0.00009 & -0.00007 \\
\hline & & LL+ & & & & \\
\hline$Y$ & PDF0 & $\mathrm{PDF}+$ & PDF- & & & \\
\hline 6 & 0.134 & 0.04 & -0.03 & & & \\
\hline 6.5 & 0.145 & 0.04 & -0.03 & & & \\
\hline 7 & 0.113 & 0.03 & -0.02 & & & \\
\hline 7.5 & 0.0738 & 0.02 & -0.01 & & & \\
\hline 8 & 0.0398 & 0.009 & -0.007 & & & \\
\hline 8.5 & 0.0156 & 0.003 & -0.003 & & & \\
\hline 9 & 0.00537 & 0.001 & -0.0009 & & & \\
\hline 9.5 & 0.00146 & 0.0003 & -0.0002 & & & \\
\hline 10 & 0.000245 & 0.00005 & -0.00004 & & & \\
\hline
\end{tabular}

Table 22. Tabled values for left figure of figure 21 .

\begin{tabular}{|l|l|l|l|l|}
\hline & LL & \multicolumn{3}{|c|}{ NLL } \\
$Y$ & & MC0 & MC + & MC- \\
\hline 6 & 0.162 & 0.0699 & 0.0006 & -0.0006 \\
6.5 & 0.200 & 0.0806 & 0.0005 & -0.0005 \\
7 & 0.179 & 0.0651 & 0.0003 & -0.0003 \\
7.5 & 0.128 & 0.0405 & 0.0002 & -0.0002 \\
8 & 0.0689 & 0.0182 & 0.0001 & -0.0001 \\
8.5 & 0.0288 & 0.00638 & 0.00005 & -0.00005 \\
9 & 0.00973 & 0.00173 & 0.00002 & -0.00002 \\
9.5 & 0.00227 & 0.000305 & 0.00007 & -0.000007 \\
10 & 0.000258 & 0.0000267 & 0.000001 & -0.000001 \\
\hline & LL+ & \multicolumn{4}{|c|}{ NLL+ } \\
$Y$ & & MC0 & MC + & MC- \\
\hline 6 & 0.0940 & 0.0643 & 0.0006 & -0.0006 \\
6.5 & 0.113 & 0.0736 & 0.0005 & -0.0005 \\
7 & 0.0966 & 0.0589 & 0.0004 & -0.0004 \\
7.5 & 0.0654 & 0.0362 & 0.0002 & -0.0002 \\
8 & 0.0332 & 0.0160 & 0.0001 & -0.0001 \\
8.5 & 0.0131 & 0.00553 & 0.00006 & -0.00006 \\
9 & 0.00413 & 0.00148 & 0.00003 & -0.00003 \\
9.5 & 0.000900 & 0.000260 & 0.000009 & -0.000009 \\
10 & 0.0000959 & 0.0000253 & 0.000002 & -0.000002 \\
\hline
\end{tabular}

Table 23. Tabled values for figure 22 . 


\begin{tabular}{|l|l|l|l|l|l|l|}
\hline & \multicolumn{3}{|c|}{$\mathrm{LL}$} & \multicolumn{3}{|c|}{ NLL } \\
$Y$ & $\mu=50$ & $\mu=100$ & $\mu=25$ & $\mu=50$ & $\mu=100$ & $\mu=25$ \\
\hline 6 & 0.162 & -0.04 & 0.08 & 0.0699 & 0.002 & 0.01 \\
6.5 & 0.200 & -0.06 & 0.1 & 0.0806 & 0.003 & 0.003 \\
7 & 0.179 & -0.06 & 0.1 & 0.0651 & 0.002 & -0.001 \\
7.5 & 0.128 & -0.05 & 0.1 & 0.0405 & 0.002 & -0.003 \\
8 & 0.0689 & -0.03 & 0.07 & 0.0182 & 0.001 & -0.002 \\
8.5 & 0.0288 & -0.01 & 0.03 & 0.00638 & 0.0004 & -0.0009 \\
9 & 0.00973 & -0.005 & 0.01 & 0.00173 & 0.0001 & -0.0002 \\
9.5 & 0.00227 & -0.001 & 0.003 & 0.000305 & 0.00003 & -0.00003 \\
10 & 0.000258 & -0.0001 & 0.0004 & 0.0000267 & 0.0000009 & 0.000006 \\
\hline & \multicolumn{7}{|c|}{$\mathrm{LL}+$} & & \multicolumn{3}{|c|}{ NLL+ } \\
$Y$ & $\mu=50$ & $\mu=100$ & $\mu=25$ & $\mu=50$ & $\mu=100$ & $\mu=25$ \\
\hline 6 & 0.0940 & -0.02 & 0.04 & 0.0643 & 0.002 & 0.01 \\
6.5 & 0.113 & -0.03 & 0.05 & 0.0736 & 0.002 & 0.003 \\
7 & 0.0966 & -0.03 & 0.05 & 0.0589 & 0.001 & -0.002 \\
7.5 & 0.0654 & -0.02 & 0.04 & 0.0362 & 0.001 & -0.003 \\
8 & 0.0332 & -0.01 & 0.02 & 0.0160 & 0.0009 & -0.002 \\
8.5 & 0.0131 & -0.005 & 0.009 & 0.00553 & 0.0004 & -0.0006 \\
9 & 0.00413 & -0.002 & 0.003 & 0.00148 & 0.0001 & 0.00001 \\
9.5 & 0.000900 & -0.0004 & 0.0008 & 0.000260 & 0.00002 & 0.0001 \\
10 & 0.0000959 & -0.00004 & 0.00009 & 0.0000253 & -0.000002 & 0.00004 \\
\hline
\end{tabular}

Table 24. Tabled values for left figure of figure 23 .

\begin{tabular}{|l|l|l|l|l|l|l|}
\hline & \multicolumn{3}{|c|}{ LL } & \multicolumn{3}{c|}{ NLL } \\
$Y$ & $\sqrt{s_{0}}=50$ & $\sqrt{s_{0}}=100$ & $\sqrt{s_{0}}=25$ & $\sqrt{s_{0}}=50$ & $\sqrt{s_{0}}=100$ & $\sqrt{s_{0}}=25$ \\
\hline 6 & 0.162 & 0.005 & 0.009 & 0.0699 & 0.002 & -0.0001 \\
6.5 & 0.200 & -0.006 & 0.02 & 0.0806 & 0.002 & -0.001 \\
7 & 0.179 & -0.01 & 0.02 & 0.0651 & 0.002 & -0.002 \\
7.5 & 0.128 & -0.01 & 0.02 & 0.0405 & 0.002 & -0.001 \\
8 & 0.0689 & -0.008 & 0.01 & 0.0182 & 0.001 & -0.0006 \\
8.5 & 0.0288 & -0.004 & 0.005 & 0.00638 & 0.0004 & -0.0002 \\
9 & 0.00973 & -0.001 & 0.002 & 0.00173 & 0.0001 & -0.00003 \\
9.5 & 0.00227 & -0.0003 & 0.0004 & 0.000305 & 0.00001 & 0.000008 \\
10 & 0.000258 & -0.00004 & 0.00005 & 0.0000267 & -0.0000007 & 0.000003 \\
\hline & & \multicolumn{1}{|c|}{ LL+ +} & & \multicolumn{3}{|c|}{ NLL+ } \\
$Y$ & $\sqrt{s_{0}}=50$ & $\sqrt{s_{0}}=100$ & $\sqrt{s_{0}}=25$ & $\sqrt{s_{0}}=50$ & $\sqrt{s_{0}}=100$ & $\sqrt{s_{0}}=25$ \\
\hline 6 & 0.0940 & 0.003 & 0.0007 & 0.0643 & 0.003 & -0.0005 \\
6.5 & 0.113 & 0.0002 & 0.003 & 0.0736 & 0.003 & -0.002 \\
7 & 0.0966 & -0.002 & 0.004 & 0.0589 & 0.003 & -0.002 \\
7.5 & 0.0654 & -0.002 & 0.004 & 0.0362 & 0.003 & -0.001 \\
8 & 0.0332 & -0.002 & 0.002 & 0.0160 & 0.001 & -0.0006 \\
8.5 & 0.0131 & -0.0008 & 0.001 & 0.00553 & 0.0005 & -0.0001 \\
9 & 0.00413 & -0.0003 & 0.0003 & 0.00148 & 0.0001 & 0.00003 \\
9.5 & 0.000900 & -0.00007 & 0.00008 & 0.000260 & 0.00001 & 0.00003 \\
10 & 0.0000959 & -0.000007 & 0.000009 & 0.0000253 & -0.000003 & 0.000009 \\
\hline
\end{tabular}

Table 25. Tabled values for right figure of figure 23 . 


\begin{tabular}{|l|c|c|c|c|c|c|}
\hline & \multicolumn{3}{|c|}{ LL } & \multicolumn{3}{c|}{ NLL } \\
$Y$ & MC0 & MC+ & MC- & MC0 & MC+ & MC- \\
\hline 6 & 0.435 & 0.004 & -0.004 & 0.886 & 0.009 & -0.009 \\
6.5 & 0.360 & 0.002 & -0.002 & 0.865 & 0.006 & -0.006 \\
7 & 0.298 & 0.001 & -0.001 & 0.849 & 0.005 & -0.005 \\
7.5 & 0.246 & 0.0008 & -0.0008 & 0.842 & 0.005 & -0.005 \\
8 & 0.204 & 0.0006 & -0.0006 & 0.849 & 0.006 & -0.006 \\
8.5 & 0.170 & 0.0005 & -0.0005 & 0.850 & 0.008 & -0.008 \\
9 & 0.141 & 0.0005 & -0.0005 & 0.866 & 0.01 & -0.01 \\
9.5 & 0.118 & 0.0006 & -0.0006 & 0.899 & 0.02 & -0.02 \\
10 & 0.0984 & 0.0008 & -0.0008 & 0.878 & 0.05 & -0.05 \\
\hline & \multicolumn{5}{|c|}{ LL+ } & \multicolumn{3}{|c|}{ NLL+ } \\
$Y$ & MC0 & MC+ + & MC- & MC0 & MC+ & MC- \\
\hline 6 & 0.597 & 0.003 & -0.003 & 0.963 & 0.01 & -0.01 \\
6.5 & 0.545 & 0.002 & -0.002 & 0.948 & 0.008 & -0.008 \\
7 & 0.496 & 0.002 & -0.002 & 0.938 & 0.007 & -0.007 \\
7.5 & 0.451 & 0.001 & -0.001 & 0.942 & 0.007 & -0.007 \\
8 & 0.409 & 0.001 & -0.001 & 0.965 & 0.008 & -0.008 \\
8.5 & 0.371 & 0.001 & -0.001 & 0.980 & 0.01 & -0.01 \\
9 & 0.336 & 0.001 & -0.001 & 1.01 & 0.02 & -0.02 \\
9.5 & 0.305 & 0.001 & -0.001 & 1.05 & 0.04 & -0.04 \\
10 & 0.276 & 0.002 & -0.002 & 0.925 & 0.07 & -0.07 \\
\hline
\end{tabular}

Table 26. Tabled values for figure 24 .

\begin{tabular}{|l|l|l|l|l|l|l|}
\hline & \multicolumn{3}{|c|}{ LL } & \multicolumn{3}{c|}{ NLL } \\
$Y$ & $\mu=50$ & $\mu=100$ & $\mu=25$ & $\mu=50$ & $\mu=100$ & $\mu=25$ \\
\hline 6 & 0.435 & 0.1 & -0.1 & 0.886 & -0.06 & 0.02 \\
6.5 & 0.360 & 0.1 & -0.1 & 0.865 & -0.07 & 0.07 \\
7 & 0.298 & 0.09 & -0.09 & 0.849 & -0.08 & 0.09 \\
7.5 & 0.246 & 0.08 & -0.08 & 0.842 & -0.09 & 0.1 \\
8 & 0.204 & 0.07 & -0.07 & 0.849 & -0.1 & 0.2 \\
8.5 & 0.170 & 0.07 & -0.06 & 0.850 & -0.1 & 0.1 \\
9 & 0.141 & 0.06 & -0.05 & 0.866 & -0.1 & 0.1 \\
9.5 & 0.118 & 0.05 & -0.04 & 0.899 & -0.1 & 0.05 \\
10 & 0.0984 & 0.04 & -0.04 & 0.878 & -0.1 & -0.2 \\
\hline & & LL+ & & \multicolumn{3}{|c|}{ NLL+ } \\
$Y$ & $\mu=50$ & $\mu=100$ & $\mu=25$ & $\mu=50$ & $\mu=100$ & $\mu=25$ \\
\hline 6 & 0.597 & 0.02 & -0.02 & 0.963 & -0.05 & 0.02 \\
6.5 & 0.545 & 0.02 & -0.02 & 0.948 & -0.07 & 0.08 \\
7 & 0.496 & 0.02 & -0.02 & 0.938 & -0.08 & 0.1 \\
7.5 & 0.451 & 0.02 & -0.02 & 0.942 & -0.1 & 0.2 \\
8 & 0.409 & 0.02 & -0.02 & 0.965 & -0.1 & 0.2 \\
8.5 & 0.371 & 0.02 & -0.02 & 0.980 & -0.1 & 0.1 \\
9 & 0.336 & 0.02 & -0.02 & 1.01 & -0.2 & -0.03 \\
9.5 & 0.305 & 0.02 & -0.01 & 1.05 & -0.2 & -0.3 \\
10 & 0.276 & 0.02 & -0.01 & 0.925 & -0.003 & -0.6 \\
\hline
\end{tabular}

Table 27. Tabled values for figure 25 . 


\begin{tabular}{|l|l|l|l|l|l|l|}
\hline & \multicolumn{5}{|c|}{ LL } & \multicolumn{3}{c|}{ NLL } \\
$Y$ & $\sqrt{s_{0}}=50$ & $\sqrt{s_{0}}=100$ & $\sqrt{s_{0}}=25$ & $\sqrt{s_{0}}=50$ & $\sqrt{s_{0}}=100$ & $\sqrt{s_{0}}=25$ \\
\hline 6 & 0.435 & 0.1 & -0.1 & 0.886 & -0.1 & 0.08 \\
6.5 & 0.360 & 0.1 & -0.08 & 0.865 & -0.1 & 0.08 \\
7 & 0.298 & 0.09 & -0.07 & 0.849 & -0.1 & 0.09 \\
7.5 & 0.246 & 0.07 & -0.06 & 0.842 & -0.1 & 0.09 \\
8 & 0.204 & 0.06 & -0.05 & 0.849 & -0.1 & 0.08 \\
8.5 & 0.170 & 0.05 & -0.04 & 0.850 & -0.1 & 0.08 \\
9 & 0.141 & 0.04 & -0.03 & 0.866 & -0.1 & 0.06 \\
9.5 & 0.118 & 0.03 & -0.03 & 0.899 & -0.1 & 0.03 \\
10 & 0.0984 & 0.03 & -0.02 & 0.878 & -0.05 & -0.05 \\
\hline & & \multicolumn{1}{|c|}{ LL+ } & & & \multicolumn{2}{|c|}{ NLL+ } \\
$Y$ & $\sqrt{s_{0}}=50$ & $\sqrt{s_{0}}=100$ & $\sqrt{s_{0}}=25$ & $\sqrt{s_{0}}=50$ & $\sqrt{s_{0}}=100$ & $\sqrt{s_{0}}=25$ \\
\hline 6 & 0.597 & 0.08 & -0.07 & 0.963 & -0.1 & 0.09 \\
6.5 & 0.545 & 0.07 & -0.07 & 0.948 & -0.1 & 0.1 \\
7 & 0.496 & 0.07 & -0.06 & 0.938 & -0.1 & 0.1 \\
7.5 & 0.451 & 0.06 & -0.06 & 0.942 & -0.1 & 0.1 \\
8 & 0.409 & 0.06 & -0.05 & 0.965 & -0.2 & 0.1 \\
8.5 & 0.371 & 0.05 & -0.05 & 0.980 & -0.2 & 0.08 \\
9 & 0.336 & 0.05 & -0.04 & 1.01 & -0.2 & 0.04 \\
9.5 & 0.305 & 0.04 & -0.04 & 1.05 & -0.1 & -0.07 \\
10 & 0.276 & 0.04 & -0.04 & 0.925 & 0.03 & -0.2 \\
\hline
\end{tabular}

Table 28. Tabled values for figure 26 .

\begin{tabular}{|l|l|c|c|c|c|c|}
\hline & \multicolumn{3}{|c|}{ LL } & \multicolumn{3}{c|}{ NLL } \\
$Y$ & MC0 & MC+ & MC- & MC0 & MC+ & MC- \\
\hline 6 & 0.316 & 0.003 & -0.003 & 0.576 & 0.006 & -0.006 \\
6.5 & 0.242 & 0.001 & -0.001 & 0.537 & 0.004 & -0.004 \\
7 & 0.185 & 0.0007 & -0.0007 & 0.504 & 0.003 & -0.003 \\
7.5 & 0.142 & 0.0005 & -0.0005 & 0.481 & 0.003 & -0.003 \\
8 & 0.109 & 0.0003 & -0.0003 & 0.469 & 0.003 & -0.003 \\
8.5 & 0.0838 & 0.0003 & -0.0003 & 0.453 & 0.004 & -0.004 \\
9 & 0.0645 & 0.0002 & -0.0002 & 0.450 & 0.006 & -0.006 \\
9.5 & 0.0498 & 0.0002 & -0.0002 & 0.464 & 0.01 & -0.01 \\
10 & 0.0384 & 0.0003 & -0.0003 & 0.459 & 0.02 & -0.02 \\
\hline & \multicolumn{7}{|c|}{ LL+ +} & \multicolumn{4}{|c|}{ NLL+ } \\
$Y$ & MC0 & MC+ & MC- & MC0 & MC+ & MC- \\
\hline 6 & 0.358 & 0.002 & -0.002 & 0.626 & 0.007 & -0.007 \\
6.5 & 0.302 & 0.001 & -0.001 & 0.589 & 0.005 & -0.005 \\
7 & 0.254 & 0.0008 & -0.0008 & 0.557 & 0.004 & -0.004 \\
7.5 & 0.213 & 0.0006 & -0.0006 & 0.537 & 0.004 & -0.004 \\
8 & 0.179 & 0.0005 & -0.0005 & 0.533 & 0.004 & -0.004 \\
8.5 & 0.150 & 0.0005 & -0.0005 & 0.523 & 0.006 & -0.006 \\
9 & 0.126 & 0.0005 & -0.0005 & 0.527 & 0.009 & -0.009 \\
9.5 & 0.105 & 0.0005 & -0.0005 & 0.543 & 0.02 & -0.02 \\
10 & 0.0880 & 0.0007 & -0.0007 & 0.484 & 0.04 & -0.04 \\
\hline
\end{tabular}

Table 29. Tabled values for figure 27 . 


\begin{tabular}{|l|l|l|l|l|l|l|}
\hline & \multicolumn{3}{|c|}{ LL } & \multicolumn{3}{c|}{ NLL } \\
$Y$ & $\mu=50$ & $\mu=100$ & $\mu=25$ & $\mu=50$ & $\mu=100$ & $\mu=25$ \\
\hline 6 & 0.316 & 0.1 & -0.1 & 0.576 & -0.03 & -0.09 \\
6.5 & 0.242 & 0.1 & -0.09 & 0.537 & -0.03 & -0.01 \\
7 & 0.185 & 0.09 & -0.07 & 0.504 & -0.04 & 0.03 \\
7.5 & 0.142 & 0.07 & -0.06 & 0.481 & -0.05 & 0.06 \\
8 & 0.109 & 0.06 & -0.05 & 0.469 & -0.06 & 0.1 \\
8.5 & 0.0838 & 0.05 & -0.04 & 0.453 & -0.07 & 0.1 \\
9 & 0.0645 & 0.04 & -0.03 & 0.450 & -0.08 & 0.1 \\
9.5 & 0.0498 & 0.03 & -0.02 & 0.464 & -0.09 & 0.09 \\
10 & 0.0384 & 0.03 & -0.02 & 0.459 & -0.08 & -0.05 \\
\hline & & LL+ & & \multicolumn{3}{|c|}{ NLL+ } \\
$Y$ & $\mu=50$ & $\mu=100$ & $\mu=25$ & $\mu=50$ & $\mu=100$ & $\mu=25$ \\
\hline 6 & 0.358 & 0.03 & -0.02 & 0.626 & -0.02 & -0.1 \\
6.5 & 0.302 & 0.03 & -0.02 & 0.589 & -0.03 & -0.01 \\
7 & 0.254 & 0.02 & -0.02 & 0.557 & -0.04 & 0.04 \\
7.5 & 0.213 & 0.02 & -0.02 & 0.537 & -0.05 & 0.08 \\
8 & 0.179 & 0.02 & -0.01 & 0.533 & -0.07 & 0.1 \\
8.5 & 0.150 & 0.02 & -0.01 & 0.523 & -0.08 & 0.1 \\
9 & 0.126 & 0.01 & -0.01 & 0.527 & -0.09 & 0.04 \\
9.5 & 0.105 & 0.01 & -0.009 & 0.543 & -0.1 & -0.1 \\
10 & 0.0880 & 0.01 & -0.007 & 0.484 & -0.03 & -0.3 \\
\hline
\end{tabular}

Table 30. Tabled values for figure 28 .

\begin{tabular}{|c|c|c|c|c|c|c|}
\hline \multirow[b]{2}{*}{ Y } & \multicolumn{3}{|c|}{$\mathrm{LL}$} & \multicolumn{3}{|c|}{ NLL } \\
\hline & $\sqrt{s_{0}}=50$ & $\sqrt{s_{0}}=100$ & $\sqrt{s_{0}}=17.5$ & $\sqrt{s_{0}}=50$ & $\sqrt{s_{0}}=100$ & $\sqrt{s_{0}}=25$ \\
\hline 6 & 0.316 & 0.1 & -0.1 & 0.576 & -0.08 & 0.08 \\
\hline 6.5 & 0.242 & 0.1 & -0.07 & 0.537 & -0.09 & 0.07 \\
\hline 7 & 0.185 & 0.08 & -0.06 & 0.504 & -0.09 & 0.07 \\
\hline 7.5 & 0.142 & 0.06 & -0.04 & 0.481 & -0.09 & 0.07 \\
\hline 8 & 0.109 & 0.05 & -0.03 & 0.469 & -0.08 & 0.03 \\
\hline 8.5 & 0.0838 & 0.04 & -0.03 & 0.453 & -0.08 & 0.01 \\
\hline 9 & 0.0645 & 0.03 & -0.02 & 0.450 & -0.08 & -0.03 \\
\hline 9.5 & 0.0498 & 0.02 & -0.01 & 0.464 & -0.07 & -0.1 \\
\hline 10 & 0.0384 & 0.02 & -0.01 & 0.459 & -0.04 & -0.3 \\
\hline \multirow[b]{2}{*}{ Y } & \multicolumn{3}{|c|}{ LL+ } & \multicolumn{3}{|c|}{ NLL+ } \\
\hline & $\sqrt{s_{0}}=50$ & $\sqrt{s_{0}}=100$ & $\sqrt{s_{0}}=25$ & $\sqrt{s_{0}}=50$ & $\sqrt{s_{0}}=100$ & $\sqrt{s_{0}}=25$ \\
\hline 6 & 0.358 & 0.09 & -0.08 & 0.626 & -0.1 & 0.09 \\
\hline 6.5 & 0.302 & 0.08 & -0.06 & 0.589 & -0.1 & 0.09 \\
\hline 7 & 0.254 & 0.07 & -0.05 & 0.557 & -0.1 & 0.08 \\
\hline 7.5 & 0.213 & 0.06 & -0.05 & 0.537 & -0.1 & 0.08 \\
\hline 8 & 0.179 & 0.05 & -0.04 & 0.533 & -0.1 & 0.04 \\
\hline 8.5 & 0.150 & 0.04 & -0.03 & 0.523 & -0.1 & 0.01 \\
\hline 9 & 0.126 & 0.03 & -0.03 & 0.527 & -0.1 & -0.05 \\
\hline 9.5 & 0.105 & 0.03 & -0.02 & 0.543 & -0.08 & -0.2 \\
\hline 10 & 0.0880 & 0.02 & -0.02 & 0.484 & -0.005 & -0.3 \\
\hline
\end{tabular}

Table 31. Tabled values for figure 29. 


\begin{tabular}{|c|c|c|c|c|c|c|}
\hline \multirow[b]{2}{*}{$Y$} & \multicolumn{3}{|c|}{ LL } & \multicolumn{3}{|c|}{ NLL } \\
\hline & $\mathrm{MC} 0$ & $\mathrm{MC}+$ & MC- & $\mathrm{MC} 0$ & $\mathrm{MC}+$ & MC- \\
\hline 6 & 0.726 & 0.01 & -0.01 & 0.650 & 0.006 & -0.006 \\
\hline 6.5 & 0.673 & 0.005 & -0.005 & 0.621 & 0.004 & -0.004 \\
\hline 7 & 0.623 & 0.003 & -0.003 & 0.594 & 0.003 & -0.003 \\
\hline 7.5 & 0.576 & 0.003 & -0.003 & 0.571 & 0.002 & -0.002 \\
\hline 8 & 0.533 & 0.002 & -0.002 & 0.552 & 0.002 & -0.002 \\
\hline 8.5 & 0.493 & 0.002 & -0.002 & 0.533 & 0.003 & -0.003 \\
\hline 9 & 0.456 & 0.002 & -0.002 & 0.520 & 0.003 & -0.003 \\
\hline 9.5 & 0.422 & 0.003 & -0.003 & 0.516 & 0.004 & -0.004 \\
\hline 10 & 0.390 & 0.004 & -0.004 & 0.523 & 0.008 & -0.008 \\
\hline & & LL+ & & & & \\
\hline$Y$ & $\mathrm{MC} 0$ & $\mathrm{MC}+$ & MC- & & & \\
\hline 6 & 0.599 & 0.005 & -0.005 & & & \\
\hline 6.5 & 0.554 & 0.003 & -0.003 & & & \\
\hline 7 & 0.512 & 0.002 & -0.002 & & & \\
\hline 7.5 & 0.473 & 0.002 & -0.002 & & & \\
\hline 8 & 0.437 & 0.002 & -0.002 & & & \\
\hline 8.5 & 0.404 & 0.002 & -0.002 & & & \\
\hline 9 & 0.373 & 0.002 & -0.002 & & & \\
\hline 9.5 & 0.345 & 0.002 & -0.002 & & & \\
\hline 10 & 0.319 & 0.003 & -0.003 & & & \\
\hline
\end{tabular}

Table 32. Tabled values for figure 30 .

\begin{tabular}{|l|l|l|l|l|l|l|}
\hline & \multicolumn{3}{|c|}{ LL } & \multicolumn{3}{c|}{ NLL } \\
$Y$ & $\mu=50$ & $\mu=100$ & $\mu=25$ & $\mu=50$ & $\mu=100$ & $\mu=25$ \\
\hline 6 & 0.726 & 0.07 & -0.08 & 0.650 & 0.01 & -0.1 \\
6.5 & 0.673 & 0.07 & -0.08 & 0.621 & 0.01 & -0.06 \\
7 & 0.623 & 0.07 & -0.08 & 0.594 & 0.009 & -0.03 \\
7.5 & 0.576 & 0.07 & -0.08 & 0.571 & 0.005 & -0.006 \\
8 & 0.533 & 0.07 & -0.08 & 0.552 & 0.0004 & 0.02 \\
8.5 & 0.493 & 0.07 & -0.08 & 0.533 & -0.004 & 0.04 \\
9 & 0.456 & 0.07 & -0.08 & 0.520 & -0.01 & 0.05 \\
9.5 & 0.422 & 0.07 & -0.07 & 0.516 & -0.02 & 0.07 \\
10 & 0.390 & 0.07 & -0.07 & 0.523 & -0.03 & 0.1 \\
\hline & \multicolumn{7}{|c|}{ LL+ +} & & & \\
$Y$ & $\mu=50$ & $\mu=100$ & $\mu=25$ & & & \\
\hline 6 & 0.599 & 0.03 & -0.02 & & & \\
6.5 & 0.554 & 0.03 & -0.02 & & \\
7 & 0.512 & 0.02 & -0.02 & & \\
7.5 & 0.473 & 0.02 & -0.02 & & \\
8 & 0.437 & 0.02 & -0.02 & & \\
8.5 & 0.404 & 0.02 & -0.01 & & \\
9 & 0.373 & 0.02 & -0.01 & & \\
9.5 & 0.345 & 0.02 & -0.01 & & \\
10 & 0.319 & 0.02 & -0.01 &
\end{tabular}

Table 33. Tabled values for figure 31 . 


\begin{tabular}{|c|c|c|c|c|c|c|}
\hline & \multicolumn{3}{|c|}{ LL } & \multicolumn{3}{|c|}{ NLL } \\
\hline$Y$ & $\sqrt{s_{0}}=50$ & $\sqrt{s_{0}}=100$ & $\sqrt{s_{0}}=17.5$ & $\sqrt{s_{0}}=50$ & $\sqrt{s_{0}}=100$ & $\sqrt{s_{0}}=25$ \\
\hline 6 & 0.726 & 0.07 & -0.07 & 0.650 & -0.03 & 0.02 \\
\hline 6.5 & 0.673 & 0.07 & -0.07 & 0.621 & -0.04 & 0.02 \\
\hline 7 & 0.623 & 0.07 & -0.06 & 0.594 & -0.04 & 0.02 \\
\hline 7.5 & 0.576 & 0.07 & -0.06 & 0.571 & -0.03 & 0.02 \\
\hline 8 & 0.533 & 0.06 & -0.05 & 0.552 & -0.03 & -0.01 \\
\hline 8.5 & 0.493 & 0.06 & -0.05 & 0.533 & -0.03 & -0.03 \\
\hline 9 & 0.456 & 0.05 & -0.05 & 0.520 & -0.03 & -0.07 \\
\hline 9.5 & 0.422 & 0.05 & -0.04 & 0.516 & -0.02 & -0.2 \\
\hline 10 & 0.390 & 0.04 & -0.04 & 0.523 & -0.02 & -0.3 \\
\hline & & LL+ & & & & \\
\hline$Y$ & $\sqrt{s_{0}}=50$ & $\sqrt{s_{0}}=100$ & $\sqrt{s_{0}}=25$ & & & \\
\hline 6 & 0.599 & 0.07 & -0.06 & & & \\
\hline 6.5 & 0.554 & 0.06 & -0.06 & & & \\
\hline 7 & 0.512 & 0.06 & -0.05 & & & \\
\hline 7.5 & 0.473 & 0.05 & -0.05 & & & \\
\hline 8 & 0.437 & 0.05 & -0.05 & & & \\
\hline 8.5 & 0.404 & 0.05 & -0.04 & & & \\
\hline 9 & 0.373 & 0.04 & -0.04 & & & \\
\hline 9.5 & 0.345 & 0.04 & -0.04 & & & \\
\hline 10 & 0.319 & 0.04 & -0.03 & & & \\
\hline
\end{tabular}

Table 34. Tabled values for figure 32 .

\begin{tabular}{|c|c|c|c|c|c|c|}
\hline \multirow[b]{2}{*}{ Y } & \multicolumn{3}{|c|}{ LL } & \multicolumn{3}{|c|}{ NLL } \\
\hline & $\mathrm{MC} 0$ & $\mathrm{MC}+$ & MC- & $\mathrm{MC0}$ & $\mathrm{MC}+$ & MC- \\
\hline 6 & 0.0352 & 0.0003 & -0.0003 & 0.0310 & 0.0002 & -0.0002 \\
\hline 6.5 & 0.0360 & 0.0002 & -0.0002 & 0.0349 & 0.0002 & -0.0002 \\
\hline 7 & 0.0266 & 0.0001 & -0.0001 & 0.0276 & 0.0001 & -0.0001 \\
\hline 7.5 & 0.0157 & 0.00005 & -0.00005 & 0.0170 & 0.00005 & -0.00005 \\
\hline 8 & 0.00704 & 0.00002 & -0.00002 & 0.00773 & 0.00003 & -0.00003 \\
\hline 8.5 & 0.00245 & 0.000008 & -0.000008 & 0.00271 & 0.00001 & -0.00001 \\
\hline 9 & 0.000688 & 0.000003 & -0.000003 & 0.000748 & 0.000004 & -0.000004 \\
\hline 9.5 & 0.000134 & 0.0000006 & -0.0000006 & 0.000137 & 0.000001 & -0.000001 \\
\hline 10 & 0.0000127 & 0.0000001 & -0.0000001 & 0.0000117 & 0.0000002 & -0.0000002 \\
\hline \multicolumn{4}{|c|}{ LL+ } & & & \\
\hline Y & $\mathrm{MC0}$ & $\mathrm{MC}+$ & MC- & & & \\
\hline 6 & 0.0281 & 0.0002 & -0.0002 & & & \\
\hline 6.5 & 0.0308 & 0.0001 & -0.0001 & & & \\
\hline 7 & 0.0239 & 0.00007 & -0.00007 & & & \\
\hline 7.5 & 0.0147 & 0.00004 & -0.00004 & & & \\
\hline 8 & 0.00680 & 0.00002 & -0.00002 & & & \\
\hline 8.5 & 0.00242 & 0.000008 & -0.000008 & & & \\
\hline 9 & 0.000695 & 0.000003 & -0.000003 & & & \\
\hline 9.5 & 0.000137 & 0.0000007 & -0.0000007 & & & \\
\hline 10 & 0.0000132 & 0.0000001 & -0.0000001 & & & \\
\hline
\end{tabular}

Table 35. Tabled values for figure 33 . 


\begin{tabular}{|l|l|l|l|l|l|l|}
\hline & \multicolumn{7}{|c|}{ LL } & \multicolumn{3}{|c|}{ NLL } \\
$Y$ & $\mu=50$ & $\mu=100$ & $\mu=25$ & $\mu=50$ & $\mu=100$ & $\mu=25$ \\
\hline 6 & 0.0352 & -0.001 & 0.003 & 0.0310 & -0.001 & 0.007 \\
6.5 & 0.0360 & -0.003 & 0.006 & 0.0349 & -0.002 & 0.004 \\
7 & 0.0266 & -0.004 & 0.006 & 0.0276 & -0.002 & 0.002 \\
7.5 & 0.0157 & -0.003 & 0.004 & 0.0170 & -0.001 & 0.001 \\
8 & 0.00704 & -0.001 & 0.002 & 0.00773 & -0.0006 & 0.0002 \\
8.5 & 0.00245 & -0.0006 & 0.001 & 0.00271 & -0.0002 & -0.000007 \\
9 & 0.000688 & -0.0002 & 0.0003 & 0.000748 & -0.00007 & -0.00001 \\
9.5 & 0.000134 & -0.00004 & 0.00007 & 0.000137 & -0.00001 & -0.000007 \\
10 & 0.0000127 & -0.000004 & 0.000008 & 0.0000117 & -0.0000009 & -0.000001 \\
\hline & \multicolumn{7}{|c|}{ LL+ } & & & \\
$Y$ & $\mu=50$ & $\mu=100$ & $\mu=25$ & & & \\
\hline 6 & 0.0281 & -0.006 & 0.01 & & & \\
6.5 & 0.0308 & -0.008 & 0.01 & & & \\
7 & 0.0239 & -0.007 & 0.01 & & & \\
7.5 & 0.0147 & -0.004 & 0.008 & & & \\
8 & 0.00680 & -0.002 & 0.004 & & & \\
8.5 & 0.00242 & -0.0008 & 0.002 & & & \\
9 & 0.000695 & -0.0002 & 0.0005 & & & \\
9.5 & 0.000137 & -0.00005 & 0.0001 & & & \\
10 & 0.0000132 & -0.000005 & 0.00001 &
\end{tabular}

Table 36. Tabled values for left figure of figure 34 .

\begin{tabular}{|c|c|c|c|c|c|c|}
\hline & \multicolumn{3}{|c|}{ LL } & \multicolumn{3}{|c|}{ NLL } \\
\hline$Y$ & $\sqrt{s_{0}}=50$ & $\sqrt{s_{0}}=100$ & $\sqrt{s_{0}}=25$ & $\sqrt{s_{0}}=50$ & $\sqrt{s_{0}}=100$ & $\sqrt{s_{0}}=25$ \\
\hline 6 & 0.0352 & 0.01 & -0.007 & 0.0310 & -0.002 & 0.003 \\
\hline 6.5 & 0.0360 & 0.009 & -0.006 & 0.0349 & -0.003 & 0.003 \\
\hline 7 & 0.0266 & 0.005 & -0.004 & 0.0276 & -0.003 & 0.002 \\
\hline 7.5 & 0.0157 & 0.003 & -0.002 & 0.0170 & -0.001 & 0.001 \\
\hline 8 & 0.00704 & 0.001 & -0.0007 & 0.00773 & -0.0006 & 0.0005 \\
\hline 8.5 & 0.00245 & 0.0003 & -0.0002 & 0.00271 & -0.0002 & 0.0002 \\
\hline 9 & 0.000688 & 0.00007 & -0.00005 & 0.000748 & -0.00006 & 0.00004 \\
\hline 9.5 & 0.000134 & 0.00001 & -0.000009 & 0.000137 & -0.00001 & 0.000008 \\
\hline 10 & 0.0000127 & 0.000001 & -0.0000008 & 0.0000117 & -0.0000009 & 0.0000007 \\
\hline & & $\mathrm{LL}+$ & & & & \\
\hline$Y$ & $\sqrt{s_{0}}=50$ & $\sqrt{s_{0}}=100$ & $\sqrt{s_{0}}=25$ & & & \\
\hline 6 & 0.0281 & 0.005 & -0.003 & & & \\
\hline 6.5 & 0.0308 & 0.004 & -0.003 & & & \\
\hline 7 & 0.0239 & 0.003 & -0.002 & & & \\
\hline 7.5 & 0.0147 & 0.001 & -0.001 & & & \\
\hline 8 & 0.00680 & 0.0006 & -0.0005 & & & \\
\hline 8.5 & 0.00242 & 0.0002 & -0.0002 & & & \\
\hline 9 & 0.000695 & 0.00005 & -0.00004 & & & \\
\hline 9.5 & 0.000137 & 0.000009 & -0.000007 & & & \\
\hline 10 & 0.0000132 & 0.0000008 & -0.0000006 & & & \\
\hline
\end{tabular}

Table 37. Tabled values for right figure of figure 34 . 


\begin{tabular}{|c|c|c|c|c|c|c|}
\hline \multirow[b]{2}{*}{$Y$} & \multicolumn{3}{|c|}{$\overline{L L}$} & \multicolumn{3}{|c|}{ NLL } \\
\hline & $\mathrm{MC0}$ & $\mathrm{MC}+$ & MC- & $\mathrm{MC0}$ & MC+ & MC- \\
\hline 6 & 0.0256 & 0.0003 & -0.0003 & 0.0201 & 0.0001 & $\begin{array}{l}-0.0001 \\
\end{array}$ \\
\hline 6.5 & 0.0243 & 0.0001 & -0.0001 & 0.0217 & 0.00008 & -0.00008 \\
\hline 7 & 0.0166 & 0.00007 & -0.00007 & 0.0164 & 0.00005 & -0.00005 \\
\hline 7.5 & 0.00907 & 0.00003 & -0.00003 & 0.00972 & 0.00002 & -0.00002 \\
\hline 8 & 0.00375 & 0.00001 & -0.00001 & 0.00427 & 0.00001 & -0.00001 \\
\hline 8.5 & 0.00121 & 0.000004 & -0.000004 & 0.00144 & 0.000004 & -0.000004 \\
\hline 9 & 0.000314 & 0.000001 & -0.000001 & 0.000389 & 0.000001 & -0.000001 \\
\hline 9.5 & 0.0000564 & 0.0000003 & -0.0000003 & 0.0000707 & 0.0000003 & -0.0000003 \\
\hline 10 & 0.00000496 & 0.00000004 & - 0.00000004 & 0.00000612 & 0.00000005 & -0.00000005 \\
\hline \multicolumn{4}{|c|}{$\mathrm{LL}+$} & & & \\
\hline$Y$ & $\mathrm{MC0}$ & $\mathrm{MC}+$ & MC- & & & \\
\hline 6 & 0.0168 & 0.00009 & -0.00009 & & & \\
\hline 6.5 & 0.0171 & 0.00006 & -0.00006 & & & \\
\hline 7 & 0.0123 & 0.00004 & -0.00004 & & & \\
\hline 7.5 & 0.00698 & 0.00002 & -0.00002 & & & \\
\hline 8 & 0.00297 & 0.000008 & -0.000008 & & & \\
\hline 8.5 & 0.000979 & 0.000003 & -0.000003 & & & \\
\hline 9 & 0.000260 & 0.000001 & -0.000001 & & & \\
\hline 9.5 & 0.0000473 & 0.0000002 & -0.0000002 & & & \\
\hline 10 & 0.00000422 & 0.00000003 & -0.00000007 & & & \\
\hline
\end{tabular}

Table 38. Tabled values for figure 35 .

\begin{tabular}{|l|l|l|l|l|l|l|}
\hline & \multicolumn{7}{|c|}{ LL } & \multicolumn{3}{|c|}{ NLL } \\
$Y$ & $\mu=50$ & $\mu=100$ & $\mu=25$ & $\mu=50$ & $\mu=100$ & $\mu=25$ \\
\hline 6 & 0.0256 & 0.001 & -0.0007 & 0.0201 & -0.0003 & 0.00003 \\
6.5 & 0.0243 & 0.0001 & 0.0003 & 0.0217 & -0.0007 & 0.0003 \\
7 & 0.0166 & -0.0005 & 0.0009 & 0.0164 & -0.0008 & 0.0005 \\
7.5 & 0.00907 & -0.0006 & 0.0008 & 0.00972 & -0.0006 & 0.0005 \\
8 & 0.00375 & -0.0004 & 0.0005 & 0.00427 & -0.0003 & 0.0003 \\
8.5 & 0.00121 & -0.0002 & 0.0002 & 0.00144 & -0.0001 & 0.0001 \\
9 & 0.000314 & -0.00005 & 0.00007 & 0.000389 & -0.00004 & 0.00003 \\
9.5 & 0.0000564 & -0.00001 & 0.00002 & 0.0000707 & -0.000009 & 0.000006 \\
10 & 0.00000496 & -0.000001 & 0.000001 & 0.00000612 & -0.0000009 & 0.0000005 \\
\hline & \multicolumn{7}{|c|}{ LL+ } & & & \\
$Y$ & $\mu=50$ & $\mu=100$ & $\mu=25$ & & & \\
\hline 6 & 0.0168 & -0.003 & 0.005 & & & \\
6.5 & 0.0171 & -0.004 & 0.006 & & & \\
7 & 0.0123 & -0.003 & 0.005 & & & \\
7.5 & 0.00698 & -0.002 & 0.003 & & & \\
8 & 0.00297 & -0.0008 & 0.002 & & & \\
8.5 & 0.000979 & -0.0003 & 0.0006 & & & \\
9 & 0.000260 & -0.00008 & 0.0002 & & & \\
9.5 & 0.0000473 & -0.00002 & 0.00003 & & & \\
10 & 0.00000422 & -0.000002 & 0.000003 &
\end{tabular}

Table 39. Tabled values for left figure of figure 36 . 


\begin{tabular}{|l|l|l|l|l|l|l|}
\hline & \multicolumn{5}{|c|}{ LL } & \multicolumn{3}{c|}{ NLL } \\
$Y$ & $\sqrt{s_{0}}=50$ & $\sqrt{s_{0}}=100$ & $\sqrt{s_{0}}=25$ & $\sqrt{s_{0}}=50$ & $\sqrt{s_{0}}=100$ & $\sqrt{s_{0}}=25$ \\
\hline 6 & 0.0256 & 0.01 & -0.007 & 0.0201 & -0.002 & 0.003 \\
6.5 & 0.0243 & 0.01 & -0.006 & 0.0217 & -0.003 & 0.003 \\
7 & 0.0166 & 0.006 & -0.004 & 0.0164 & -0.002 & 0.002 \\
7.5 & 0.00907 & 0.003 & -0.002 & 0.00972 & -0.001 & 0.001 \\
8 & 0.00375 & 0.001 & -0.0007 & 0.00427 & -0.0006 & 0.0002 \\
8.5 & 0.00121 & 0.0003 & -0.0002 & 0.00144 & -0.0002 & 0.0000006 \\
9 & 0.000314 & 0.00007 & -0.00005 & 0.000389 & -0.00005 & -0.00003 \\
9.5 & 0.0000564 & 0.00001 & -0.000009 & 0.0000707 & -0.000008 & -0.00002 \\
10 & 0.00000496 & 0.000001 & -0.0000008 & 0.00000612 & -0.0000007 & -0.000003 \\
\hline \multicolumn{7}{|c|}{ LL+ } \\
$Y$ & $\sqrt{s_{0}}=50$ & $\sqrt{s_{0}}=100$ & $\sqrt{s_{0}}=25$ & & & \\
\hline 6 & 0.0168 & 0.005 & -0.003 & & & \\
6.5 & 0.0171 & 0.005 & -0.003 & & & \\
7 & 0.0123 & 0.003 & -0.002 & & & \\
7.5 & 0.00698 & 0.002 & -0.001 & & & \\
8 & 0.00297 & 0.0006 & -0.0005 & & & \\
8.5 & 0.000979 & 0.0002 & -0.0002 & & & \\
9 & 0.000260 & 0.00005 & -0.00004 & & & \\
9.5 & 0.0000473 & 0.000009 & -0.000007 & & & \\
10 & 0.00000422 & 0.0000008 & -0.0000006 &
\end{tabular}

Table 40. Tabled values for right figure of figure 36 .

\begin{tabular}{|l|l|l|l|l|}
\hline & LL & \multicolumn{3}{|c|}{ NLL } \\
$Y$ & & MC0 & MC + & MC- \\
\hline 6 & 0.379 & 0.133 & 0.002 & -0.002 \\
6.5 & 0.53 & 0.167 & 0.002 & -0.002 \\
7 & 0.526 & 0.147 & 0.001 & -0.001 \\
7.5 & 0.423 & 0.102 & 0.0007 & -0.0007 \\
8 & 0.271 & 0.0547 & 0.0004 & -0.0004 \\
8.5 & 0.128 & 0.022 & 0.0002 & -0.0002 \\
9 & 0.0508 & 0.00724 & 0.0001 & -0.0001 \\
9.5 & 0.0148 & 0.00167 & 0.00004 & -0.00004 \\
10 & 0.00224 & 0.000187 & 0.000009 & 0.000009 \\
\hline & LL+ & \multicolumn{3}{|c|}{ NLL+ } \\
$Y$ & \multicolumn{4}{|l|}{} \\
\hline 6 & 0.214 & 0.116 & 0.002 & -0.002 \\
6.5 & 0.282 & 0.145 & 0.002 & -0.002 \\
7 & 0.264 & 0.127 & 0.001 & -0.001 \\
7.5 & 0.2 & 0.0878 & 0.0008 & -0.0008 \\
8 & 0.12 & 0.0459 & 0.0005 & -0.0005 \\
8.5 & 0.0529 & 0.0182 & 0.0003 & -0.0003 \\
9 & 0.0197 & 0.00589 & 0.0001 & -0.0001 \\
9.5 & 0.00537 & 0.00135 & 0.00005 & -0.00005 \\
10 & 0.000756 & 0.000158 & 0.00001 & -0.00001 \\
\hline
\end{tabular}

Table 41. Tabled values for figure 37 . 


\begin{tabular}{|l|l|l|l|l|l|l|}
\hline & \multicolumn{5}{|c|}{ LL } & \multicolumn{3}{c|}{ NLL } \\
$Y$ & $\mu=5 \sqrt{70}$ & $\mu=10 \sqrt{70}$ & $\mu=\frac{5}{2} \sqrt{70}$ & $\mu=5 \sqrt{70}$ & $\mu=10 \sqrt{70}$ & $\mu=\frac{5}{2} \sqrt{70}$ \\
\hline 6 & 0.379 & -0.1 & 0.3 & 0.133 & 0.006 & 0.002 \\
6.5 & 0.53 & -0.2 & 0.4 & 0.167 & 0.009 & -0.009 \\
7 & 0.526 & -0.2 & 0.4 & 0.147 & 0.008 & -0.01 \\
7.5 & 0.423 & -0.2 & 0.4 & 0.102 & 0.007 & -0.01 \\
8 & 0.271 & -0.1 & 0.3 & 0.0547 & 0.005 & -0.01 \\
8.5 & 0.128 & -0.06 & 0.2 & 0.022 & 0.002 & -0.004 \\
9 & 0.0508 & -0.03 & 0.07 & 0.00724 & 0.0008 & -0.001 \\
9.5 & 0.0148 & -0.008 & 0.02 & 0.00167 & 0.0002 & -0.0003 \\
10 & 0.00224 & -0.001 & 0.004 & 0.000187 & 0.00002 & -0.000003 \\
\hline & \multicolumn{1}{|c|}{ LL+ } & & \multicolumn{4}{|c|}{ NLL+ } \\
$Y$ & $\mu=5 \sqrt{70}$ & $\mu=10 \sqrt{70}$ & $\mu=\frac{5}{2} \sqrt{70}$ & $\mu=5 \sqrt{70}$ & $\mu=10 \sqrt{70}$ & $\mu=\frac{5}{2} \sqrt{70}$ \\
\hline 6 & 0.214 & -0.06 & 0.1 & 0.116 & 0.003 & 0.003 \\
6.5 & 0.282 & -0.08 & 0.1 & 0.145 & 0.005 & -0.008 \\
7 & 0.264 & -0.08 & 0.1 & 0.127 & 0.006 & -0.01 \\
7.5 & 0.2 & -0.07 & 0.1 & 0.0878 & 0.005 & -0.01 \\
8 & 0.12 & -0.04 & 0.08 & 0.0459 & 0.004 & -0.01 \\
8.5 & 0.0529 & -0.02 & 0.04 & 0.0182 & 0.002 & -0.003 \\
9 & 0.0197 & -0.008 & 0.02 & 0.00589 & 0.0008 & -0.0004 \\
9.5 & 0.00537 & -0.002 & 0.005 & 0.00135 & 0.0002 & 0.0003 \\
10 & 0.000756 & -0.0003 & 0.0007 & 0.000158 & 0.00001 & 0.0002 \\
\hline
\end{tabular}

Table 42. Tabled values for left figure of figure 38 .

\begin{tabular}{|l|l|l|l|l|l|l|}
\hline & \multicolumn{2}{|c|}{ LL } & $\sqrt{c \mid}$ NLL \\
$Y$ & $\sqrt{s_{0}}=5 \sqrt{70}$ & $\sqrt{s_{0}}=10 \sqrt{70}$ & $\sqrt{s_{0}}=\frac{5}{2} \sqrt{70}$ & $\sqrt{s_{0}}=5 \sqrt{70}$ & $\sqrt{s_{0}}=10 \sqrt{70}$ & $\sqrt{s_{0}}=\frac{5}{2} \sqrt{70}$ \\
\hline 6 & 0.379 & -0.06 & 0.07 & 0.133 & 0.02 & -0.01 \\
6.5 & 0.53 & -0.08 & 0.1 & 0.167 & 0.02 & -0.02 \\
7 & 0.526 & -0.08 & 0.1 & 0.147 & 0.02 & -0.01 \\
7.5 & 0.423 & -0.07 & 0.08 & 0.102 & 0.01 & -0.009 \\
8 & 0.271 & -0.04 & 0.05 & 0.0547 & 0.008 & -0.005 \\
8.5 & 0.128 & -0.02 & 0.03 & 0.022 & 0.003 & -0.002 \\
9 & 0.0508 & -0.009 & 0.01 & 0.00724 & 0.001 & -0.0005 \\
9.5 & 0.0148 & -0.003 & 0.003 & 0.00167 & 0.0002 & -0.00006 \\
10 & 0.00224 & -0.0004 & 0.0005 & 0.000187 & 0.00002 & 0.000009 \\
\hline & \multicolumn{2}{|c|}{ LL+ } & & \multicolumn{3}{|c|}{ NLL+ } \\
$Y$ & $\sqrt{s_{0}}=5 \sqrt{70}$ & $\sqrt{s_{0}}=10 \sqrt{70}$ & $\sqrt{s_{0}}=\frac{5}{2} \sqrt{70}$ & $\sqrt{s_{0}}=5 \sqrt{70}$ & $\sqrt{s_{0}}=10 \sqrt{70}$ & $\sqrt{s_{0}}=\frac{5}{2} \sqrt{70}$ \\
\hline 6 & 0.214 & -0.02 & 0.02 & 0.116 & 0.02 & -0.01 \\
6.5 & 0.282 & -0.02 & 0.03 & 0.145 & 0.03 & -0.02 \\
7 & 0.264 & -0.02 & 0.02 & 0.127 & 0.02 & -0.02 \\
7.5 & 0.2 & -0.02 & 0.02 & 0.0878 & 0.02 & -0.01 \\
8 & 0.12 & -0.01 & 0.01 & 0.0459 & 0.01 & -0.005 \\
8.5 & 0.0529 & -0.005 & 0.005 & 0.0182 & 0.004 & -0.002 \\
9 & 0.0197 & -0.002 & 0.002 & 0.00589 & 0.001 & -0.0003 \\
9.5 & 0.00537 & -0.0005 & 0.0006 & 0.00135 & 0.0002 & 0.00007 \\
10 & 0.000756 & -0.00007 & 0.00008 & 0.000158 & 0.000007 & 0.00005 \\
\hline
\end{tabular}

Table 43. Tabled values for right figure of figure 38 . 


\begin{tabular}{|l|l|l|l|l|l|l|}
\hline & \multicolumn{3}{|c|}{ LL } & \multicolumn{3}{|c|}{ NLL } \\
$Y$ & MC0 & MC + & MC- & MC0 & MC+ & MC- \\
\hline 6 & 0.245 & 0.003 & -0.003 & 0.854 & 0.01 & -0.01 \\
6.5 & 0.214 & 0.001 & -0.001 & 0.83 & 0.01 & -0.01 \\
7 & 0.186 & 0.0009 & -0.0009 & 0.811 & 0.008 & -0.008 \\
7.5 & 0.161 & 0.0007 & -0.0007 & 0.802 & 0.007 & -0.007 \\
8 & 0.139 & 0.0005 & -0.0005 & 0.808 & 0.007 & -0.007 \\
8.5 & 0.119 & 0.0005 & -0.0005 & 0.802 & 0.009 & -0.009 \\
9 & 0.102 & 0.0005 & -0.0005 & 0.806 & 0.01 & -0.01 \\
9.5 & 0.0866 & 0.0005 & -0.0005 & 0.824 & 0.02 & -0.02 \\
10 & 0.0736 & 0.0006 & -0.0006 & 0.849 & 0.04 & -0.04 \\
\hline & \multicolumn{7}{|c|}{ LL+ +} & \multicolumn{4}{|c|}{ NLL+ } \\
$Y$ & MC0 & MC+ + & MC- & MC0 & MC+ & MC- \\
\hline 6 & 0.485 & 0.004 & -0.004 & 0.974 & 0.02 & -0.02 \\
6.5 & 0.448 & 0.003 & -0.003 & 0.952 & 0.01 & -0.01 \\
7 & 0.413 & 0.002 & -0.002 & 0.937 & 0.01 & -0.01 \\
7.5 & 0.38 & 0.001 & -0.001 & 0.937 & 0.01 & -0.01 \\
8 & 0.349 & 0.001 & -0.001 & 0.962 & 0.01 & -0.01 \\
8.5 & 0.319 & 0.001 & -0.001 & 0.969 & 0.01 & -0.01 \\
9 & 0.292 & 0.001 & -0.001 & 0.992 & 0.02 & -0.02 \\
9.5 & 0.266 & 0.001 & -0.001 & 1.02 & 0.04 & -0.04 \\
10 & 0.243 & 0.002 & -0.002 & 1.01 & 0.08 & -0.08 \\
\hline
\end{tabular}

Table 44. Tabled values for figure 39.

\begin{tabular}{|l|l|l|l|l|l|l|}
\hline & \multicolumn{5}{|c|}{ LL } & \multicolumn{3}{c|}{ NLL } \\
$Y$ & $\mu=5 \sqrt{70}$ & $\mu=10 \sqrt{70}$ & $\mu=\frac{5}{2} \sqrt{70}$ & $\mu=5 \sqrt{70}$ & $\mu=10 \sqrt{70}$ & $\mu=\frac{5}{2} \sqrt{70}$ \\
\hline 6 & 0.245 & 0.04 & -0.05 & 0.854 & -0.1 & 0.1 \\
6.5 & 0.214 & 0.04 & -0.05 & 0.83 & -0.1 & 0.1 \\
7 & 0.186 & 0.04 & -0.05 & 0.811 & -0.1 & 0.1 \\
7.5 & 0.161 & 0.04 & -0.04 & 0.802 & -0.1 & 0.2 \\
8 & 0.139 & 0.04 & -0.04 & 0.808 & -0.1 & 0.2 \\
8.5 & 0.119 & 0.04 & -0.04 & 0.802 & -0.1 & 0.2 \\
9 & 0.102 & 0.04 & -0.03 & 0.806 & -0.2 & 0.2 \\
9.5 & 0.0866 & 0.03 & -0.03 & 0.824 & -0.2 & 0.1 \\
10 & 0.0736 & 0.03 & -0.03 & 0.849 & -0.2 & -0.08 \\
\hline & \multicolumn{2}{|c|}{ LL+ } & & \multicolumn{3}{|c|}{ NLL+ } \\
$Y$ & $\mu=5 \sqrt{70}$ & $\mu=10 \sqrt{70}$ & $\mu=\frac{5}{2} \sqrt{70}$ & $\mu=5 \sqrt{70}$ & $\mu=10 \sqrt{70}$ & $\mu=\frac{5}{2} \sqrt{70}$ \\
\hline 6 & 0.485 & 0.01 & -0.01 & 0.974 & -0.09 & 0.1 \\
6.5 & 0.448 & 0.01 & -0.01 & 0.952 & -0.1 & 0.1 \\
7 & 0.413 & 0.01 & -0.01 & 0.937 & -0.1 & 0.2 \\
7.5 & 0.38 & 0.01 & -0.01 & 0.937 & -0.1 & 0.2 \\
8 & 0.349 & 0.01 & -0.01 & 0.962 & -0.2 & 0.3 \\
8.5 & 0.319 & 0.01 & -0.01 & 0.969 & -0.2 & 0.2 \\
9 & 0.292 & 0.01 & -0.01 & 0.992 & -0.2 & 0.06 \\
9.5 & 0.266 & 0.01 & -0.01 & 1.02 & -0.2 & -0.2 \\
10 & 0.243 & 0.01 & -0.01 & 1.01 & -0.1 & -0.6 \\
\hline
\end{tabular}

Table 45. Tabled values for figure 40 . 


\begin{tabular}{|l|l|l|l|l|l|l|}
\hline & \multicolumn{2}{|c|}{ LL } & $\sqrt{c \mid}$ NLL & \\
$Y$ & $\sqrt{s_{0}}=5 \sqrt{70}$ & $\sqrt{s_{0}}=10 \sqrt{70}$ & $\sqrt{s_{0}}=17.5$ & $\sqrt{s_{0}}=5 \sqrt{70}$ & $\sqrt{s_{0}}=10 \sqrt{70}$ & $\sqrt{s_{0}}=\frac{5}{2} \sqrt{70}$ \\
\hline 6 & 0.245 & 0.05 & -0.04 & 0.854 & -0.1 & 0.1 \\
6.5 & 0.214 & 0.04 & -0.04 & 0.83 & -0.1 & 0.1 \\
7 & 0.186 & 0.04 & -0.03 & 0.811 & -0.1 & 0.1 \\
7.5 & 0.161 & 0.04 & -0.03 & 0.802 & -0.1 & 0.1 \\
8 & 0.139 & 0.03 & -0.03 & 0.808 & -0.1 & 0.1 \\
8.5 & 0.119 & 0.03 & -0.02 & 0.802 & -0.1 & 0.1 \\
9 & 0.102 & 0.02 & -0.02 & 0.806 & -0.1 & 0.1 \\
9.5 & 0.0866 & 0.02 & -0.02 & 0.824 & -0.1 & 0.07 \\
10 & 0.0736 & 0.02 & -0.02 & 0.849 & -0.1 & 0.004 \\
\hline & & \multicolumn{2}{|c|}{ LL+ } & & \multicolumn{3}{c}{ NLL+ } \\
$Y$ & $\sqrt{s_{0}}=5 \sqrt{70}$ & $\sqrt{s_{0}}=10 \sqrt{70}$ & $\sqrt{s_{0}}=\frac{5}{2} \sqrt{70}$ & $\sqrt{s_{0}}=5 \sqrt{70}$ & $\sqrt{s_{0}}=10 \sqrt{70}$ & $\sqrt{s_{0}}=\frac{5}{2} \sqrt{70}$ \\
\hline 6 & 0.485 & 0.05 & -0.05 & 0.974 & -0.2 & 0.2 \\
6.5 & 0.448 & 0.05 & -0.05 & 0.952 & -0.2 & 0.2 \\
7 & 0.413 & 0.05 & -0.05 & 0.937 & -0.2 & 0.2 \\
7.5 & 0.38 & 0.05 & -0.04 & 0.937 & -0.2 & 0.2 \\
8 & 0.349 & 0.04 & -0.04 & 0.962 & -0.2 & 0.2 \\
8.5 & 0.319 & 0.04 & -0.04 & 0.969 & -0.2 & 0.1 \\
9 & 0.292 & 0.04 & -0.03 & 0.992 & -0.2 & 0.1 \\
9.5 & 0.266 & 0.04 & -0.03 & 1.02 & -0.2 & 0.001 \\
10 & 0.243 & 0.03 & -0.03 & 1.01 & -0.09 & -0.2 \\
\hline
\end{tabular}

Table 46. Tabled values for figure 41 .

\begin{tabular}{|l|l|c|c|c|c|c|}
\hline & \multicolumn{3}{|c|}{ LL } & \multicolumn{3}{c|}{ NLL } \\
$Y$ & MC0 & MC+ & MC- & MC0 & MC+ & MC- \\
\hline 6 & 0.111 & 0.002 & -0.002 & 0.437 & 0.008 & -0.008 \\
6.5 & 0.0921 & 0.0009 & -0.0009 & 0.407 & 0.005 & -0.005 \\
7 & 0.076 & 0.0006 & -0.0006 & 0.381 & 0.004 & -0.004 \\
7.5 & 0.0623 & 0.0004 & -0.0004 & 0.363 & 0.003 & -0.003 \\
8 & 0.0508 & 0.0003 & -0.0003 & 0.354 & 0.003 & -0.003 \\
8.5 & 0.0411 & 0.0002 & -0.0002 & 0.339 & 0.004 & -0.004 \\
9 & 0.0332 & 0.0002 & -0.0002 & 0.331 & 0.005 & -0.005 \\
9.5 & 0.0266 & 0.0002 & -0.0002 & 0.333 & 0.008 & -0.008 \\
10 & 0.0213 & 0.0002 & -0.0002 & 0.347 & 0.02 & -0.02 \\
\hline & \multicolumn{7}{|c|}{ LL+ +} & \multicolumn{4}{|c|}{ NLL+ } \\
$Y$ & MC0 & MC+ & MC- & MC0 & MC+ & MC- \\
\hline 6 & 0.209 & 0.003 & -0.003 & 0.498 & 0.01 & -0.01 \\
6.5 & 0.182 & 0.001 & -0.001 & 0.467 & 0.006 & -0.006 \\
7 & 0.158 & 0.0009 & -0.0009 & 0.44 & 0.005 & -0.005 \\
7.5 & 0.137 & 0.0006 & -0.0006 & 0.424 & 0.004 & -0.004 \\
8 & 0.118 & 0.0005 & -0.0005 & 0.422 & 0.005 & -0.005 \\
8.5 & 0.101 & 0.0005 & -0.0005 & 0.41 & 0.006 & -0.006 \\
9 & 0.0862 & 0.0004 & -0.0004 & 0.408 & 0.009 & -0.009 \\
9.5 & 0.0734 & 0.0005 & -0.0005 & 0.415 & 0.01 & -0.01 \\
10 & 0.0624 & 0.0006 & -0.0006 & 0.412 & 0.03 & -0.03 \\
\hline
\end{tabular}

Table 47. Tabled values for figure 42 . 


\begin{tabular}{|l|l|l|l|l|l|l|}
\hline & \multicolumn{5}{|c|}{ LL } & \multicolumn{3}{c|}{ NLL } \\
$Y$ & $\mu=5 \sqrt{70}$ & $\mu=10 \sqrt{70}$ & $\mu=\frac{5}{2} \sqrt{70}$ & $\mu=5 \sqrt{70}$ & $\mu=10 \sqrt{70}$ & $\mu=\frac{5}{2} \sqrt{70}$ \\
\hline 6 & 0.111 & 0.03 & -0.03 & 0.437 & -0.05 & 0.02 \\
6.5 & 0.0921 & 0.03 & -0.03 & 0.407 & -0.05 & 0.05 \\
7 & 0.076 & 0.02 & -0.02 & 0.381 & -0.05 & 0.07 \\
7.5 & 0.0623 & 0.02 & -0.02 & 0.363 & -0.06 & 0.09 \\
8 & 0.0508 & 0.02 & -0.02 & 0.354 & -0.06 & 0.1 \\
8.5 & 0.0411 & 0.02 & -0.02 & 0.339 & -0.07 & 0.1 \\
9 & 0.0332 & 0.02 & -0.01 & 0.331 & -0.07 & 0.1 \\
9.5 & 0.0266 & 0.01 & -0.01 & 0.333 & -0.08 & 0.1 \\
10 & 0.0213 & 0.01 & -0.01 & 0.347 & -0.08 & 0.04 \\
\hline & \multicolumn{1}{|c|}{ LL+ } & & \multicolumn{3}{|c|}{ NLL+ } \\
$Y$ & $\mu=5 \sqrt{70}$ & $\mu=10 \sqrt{70}$ & $\mu=\frac{5}{2} \sqrt{70}$ & $\mu=5 \sqrt{70}$ & $\mu=10 \sqrt{70}$ & $\mu=\frac{5}{2} \sqrt{70}$ \\
\hline 6 & 0.209 & 0.009 & -0.008 & 0.498 & -0.05 & 0.02 \\
6.5 & 0.182 & 0.009 & -0.007 & 0.467 & -0.05 & 0.06 \\
7 & 0.158 & 0.009 & -0.007 & 0.44 & -0.06 & 0.09 \\
7.5 & 0.137 & 0.009 & -0.007 & 0.424 & -0.07 & 0.1 \\
8 & 0.118 & 0.008 & -0.006 & 0.422 & -0.08 & 0.2 \\
8.5 & 0.101 & 0.008 & -0.006 & 0.41 & -0.09 & 0.1 \\
9 & 0.0862 & 0.007 & -0.005 & 0.408 & -0.1 & 0.09 \\
9.5 & 0.0734 & 0.007 & -0.005 & 0.415 & -0.1 & -0.04 \\
10 & 0.0624 & 0.006 & -0.005 & 0.412 & -0.08 & -0.2 \\
\hline
\end{tabular}

Table 48. Tabled values for figure 43.

\begin{tabular}{|l|l|l|l|l|l|l|}
\hline$Y$ & $\sqrt{s_{0}}=5 \sqrt{70}$ & $\sqrt{s_{0}}=10 \sqrt{70}$ & $\sqrt{s_{0}}=17.5$ & $\sqrt{s_{0}}=5 \sqrt{70}$ & $\sqrt{s_{0}}=10 \sqrt{70}$ & $\sqrt{s_{0}}=\frac{5}{2} \sqrt{70}$ \\
\hline 6 & 0.111 & 0.03 & -0.03 & 0.437 & -0.09 & 0.09 \\
6.5 & 0.0921 & 0.03 & -0.02 & 0.407 & -0.08 & 0.08 \\
7 & 0.076 & 0.02 & -0.02 & 0.381 & -0.08 & 0.08 \\
7.5 & 0.0623 & 0.02 & -0.02 & 0.363 & -0.08 & 0.07 \\
8 & 0.0508 & 0.02 & -0.01 & 0.354 & -0.08 & 0.06 \\
8.5 & 0.0411 & 0.01 & -0.01 & 0.339 & -0.07 & 0.05 \\
9 & 0.0332 & 0.01 & -0.009 & 0.331 & -0.07 & 0.03 \\
9.5 & 0.0266 & 0.009 & -0.007 & 0.333 & -0.07 & -0.001 \\
10 & 0.0213 & 0.008 & -0.006 & 0.347 & -0.06 & -0.1 \\
\hline & \multicolumn{2}{|c|}{ LL+ } & & \multicolumn{3}{|c|}{ NLL+ } \\
$Y$ & $\sqrt{s_{0}}=5 \sqrt{70}$ & $\sqrt{s_{0}}=10 \sqrt{70}$ & $\sqrt{s_{0}}=\frac{5}{2} \sqrt{70}$ & $\sqrt{s_{0}}=5 \sqrt{70}$ & $\sqrt{s_{0}}=10 \sqrt{70}$ & $\sqrt{s_{0}}=\frac{5}{2} \sqrt{70}$ \\
\hline 6 & 0.209 & 0.04 & -0.04 & 0.498 & -0.1 & 0.1 \\
6.5 & 0.182 & 0.04 & -0.03 & 0.467 & -0.1 & 0.1 \\
7 & 0.158 & 0.03 & -0.03 & 0.44 & -0.1 & 0.1 \\
7.5 & 0.137 & 0.03 & -0.03 & 0.424 & -0.1 & 0.1 \\
8 & 0.118 & 0.03 & -0.02 & 0.422 & -0.1 & 0.08 \\
8.5 & 0.101 & 0.02 & -0.02 & 0.41 & -0.1 & 0.06 \\
9 & 0.0862 & 0.02 & -0.02 & 0.408 & -0.1 & 0.03 \\
9.5 & 0.0734 & 0.02 & -0.01 & 0.415 & -0.1 & -0.04 \\
10 & 0.0624 & 0.02 & -0.01 & 0.412 & -0.06 & -0.2 \\
\hline
\end{tabular}

Table 49. Tabled values for figure 44 . 


\begin{tabular}{|l|l|c|c|c|c|c|}
\hline & \multicolumn{3}{|c|}{ LL } & \multicolumn{3}{c|}{ NLL } \\
$Y$ & MC0 & MC+ & MC- & MC0 & MC+ & MC- \\
\hline 6 & 0.452 & 0.009 & -0.009 & 0.511 & 0.009 & -0.009 \\
6.5 & 0.43 & 0.005 & -0.005 & 0.49 & 0.005 & -0.005 \\
7 & 0.408 & 0.004 & -0.004 & 0.47 & 0.004 & -0.004 \\
7.5 & 0.387 & 0.003 & -0.003 & 0.453 & 0.003 & -0.003 \\
8 & 0.366 & 0.002 & -0.002 & 0.438 & 0.003 & -0.003 \\
8.5 & 0.346 & 0.002 & -0.002 & 0.423 & 0.003 & -0.003 \\
9 & 0.326 & 0.002 & -0.002 & 0.411 & 0.003 & -0.003 \\
9.5 & 0.307 & 0.003 & -0.003 & 0.405 & 0.004 & -0.004 \\
10 & 0.289 & 0.004 & -0.004 & 0.408 & 0.007 & -0.007 \\
\hline & \multicolumn{7}{|c|}{ LL+ +} & & & \\
$Y$ & MC0 & MC+ + & MC- & & & \\
\hline 6 & 0.43 & 0.006 & -0.006 & & & \\
6.5 & 0.406 & 0.004 & -0.004 & & & \\
7 & 0.382 & 0.003 & -0.003 & & & \\
7.5 & 0.359 & 0.002 & -0.002 & & & \\
8 & 0.337 & 0.002 & -0.002 & &
\end{tabular}

Table 50. Tabled values for figure 45 .

\begin{tabular}{|c|c|c|c|c|c|c|}
\hline & \multicolumn{3}{|c|}{ LL } & \multicolumn{3}{|c|}{ NLL } \\
\hline$Y$ & $\mu=5 \sqrt{70}$ & $\mu=10 \sqrt{70}$ & $\mu=\frac{5}{2} \sqrt{70}$ & $\mu=5 \sqrt{70}$ & $\mu=10 \sqrt{70}$ & $\mu=\frac{5}{2} \sqrt{70}$ \\
\hline 6 & 0.452 & 0.03 & -0.04 & 0.511 & -0.001 & -0.03 \\
\hline 6.5 & 0.43 & 0.03 & -0.04 & 0.49 & -0.003 & -0.008 \\
\hline 7 & 0.408 & 0.03 & -0.04 & 0.47 & -0.004 & 0.006 \\
\hline 7.5 & 0.387 & 0.03 & -0.04 & 0.453 & -0.006 & 0.01 \\
\hline 8 & 0.366 & 0.04 & -0.04 & 0.438 & -0.009 & 0.03 \\
\hline 8.5 & 0.346 & 0.04 & -0.04 & 0.423 & -0.01 & 0.04 \\
\hline 9 & 0.326 & 0.04 & -0.05 & 0.411 & -0.01 & 0.05 \\
\hline 9.5 & 0.307 & 0.04 & -0.05 & 0.405 & -0.02 & 0.06 \\
\hline 10 & 0.289 & 0.04 & -0.05 & 0.408 & -0.03 & 0.09 \\
\hline$Y$ & & $\begin{array}{c}\mathrm{LL}+ \\
\mathrm{c}\end{array}$ & $5 \sqrt{70}$ & & & \\
\hline$\frac{Y}{6}$ & $\mu=5 \sqrt{ } 70$ & $\mu=10 \sqrt{70}$ & $\mu=\frac{\overline{2}}{2} \sqrt{ } 70$ & & & \\
\hline 6 & 0.43 & 0.009 & -0.005 & & & \\
\hline 6.5 & 0.406 & 0.01 & -0.005 & & & \\
\hline 7 & 0.382 & 0.01 & -0.006 & & & \\
\hline 7.5 & 0.359 & 0.01 & -0.006 & & & \\
\hline 8 & 0.337 & 0.01 & -0.006 & & & \\
\hline 8.5 & 0.316 & 0.01 & -0.006 & & & \\
\hline 9 & 0.295 & 0.01 & -0.006 & & & \\
\hline 9.5 & 0.276 & 0.01 & -0.006 & & & \\
\hline 10 & 0.257 & 0.01 & -0.006 & & & \\
\hline
\end{tabular}

Table 51. Tabled values for figure 46 . 


\begin{tabular}{|c|c|c|c|c|c|c|}
\hline & \multicolumn{3}{|c|}{ LL } & \multicolumn{3}{|c|}{ NLL } \\
\hline$Y$ & $\sqrt{s_{0}}=5 \sqrt{70}$ & $\sqrt{s_{0}}=10 \sqrt{70}$ & $\sqrt{s_{0}}=17.5$ & $\sqrt{s_{0}}=5 \sqrt{70}$ & $\sqrt{s_{0}}=10 \sqrt{70}$ & $\sqrt{s_{0}}=\frac{5}{2} \sqrt{70}$ \\
\hline 6 & 0.452 & 0.03 & -0.03 & 0.511 & -0.03 & 0.02 \\
\hline 6.5 & 0.43 & 0.03 & -0.03 & 0.49 & -0.03 & 0.02 \\
\hline 7 & 0.408 & 0.03 & -0.03 & 0.47 & -0.03 & 0.02 \\
\hline 7.5 & 0.387 & 0.03 & -0.03 & 0.453 & -0.02 & 0.02 \\
\hline 8 & 0.366 & 0.03 & -0.03 & 0.438 & -0.02 & 0.007 \\
\hline 8.5 & 0.346 & 0.03 & -0.03 & 0.423 & -0.02 & 0.001 \\
\hline 9 & 0.326 & 0.03 & -0.03 & 0.411 & -0.02 & -0.009 \\
\hline 9.5 & 0.307 & 0.03 & -0.03 & 0.405 & -0.02 & -0.04 \\
\hline 10 & 0.289 & 0.03 & -0.02 & 0.408 & -0.02 & -0.1 \\
\hline$Y$ & $\sqrt{s_{0}}=5 \sqrt{70}$ & $\begin{array}{c}\text { LL+ } \\
\sqrt{s_{0}}=10 \sqrt{70}\end{array}$ & $\sqrt{s_{0}}=\frac{5}{2} \sqrt{70}$ & & & \\
\hline 6 & 0.43 & 0.03 & -0.03 & & & \\
\hline 6.5 & 0.406 & 0.03 & -0.03 & & & \\
\hline 7 & 0.382 & 0.03 & -0.03 & & & \\
\hline 7.5 & 0.359 & 0.03 & -0.03 & & & \\
\hline 8 & 0.337 & 0.03 & -0.03 & & & \\
\hline 8.5 & 0.316 & 0.03 & -0.03 & & & \\
\hline 9 & 0.295 & 0.03 & -0.03 & & & \\
\hline 9.5 & 0.276 & 0.03 & -0.03 & & & \\
\hline 10 & 0.257 & 0.03 & -0.02 & & & \\
\hline
\end{tabular}

Table 52. Tabled values for figure 47 .

\begin{tabular}{|l|l|l|l|l|l|l|}
\hline & \multicolumn{7}{|c|}{ LL } & \multicolumn{3}{c|}{ NLL } \\
$Y$ & MC0 & MC+ & MC- & MC0 & MC + & MC- \\
\hline 6 & 0.0464 & 0.0005 & -0.0005 & 0.0566 & 0.0006 & -0.0006 \\
6.5 & 0.0567 & 0.0004 & -0.0004 & 0.0692 & 0.0005 & -0.0005 \\
7 & 0.0489 & 0.0002 & -0.0002 & 0.0596 & 0.0003 & -0.0003 \\
7.5 & 0.0341 & 0.0001 & -0.0001 & 0.0411 & 0.0002 & -0.0002 \\
8 & 0.0188 & 0.00007 & -0.00007 & 0.0221 & 0.0001 & -0.0001 \\
8.5 & 0.00761 & 0.00003 & -0.00003 & 0.00884 & 0.00004 & -0.00004 \\
9 & 0.00258 & 0.00001 & -0.00001 & 0.00292 & 0.00002 & -0.00002 \\
9.5 & 0.000642 & 0.000004 & -0.000004 & 0.00069 & 0.000006 & -0.000006 \\
10 & 0.0000824 & 0.0000007 & -0.0000007 & 0.0000796 & 0.000001 & -0.000001 \\
\hline & \multicolumn{7}{|c|}{ LL+ +} & & & \\
$Y$ & MC0 & MC+ + & MC- & & & \\
\hline 6 & 0.0519 & 0.0005 & -0.0005 & & & \\
6.5 & 0.0633 & 0.0004 & -0.0004 & & & \\
7 & 0.0545 & 0.0002 & -0.0002 & & & \\
7.5 & 0.0379 & 0.0001 & -0.0001 & & & \\
8 & 0.0209 & 0.00007 & -0.00007 & & & \\
8.5 & 0.00846 & 0.00003 & -0.00003 & & & \\
9 & 0.00287 & 0.00001 & -0.00001 & & & \\
9.5 & 0.000715 & 0.000004 & -0.000004 & & \\
10 & 0.0000918 & 0.0000007 & -0.0000007 &
\end{tabular}

Table 53. Tabled values for figure 48 . 


\begin{tabular}{|c|c|c|c|c|c|c|}
\hline & \multicolumn{3}{|c|}{ LL } & \multicolumn{3}{|c|}{ NLL } \\
\hline Y & $\mu=5 \sqrt{70}$ & $\mu=10 \sqrt{70}$ & $\mu=\frac{5}{2} \sqrt{70}$ & $\mu=5 \sqrt{70}$ & $\mu=10 \sqrt{70}$ & $\mu=\frac{5}{2} \sqrt{70}$ \\
\hline 6 & 0.0464 & -0.01 & 0.02 & 0.0566 & -0.004 & 0.008 \\
\hline 6.5 & 0.0567 & -0.01 & 0.02 & 0.0692 & -0.005 & 0.006 \\
\hline 7 & 0.0489 & -0.01 & 0.02 & 0.0596 & -0.005 & 0.004 \\
\hline 7.5 & 0.0341 & -0.01 & 0.01 & 0.0411 & -0.004 & 0.002 \\
\hline 8 & 0.0188 & -0.006 & 0.009 & 0.0221 & -0.002 & 0.0005 \\
\hline 8.5 & 0.00761 & -0.002 & 0.004 & 0.00884 & -0.0009 & -0.000006 \\
\hline 9 & 0.00258 & -0.0008 & 0.001 & 0.00292 & -0.0003 & -0.00005 \\
\hline 9.5 & 0.000642 & -0.0002 & 0.0004 & 0.00069 & -0.00007 & -0.00003 \\
\hline 10 & 0.0000824 & -0.00003 & 0.00006 & 0.0000796 & -0.000007 & -0.000008 \\
\hline \multicolumn{4}{|c|}{ LL+ } & & & \\
\hline$\frac{Y}{6}$ & $\frac{\mu=5 \sqrt{ } 70}{0.0519}$ & $\frac{\mu=10 \sqrt{ } \mathrm{r} 0}{-0.01}$ & $\frac{\mu=\frac{1}{2} \sqrt{ } 70}{0.02}$ & & & \\
\hline 6.5 & 0.0633 & -0.02 & 0.03 & & & \\
\hline 7 & 0.0545 & -0.02 & 0.03 & & & \\
\hline 7.5 & 0.0379 & -0.01 & 0.02 & & & \\
\hline 8 & 0.0209 & -0.007 & 0.01 & & & \\
\hline 8.5 & 0.00846 & -0.003 & 0.005 & & & \\
\hline 9 & 0.00287 & -0.001 & 0.002 & & & \\
\hline 9.5 & 0.000715 & -0.0003 & 0.0006 & & & \\
\hline 10 & 0.0000918 & -0.00004 & 0.00008 & & & \\
\hline
\end{tabular}

Table 54. Tabled values for left figure of figure 49 .

\begin{tabular}{|c|c|c|c|c|c|c|}
\hline & \multicolumn{3}{|c|}{ LL } & \multicolumn{3}{|c|}{ NLL } \\
\hline$Y$ & $\sqrt{s_{0}}=5 \sqrt{70}$ & $\sqrt{s_{0}}=10 \sqrt{70}$ & $\sqrt{s_{0}}=\frac{5}{2} \sqrt{70}$ & $\sqrt{s_{0}}=5 \sqrt{70}$ & $\sqrt{s_{0}}=10 \sqrt{70}$ & $\sqrt{s_{0}}=\frac{5}{2} \sqrt{70}$ \\
\hline 6 & 0.0464 & 0.0004 & -0.0008 & 0.0566 & -0.002 & 0.003 \\
\hline 6.5 & 0.0567 & 0.0009 & -0.001 & 0.0692 & -0.003 & 0.003 \\
\hline 7 & 0.0489 & 0.001 & -0.001 & 0.0596 & -0.003 & 0.003 \\
\hline 7.5 & 0.0341 & 0.0008 & -0.0009 & 0.0411 & -0.002 & 0.002 \\
\hline 8 & 0.0188 & 0.0005 & -0.0005 & 0.0221 & -0.001 & 0.001 \\
\hline 8.5 & 0.00761 & 0.0002 & -0.0002 & 0.00884 & -0.0004 & 0.0004 \\
\hline 9 & 0.00258 & 0.00008 & -0.00008 & 0.00292 & -0.0001 & 0.0001 \\
\hline 9.5 & 0.000642 & 0.00002 & -0.00002 & 0.00069 & -0.00004 & 0.00003 \\
\hline 10 & 0.0000824 & 0.000003 & -0.000003 & 0.0000796 & -0.000004 & 0.000004 \\
\hline$Y$ & $\sqrt{s_{0}}=5 \sqrt{70}$ & $\begin{array}{c}\text { LL+ } \\
\sqrt{s_{0}}=10 \sqrt{70}\end{array}$ & $\sqrt{s_{0}}=\frac{5}{2} \sqrt{70}$ & & & \\
\hline 6 & 0.0519 & 0.0007 & -0.001 & & & \\
\hline 6.5 & 0.0633 & 0.001 & -0.002 & & & \\
\hline 7 & 0.0545 & 0.001 & -0.001 & & & \\
\hline 7.5 & 0.0379 & 0.001 & -0.001 & & & \\
\hline 8 & 0.0209 & 0.0006 & -0.0006 & & & \\
\hline 8.5 & 0.00846 & 0.0002 & -0.0002 & & & \\
\hline 9 & 0.00287 & 0.00009 & -0.00008 & & & \\
\hline 9.5 & 0.000715 & 0.00002 & -0.00002 & & & \\
\hline 10 & 0.0000918 & 0.000003 & -0.000003 & & & \\
\hline
\end{tabular}

Table 55. Tabled values for right figure of figure 49 . 


\begin{tabular}{|l|l|l|l|l|l|l|}
\hline & \multicolumn{7}{|c|}{ LL } & \multicolumn{3}{c|}{ NLL } \\
$Y$ & MC0 & MC+ & MC- & MC0 & MC + & MC- \\
\hline 6 & 0.021 & 0.0004 & -0.0004 & 0.029 & 0.0004 & -0.0004 \\
6.5 & 0.0244 & 0.0003 & -0.0003 & 0.0339 & 0.0003 & -0.0003 \\
7 & 0.02 & 0.0001 & -0.0001 & 0.028 & 0.0002 & -0.0002 \\
7.5 & 0.0132 & 0.00008 & -0.00008 & 0.0186 & 0.00008 & -0.00008 \\
8 & 0.00687 & 0.00004 & -0.00004 & 0.00968 & 0.00004 & -0.00004 \\
8.5 & 0.00263 & 0.00001 & -0.00001 & 0.00374 & 0.00002 & -0.00002 \\
9 & 0.000841 & 0.000005 & -0.000005 & 0.0012 & 0.000006 & -0.000006 \\
9.5 & 0.000197 & 0.000002 & -0.000001 & 0.000279 & 0.000002 & -0.000002 \\
10 & 0.0000238 & 0.0000003 & -0.0000003 & 0.0000325 & 0.0000003 & -0.0000003 \\
\hline & \multicolumn{7}{|c|}{ LL+ +} & & & \\
$Y$ & $\mathrm{MC} 0$ & $\mathrm{MC}+$ & $\mathrm{MC}-$ & & & \\
\hline 6 & 0.0223 & 0.0003 & -0.0003 & & & \\
6.5 & 0.0257 & 0.0002 & -0.0002 & & & \\
7 & 0.0208 & 0.0001 & -0.0001 & & & \\
7.5 & 0.0136 & 0.00006 & -0.00006 & & & \\
8 & 0.00703 & 0.00003 & -0.00003 & & & \\
8.5 & 0.00267 & 0.00001 & -0.00001 & & & \\
9 & 0.000847 & 0.000004 & -0.000004 & & & \\
9.5 & 0.000197 & 0.000001 & -0.000001 & & & \\
10 & 0.0000236 & 0.0000002 & -0.0000002 & &
\end{tabular}

Table 56. Tabled values for figure 50 .

\begin{tabular}{|c|c|c|c|c|c|c|}
\hline & \multicolumn{3}{|c|}{$\mathrm{LL}$} & \multicolumn{3}{|c|}{ NLL } \\
\hline$Y$ & $\mu=5 \sqrt{70}$ & $\mu=10 \sqrt{70}$ & $\mu=\frac{5}{2} \sqrt{70}$ & $\mu=5 \sqrt{70}$ & $\mu=10 \sqrt{70}$ & $\mu=\frac{5}{2} \sqrt{70}$ \\
\hline 6 & 0.021 & -0.004 & 0.005 & 0.029 & -0.002 & 0.002 \\
\hline 6.5 & 0.0244 & -0.005 & 0.006 & 0.0339 & -0.003 & 0.002 \\
\hline 7 & 0.02 & -0.004 & 0.005 & 0.028 & -0.003 & 0.002 \\
\hline 7.5 & 0.0132 & -0.003 & 0.004 & 0.0186 & -0.002 & 0.002 \\
\hline 8 & 0.00687 & -0.002 & 0.002 & 0.00968 & -0.001 & 0.0009 \\
\hline 8.5 & 0.00263 & -0.0006 & 0.0008 & 0.00374 & -0.0005 & 0.0004 \\
\hline 9 & 0.000841 & -0.0002 & 0.0003 & 0.0012 & -0.0002 & 0.0001 \\
\hline 9.5 & 0.000197 & -0.00005 & 0.00007 & 0.000279 & -0.00004 & 0.00003 \\
\hline 10 & 0.0000238 & -0.000007 & 0.00001 & 0.0000325 & -0.000005 & 0.000003 \\
\hline Y & $\mu=5 \sqrt{70}$ & $\begin{array}{c}\text { LL+ } \\
\mu=10 \sqrt{70}\end{array}$ & $\mu=\frac{5}{2} \sqrt{70}$ & & & \\
\hline 6 & 0.0223 & -0.006 & 0.009 & & & \\
\hline 6.5 & 0.0257 & -0.007 & 0.01 & & & \\
\hline 7 & 0.0208 & -0.006 & 0.01 & & & \\
\hline 7.5 & 0.0136 & -0.004 & 0.007 & & & \\
\hline 8 & 0.00703 & -0.002 & 0.004 & & & \\
\hline 8.5 & 0.00267 & -0.0009 & 0.002 & & & \\
\hline 9 & 0.000847 & -0.0003 & 0.0006 & & & \\
\hline 9.5 & 0.000197 & -0.00007 & 0.0001 & & & \\
\hline 10 & 0.0000236 & -0.000009 & 0.00002 & & & \\
\hline
\end{tabular}

Table 57. Tabled values for left figure of figure 51. 


\begin{tabular}{|c|c|c|c|c|c|c|}
\hline$Y$ & $\sqrt{s_{0}}=5 \sqrt{70}$ & $\mathrm{LL}$ & $\sqrt{s_{0}}=\frac{5}{2} \sqrt{70}$ & $\sqrt{s_{0}}=5 \sqrt{70}$ & NLL & $\sqrt{s_{0}}=\frac{5}{2} \sqrt{70}$ \\
\hline 6 & 0.021 & 0.002 & -0.002 & 0.029 & -0.003 & 0.003 \\
\hline 6.5 & 0.0244 & 0.002 & -0.002 & 0.0339 & -0.003 & 0.003 \\
\hline 7 & 0.02 & 0.002 & -0.002 & 0.028 & -0.003 & 0.002 \\
\hline 7.5 & 0.0132 & 0.001 & -0.001 & 0.0186 & -0.002 & 0.002 \\
\hline 8 & 0.00687 & 0.0007 & -0.0007 & 0.00968 & -0.001 & 0.0006 \\
\hline 8.5 & 0.00263 & 0.0003 & -0.0003 & 0.00374 & -0.0004 & 0.0002 \\
\hline 9 & 0.000841 & 0.0001 & -0.00009 & 0.0012 & -0.0001 & 0.00003 \\
\hline 9.5 & 0.000197 & 0.00002 & -0.00002 & 0.000279 & -0.00003 & -0.00001 \\
\hline 10 & 0.0000238 & 0.000003 & -0.000003 & 0.0000325 & -0.000003 & -0.000009 \\
\hline$Y$ & $\sqrt{s_{0}}=5 \sqrt{70}$ & $\begin{array}{c}\text { LL+ } \\
\sqrt{s_{0}}=10 \sqrt{70}\end{array}$ & $\sqrt{s_{0}}=\frac{5}{2} \sqrt{70}$ & & & \\
\hline 6 & 0.0223 & 0.002 & -0.002 & & & \\
\hline 6.5 & 0.0257 & 0.003 & -0.003 & & & \\
\hline 7 & 0.0208 & 0.002 & -0.002 & & & \\
\hline 7.5 & 0.0136 & 0.002 & -0.002 & & & \\
\hline 8 & 0.00703 & 0.0009 & -0.0008 & & & \\
\hline 8.5 & 0.00267 & 0.0003 & -0.0003 & & & \\
\hline 9 & 0.000847 & 0.0001 & -0.0001 & & & \\
\hline 9.5 & 0.000197 & 0.00003 & -0.00002 & & & \\
\hline 10 & 0.0000236 & 0.000003 & -0.000003 & & & \\
\hline
\end{tabular}

Table 58. Tabled values for right figure of figure 51.

Open Access. This article is distributed under the terms of the Creative Commons Attribution Noncommercial License which permits any noncommercial use, distribution, and reproduction in any medium, provided the original author(s) and source are credited.

\section{References}

[1] V.S. Fadin, E.A. Kuraev and L.N. Lipatov, On the Pomeranchuk singularity in asymptotically free theories, Phys. Lett. B 60 (1975) 50 [SPIRES].

[2] E.A. Kuraev, L.N. Lipatov and V.S. Fadin, Multi-Reggeon processes in the Yang-Mills theory, Sov. Phys. JETP 44 (1976) 443 [Zh. Eksp. Teor. Fiz. 71 (1976) 840] [SPIRES].

[3] E.A. Kuraev, L.N. Lipatov and V.S. Fadin, The Pomeranchuk singularity in non-Abelian gauge theories, Sov. Phys. JETP 45 (1977) 199 [Zh. Eksp. Teor. Fiz. 72 (1977) 377] [SPIRES].

[4] I.I. Balitsky and L.N. Lipatov, The Pomeranchuk singularity in quantum chromodynamics, Sov. J. Nucl. Phys. 28 (1978) 822 [Yad. Fiz. 28 (1978) 1597] [SPIRES].

[5] A.H. Mueller and H. Navelet, An inclusive minijet cross-section and the bare Pomeron in QCD, Nucl. Phys. B 282 (1987) 727 [SPIRES].

[6] D0 collaboration, B. Abbott et al., Probing BFKL dynamics in the dijet cross section at large rapidity intervals in $p \bar{p}$ collisions at $\sqrt{s}=1800 \mathrm{GeV}$ and $630 \mathrm{GeV}$, Phys. Rev. Lett. 84 (2000) 5722 [hep-ex/9912032] [SPIRES]. 
[7] V. Del Duca and C.R. Schmidt, Dijet production at large rapidity intervals, Phys. Rev. D 49 (1994) 4510 [hep-ph/9311290] [SPIRES].

[8] W.J. Stirling, Production of jet pairs at large relative rapidity in hadron hadron collisions as a probe of the perturbative Pomeron, Nucl. Phys. B 423 (1994) 56 [hep-ph/9401266] [SPIRES].

[9] L.H. Orr and W.J. Stirling, Dijet production at hadron hadron colliders in the BFKL approach, Phys. Rev. D 56 (1997) 5875 [hep-ph/9706529] [SPIRES].

[10] J. Kwiecinski, A.D. Martin, L. Motyka and J. Outhwaite, Azimuthal decorrelation of forward and backward jets at the Tevatron, Phys. Lett. B 514 (2001) 355 [hep-ph/0105039] [SPIRES].

[11] A. Sabio Vera and F. Schwennsen, The azimuthal decorrelation of jets widely separated in rapidity as a test of the BFKL kernel, Nucl. Phys. B 776 (2007) 170 [hep-ph/0702158] [SPIRES].

[12] C. Marquet and C. Royon, Azimuthal decorrelation of Mueller-Navelet jets at the Tevatron and the LHC, Phys. Rev. D 79 (2009) 034028 [arXiv:0704.3409] [SPIRES].

[13] J. Bartels, D. Colferai and G.P. Vacca, The NLO jet vertex for Mueller-Navelet and forward jets: the quark part, Eur. Phys. J. C 24 (2002) 83 [hep-ph/0112283] [SPIRES].

[14] J. Bartels, D. Colferai and G.P. Vacca, The NLO jet vertex for Mueller-Navelet and forward jets: the gluon part, Eur. Phys. J. C 29 (2003) 235 [hep-ph/0206290] [SPIRES].

[15] CMS collaboration, S. Cerci and D. d'Enterria, Low-x QCD studies with forward jets in proton-proton collisions at $\sqrt{s}=14 \mathrm{TeV}$ in CMS, AIP Conf. Proc. 1105 (2009) 28 [arXiv:0812.2665] [SPIRES].

[16] A.D. Martin, W.J. Stirling, R.S. Thorne and G. Watt, Parton distributions for the LHC, Eur. Phys. J. C 63 (2009) 189 [arXiv:0901.0002] [SPIRES].

[17] S.D. Ellis, Z. Kunszt and D.E. Soper, The one jet inclusive cross-section at order $\alpha_{s}^{3} .1$. Gluons only, Phys. Rev. D 40 (1989) 2188 [SPIRES].

[18] V.S. Fadin, BFKL news, hep-ph/9807528 [SPIRES].

[19] V.S. Fadin and A.D. Martin, Infrared safety of impact factors for colorless particle interactions, Phys. Rev. D 60 (1999) 114008 [hep-ph/9904505] [SPIRES].

[20] V.S. Fadin and L.N. Lipatov, BFKL Pomeron in the next-to-leading approximation, Phys. Lett. B 429 (1998) 127 [hep-ph/9802290] [SPIRES].

[21] M. Ciafaloni and G. Camici, Energy scale(s) and next-to-leading BFKL equation, Phys. Lett. B 430 (1998) 349 [hep-ph/9803389] [SPIRES].

[22] A.V. Kotikov and L.N. Lipatov, NLO corrections to the BFKL equation in $Q C D$ and in supersymmetric gauge theories, Nucl. Phys. B 582 (2000) 19 [hep-ph/0004008] [SPIRES].

[23] D.Y. Ivanov and A. Papa, Electroproduction of two light vector mesons in the next-to-leading approximation, Nucl. Phys. B 732 (2006) 183 [hep-ph/0508162] [SPIRES].

[24] A. Sabio Vera, The effect of NLO conformal spins in azimuthal angle decorrelation of jet pairs, Nucl. Phys. B 746 (2006) 1 [hep-ph/0602250] [SPIRES].

[25] F. Schwennsen, Phenomenology of jet physics in the BFKL formalism at NLO, DESY-THESIS-2007-001, Germany (2007) [hep-ph/0703198] [SPIRES]. 
[26] V.N. Gribov and L.N. Lipatov, Deep inelastic ep scattering in perturbation theory, Sov. J. Nucl. Phys. 15 (1972) 438 [Yad. Fiz. 15 (1972) 781] [SPIRES].

[27] L.N. Lipatov, The parton model and perturbation theory, Sov. J. Nucl. Phys. 20 (1975) 94 [Yad. Fiz. 20 (1974) 181] [SPIRES].

[28] G. Altarelli and G. Parisi, Asymptotic freedom in parton language, Nucl. Phys. B 126 (1977) 298 [SPIRES].

[29] Y.L. Dokshitzer, Calculation of the structure functions for deep inelastic scattering and $e^{+} e^{-}$ annihilation by perturbation theory in quantum chromodynamics (in Russian), Sov. Phys. JETP 46 (1977) 641 [Zh. Eksp. Teor. Fiz. 73 (1977) 1216] [SPIRES].

[30] G.P. Salam, A resummation of large sub-leading corrections at small x, JHEP 07 (1998) 019 [hep-ph/9806482] [SPIRES].

[31] M. Ciafaloni and D. Colferai, The BFKL equation at next-to-leading level and beyond, Phys. Lett. B 452 (1999) 372 [hep-ph/9812366] [SPIRES].

[32] M. Ciafaloni, D. Colferai and G.P. Salam, Renormalization group improved small-x equation, Phys. Rev. D 60 (1999) 114036 [hep-ph/9905566] [SPIRES].

[33] M. Ciafaloni, D. Colferai, G.P. Salam and A.M. Stasto, Renormalisation group improved small-x Green's function, Phys. Rev. D 68 (2003) 114003 [hep-ph/0307188] [SPIRES].

[34] R. Enberg, B. Pire, L. Szymanowski and S. Wallon, BFKL resummation effects in $\gamma^{*} \gamma^{*} \rightarrow \rho \rho$, Eur. Phys. J. C 45 (2006) 759 [Erratum ibid. C 51 (2007) 1015] [hep-ph/0508134] [SPIRES].

[35] J.R. Andersen, V. Del Duca, S. Frixione, C.R. Schmidt and W.J. Stirling, Mueller-Navelet jets at hadron colliders, JHEP 02 (2001) 007 [hep-ph/0101180] [SPIRES].

[36] S. Frixione and G. Ridolfi, Jet photoproduction at HERA, Nucl. Phys. B 507 (1997) 315 [hep-ph/9707345] [SPIRES].

[37] T. Hahn, CUBA: a library for multidimensional numerical integration, Comput. Phys. Commun. 168 (2005) 78 [hep-ph/0404043] [SPIRES].

[38] T. Sjöstrand, S. Mrenna and P.Z. Skands, PYTHIA 6.4 physics and manual, JHEP 05 (2006) 026 [hep-ph/0603175] [SPIRES].

[39] G. Marchesini et al., HERWIG: a Monte Carlo event generator for simulating hadron emission reactions with interfering gluons. Version 5.1 - april 1991, Comput. Phys. Commun. 67 (1992) 465 [SPIRES].

[40] D.Y. Ivanov and A. Papa, Electroproduction of two light vector mesons in next-to-leading BFKL: study of systematic effects, Eur. Phys. J. C 49 (2007) 947 [hep-ph/0610042] [SPIRES].

[41] F. Caporale, A. Papa and A. Sabio Vera, Collinear improvement of the BFKL kernel in the electroproduction of two light vector mesons, Eur. Phys. J. C 53 (2008) 525 [arXiv: 0707.4100] [SPIRES].

[42] M. Fontannaz, Dijet azimuthal correlations in hadron-hadron collisions at high energy, LPT-Orsay/09-86, France (2009).

[43] P. Aurenche, R. Basu and M. Fontannaz, Jet-jet and hadron-jet correlations in hadro- and electro-production, Eur. Phys. J. C 57 (2008) 681 [arXiv:0807.2133] [SPIRES]. 
[44] M. Fontannaz, J.P. Guillet and G. Heinrich, Is a large intrinsic $k_{T}$ needed to describe photon + jet photoproduction at HERA?, Eur. Phys. J. C 22 (2001) 303 [hep-ph/0107262] [SPIRES].

[45] P.M. Stevenson, Resolution of the renormalization scheme ambiguity in perturbative QCD, Phys. Lett. B 100 (1981) 61 [SPIRES].

[46] P.M. Stevenson, Optimized perturbation theory, Phys. Rev. D 23 (1981) 2916 [SPIRES]. 